\title{
Comparison of magnetization transfer methods for assessing macromolecular bound water in the brain and cervical spinal cord
}

Citation for published version (APA):

Lema Dopico, A. (2017). Comparison of magnetization transfer methods for assessing macromolecular bound water in the brain and cervical spinal cord. [Doctoral Thesis, Maastricht University]. Maastricht University. https://doi.org/10.26481/dis.20170626ald

Document status and date:

Published: 01/01/2017

DOI:

10.26481/dis.20170626ald

Document Version:

Publisher's PDF, also known as Version of record

Please check the document version of this publication:

- A submitted manuscript is the version of the article upon submission and before peer-review. There can be important differences between the submitted version and the official published version of record.

People interested in the research are advised to contact the author for the final version of the publication, or visit the DOI to the publisher's website.

- The final author version and the galley proof are versions of the publication after peer review.

- The final published version features the final layout of the paper including the volume, issue and page numbers.

Link to publication

\footnotetext{
General rights rights.

- You may freely distribute the URL identifying the publication in the public portal. please follow below link for the End User Agreement:

www.umlib.nl/taverne-license

Take down policy

If you believe that this document breaches copyright please contact us at:

repository@maastrichtuniversity.nl

providing details and we will investigate your claim.
}

Copyright and moral rights for the publications made accessible in the public portal are retained by the authors and/or other copyright owners and it is a condition of accessing publications that users recognise and abide by the legal requirements associated with these

- Users may download and print one copy of any publication from the public portal for the purpose of private study or research.

- You may not further distribute the material or use it for any profit-making activity or commercial gain

If the publication is distributed under the terms of Article $25 \mathrm{fa}$ of the Dutch Copyright Act, indicated by the "Taverne" license above, 
Comparison of magnetization transfer methods for assessing macromolecular bound water in the brain and cervical spinal cord

\author{
DISSERTATION \\ to obtain the degree of Doctor at Maastricht University, \\ on the authority of the Rector Magnificus, Prof. dr. Rianne M. Letschert \\ in accordance with the decision of the Board of Deans, \\ to be defended in public \\ on Monday $26^{\text {th }}$ of June 2017, at 14:00 hours
}

by

\title{
Alfonso Lema Dopico
}


Supervisor

Prof. dr. P. M. Matthews, Imperial College of London, United Kingdom

Co-Supervisor

Dr. R. D. Newbould, Imanova Ltd, London, United Kingdom

\section{Members of the Degree Committee}

Prof. dr. E. Formissano, chair, Maastricht University, Netherlands

Prof. dr. R. Heeren, Maastricht University, Netherlands

Prof. dr. P. Piccini, Imperial College of London, United Kingdom

Dr. P. Muraro, Imperial College of London, United Kingdom 


\section{Institutions}

GlaxoSmithKline plc, United Kingdom

Imanova Ltd, United Kingdom

Maastricht University, Netherlands

Marie Skłodowska-Curie Actions Innovative Training Networks 


\section{Abstract}

Magnetization transfer (MT) imaging is a magnetic resonance imaging technique that generates contrast dependent upon the phenomenon of magnetization exchange between semi-solid macromolecular protons and water protons. This technique has the ability to indirectly image semi-solids, such as protein matrices and cell membranes, whose magnetization dies away too quickly to be imaged directly based on relaxation properties of water interacting with the semisolid lipid protons. Preliminary results suggest that MT quantification may allow improved characterization of the pathologically heterogeneous lesions of multiple sclerosis by providing a measure of demyelination through a sensitivity to myelin proteins. However, MT imaging, as currently most commonly applied, is only a semi-quantitative technique that reflects mixed tissue and experimental parameters in addition to MT.

In this thesis a novel quantitative MT imaging technique (MTsat) is applied in both brain and cervical spinal cord in order to test whether addressing this limitation can enhance discrimination of neuropathology in people with multiple sclerosis (MS). It was found that MTsat can discriminate between pathology in both brain and cervical spinal cord that is associated with different patient disability levels. Also, signal from regions of the brain where conventional MRI does not delineate tissue damage shows significant correlation with disability scores. 


\section{Abstract}

Magnetisatie transfer (MT) beeldvorming is een magnetische resonantie beeldvormingstechniek dat contrastafhankelijk genereert op het fenomeen magnetisatie uitwisseling tussen semi solide macromoleculaire protonen en waterprotonen. Deze techniek biedt de mogelijkheid om indirect afgebeelde semisolide deeltjes, zoals matrixproteïnen en celmembranen, waarvan de magnetisatie te snel afneemt, direct af te beelden, gebaseerd op wisselwerking van de ontspanningseigenschappen van water met de semi-solide lipide protonen. Voorlopige resultaten suggereren dat MT kwantificatie mogelijk verbeterde karaktereigenschappen van de pathologische heterogene laesies van multiple sclerosis erkent door een hoeveelheid demyelinisatie te geven door middel van een gevoeligheid voor myeline proteïne. Echter MT beeldvorming, zoals het op dit moment voornamelijk wordt toegepast, is slechts een semi-kwantitatieve techniek dat naast MT ook gemengde weefsel- en experimentele parameters weerspiegelt.

In dit proefschrift wordt een ongekende kwantitatieve MT beeldvormingstechniek (MTsat) toegepast in zowel het brein alsmede de cervicale ruggenmerg om te testen of het behandelen van deze beperking het onderscheid van neuropathologie bij mensen met multiple sclerosis (MS) verbetert. Er is aangetoond dat MTsat wel degelijk onderscheid kan maken tussen de pathologie in zowel het brein als het cervicale ruggenmerg dat wordt geassocieerd met de verschillende invaliditeitsniveaus van patiënten. Tevens, een signaal uit delen van het brein, waar conventionele MRI geen weefselbeschadiging laat zien, toont significante correlatie met de invaliditeitsscores. 


\section{Resumen}

La imagen por transferencia de magnetización (MT) es una técnica de imagen por resonancia magnética nuclear que genera contraste teniendo como base el fenómeno del intercambio de magnetización entre protones de macromoléculas de semisólidos y agua. Esta técnica tiene la propiedad de poder generar imágenes de semisólidos indirectamente, como pueden ser las matrices de proteínas y las membranas celulares, cuya magnetización cae demasiado rápido como para producir imágenes directamente basándose en las propiedades de relajación de los protones del agua interaccionando con los de lípidos. Resultados preliminares sugieren que la cuantificación de MT podría permitir una mejora en la caracterización de las lesiones patológicamente heterogéneas en esclerosis múltiple ofreciendo una medida de la desmielinizacion a través de las proteínas de la mielina. Sin embargo, la imagen MT, en su más amplio rango de aplicación, es solamente una técnica semicuantitativa que refleja una mezcla de tejido y parámetros experimentales junto con MT.

En esta tesis, una nueva técnica cuantitativa de imagen (MTsat) se aplica en el cerebro y la espina dorsal cervical con el fin de evaluar si el control de esta limitación puede mejorar la discriminación neuropatológica en sujetos con esclerosis múltiple (MS). Se encontró que MTsat puede discriminar la patología en el cerebro y la espina dorsal cervical que está asociada con los diferentes grados de incapacidad de los pacientes. Además, la señal de regiones del cerebro en donde las técnicas de imagen convencionales no muestran daño de tejido arroja una correlación significativa con las escalas de incapacidad. 


\section{Résumé}

L'imagerie de transfert d'aimantation (TA) est une technique d'imagerie par résonnance magnétique permettant de générer des contrastes résultant du phénomène d'échange d'aimantation entre protons macromoléculaires semi-solides et protons d'eau. Cette technique permet de visualiser indirectement les semi-solides comme les matrices protéiques et les membranes cellulaires, dont l'aimantation ne dure pas assez longtemps pour une imagerie reposant directement sur les propriétés de relaxation d'une eau interagissant avec des protons de lipides semi-solides. Les premiers résultats suggèrent que la quantification $\mathrm{TA}$ pourrait permettre d'améliorer la caractérisation de lésions pathologiquement hétérogènes dues à une sclérose en plaques, en fournissant une mesure de la démyélinisation grâce à la sensibilité des protéines de myéline. Néanmoins, l'imagerie TA telle qu'elle est aujourd'hui le plus communément utilisée n'est qu'une technique semi-quantitative qui reflète en plus du transfert d'aimantation les paramètres de divers tissus et expérimentaux.

Dans cette thèse, une nouvelle technique d'imagerie TA quantitative (ATsat) est appliquée au cerveau et à la moelle épinière, dans le but de savoir si apporter une réponse à cette limitation permettrait d'améliorer la discrimination des neuropathologies auprès de patients atteints de sclérose en plaques. Il a été constaté que l'imagerie ATsat permet de discriminer les pathologies, présentes au niveau du cerveau comme de la moelle épinière, associées à différentes échelles d'incapacité chez les patients. De plus, un signal en provenance de zones du cerveau où une IRM conventionnelle ne permet pas de délimiter les lésions tissulaires indique une corrélation significative avec les échelles d'incapacité. 


\section{Acknowledgments}

I am grateful to many people for the support and assistance that has made this thesis possible: all the researchers and technicians who were involved in acquiring the MRI data and the MS patients; Rexford Newbould, from Imanova; Phillip Murphy, from GSK; and the Maastricht University promoters of the project, Professor Peter de Weerd and Ermo Daniels. I would like to express my appreciation for the guidance and insight that my supervisor, Professor Paul Matthews, has provided at every stage of this work. Lastly, I would like to thank to my family, for all the support and encouragement throughout.

In addition, I would like to acknowledge the financial support I have received from the Marie Curie Research Programme, for which I am grateful. 


\section{Contents}

$\begin{array}{ll}\text { Abstract (English) } & \text { i }\end{array}$

Abstract (Dutch) ii

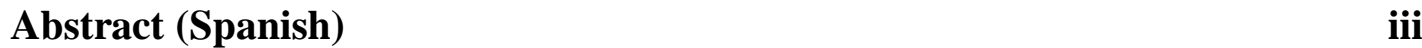

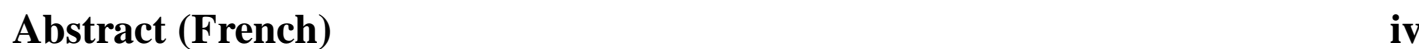

Acknowledgments $\quad$ v

List of figures $\quad x$

List of tables

1. Introduction 1

1.1 Aim and objectives 3

1.2 Outline of the thesis 4

2. Multiple sclerosis 6

$\begin{array}{ll}2.1 \text { Introduction } & 6\end{array}$

2.2 Symptoms $\quad 8$

2.3 Classification different types of MS 9

2.3.1 Relapsing-remitting multiple sclerosis (RRMS) 9 
2.3.2 Secondary-progressive multiple sclerosis (SPMS)

2.3.3 Primary-progressive multiple sclerosis (PPMS)

2.3.4 Progressive-relapsing multiple sclerosis

2.4 Diagnosis

2.4.1 Magnetic resonance imaging

2.4.2 Cerebrospinal fluid

2.4.3 Evoked potentials

2.5 Causes

2.6 White matter pathology

2.7 Grey matter pathology

2.8 Measuring disability

2.8.1 EDSS

2.8.2 T25FW

\section{Magnetic resonance imaging}

3.1 Introduction

3.2 Basic principles

3.2.1 RF excitation and relaxation

3.3 Imaging principles

3.4 MRI sequences

3.4.1 Spin echo sequence

3.4.2 Gradient echo sequence

3.4.3 Inversion recovery sequence 
4.1 Introduction

4.2 Magnetization transfer physics: relaxation mechanisms

4.2.1 Cross relaxation: dipole-dipole interactions

4.2.2 Saturating pulses

4.3 Magnetization transfer ratio: MTR

4.4 Magnetization transfer saturation: MTsat

4.5 Magnetization transfer imaging and disability

5. Comparison of MTR and MTsat

5.1 Introduction

5.2 Methods

5.3 Results

5.4 Discussion

5.5 Conclusion

6.1 Introduction

6.2 Methods

6.3 Results

6.4 Discussion

6.5 Conclusion 
7. Relationships between microstructure of the brain and cervical cord across different levels of disability in patients with multiple sclerosis: atrophy and $\begin{array}{ll}\text { MT histogram analyses } & 100\end{array}$

$\begin{array}{ll}7.1 \text { Introduction } & 100\end{array}$

$\begin{array}{ll}\text { 7.2 Methods } & 102\end{array}$

$\begin{array}{ll}7.3 \text { Results } & 105\end{array}$

$\begin{array}{ll}\text { 7.4 Discussion } & 106\end{array}$

$\begin{array}{ll}7.5 \text { Conclusion } & 108\end{array}$

$\begin{array}{lr}\text { 8. Conclusions } & 117\end{array}$

$\begin{array}{ll}\text { 8.1 Achieving the objectives } & 117\end{array}$

$\begin{array}{lr}\text { 8.2 Future challenges } & 119\end{array}$

$\begin{array}{ll}\text { Appendix A: Binary Spin Bath model and MTsat } & 121\end{array}$

$\begin{array}{ll}\text { Appendix B: Peer-Reviewed Publication } & 127\end{array}$

$\begin{array}{ll}\text { Appendix C: Valorisation } & 133\end{array}$

$\begin{array}{ll}\text { Bibliography } & 138\end{array}$ 


\section{List of figures}

1.1 Atlas of MS 2013

1.2 Conventional MRI images in brain and cervical spinal cord

2.1 Robert Carswell and Santiago Ramon y Cajal drawings

2.2 Clinical subtypes of MS

2.3 Demyelinated WM lesion

2.4 Remyelinated WM lesion

2.5 Correlation of MRI and histology in post-mortem

$3.1 \mathrm{cMRI}$ and FLAIR images

3.2 T1w and post-gadolinium T1 images

3.3 T2 FLAIR and T1w images

4.1 Spectral lines of the free and bound pools

4.2 Resonance pulses and bound pool

4.3 General excitation recovery sequence with pulses

4.4 MT FLASH and PDw/T1w reference images and MTsat map

4.5 Axial MT FLASH images at different flip angles 
4.8 MTsat and frequency offset

5.1 Average whole brain MTR and MTsat histograms 


\section{List of tables}

2.1 Neurologic disability classification

2.2 Multiple sclerosis functional composite

5.1 Summary of the clinical characteristics of the brain study

5.3 Spearman correlations: brain metrics and EDSS

5.4 Spearman partial correlations: brain metrics with EDSS

5.5 Spearman correlations: brain metrics with $\mathrm{T} 25 \mathrm{FW}$

6.1 Summary of the clinical characteristics of the cervical cord study

6.3 Spearman correlations: cervical cord with EDSS

6.4 Spearman partial correlations: cervical cord with EDSS

6.5 Spearman correlations: cervical cord with T25FW 
7.4 Two multivariate regressions with cord MTR metrics

7.5 Two multivariate regressions with cord MTsat metrics 


\section{Chapter 1}

\section{Introduction}

Multiple sclerosis is an autoimmune disease of the central nervous system. It causes neurological disability resulting in part from the interruption of myelinated tracts. Currently, there are more than 100,000 people in the UK and more than 2 million people throughout the world (Figure 1.1) with this disease. It is the most common disabling illness of young adults in the UK and it is twice as common in women as in men. The diagnosis made by the neurologists is mainly based on the so called McDonald's criteria ${ }^{1}$ which allows diagnosis of multiple sclerosis on the basis of clinical findings and supporting evidence from tests, such as magnetic resonance imaging of the brain and spinal cord. Clinical performance scales are used to rate disability, like the Kurtzke's Expanded Disability Status $\mathrm{Scale}^{2}$ and the Multiple Sclerosis Functional Composite ${ }^{3}$, an important functional component of which is the Timed 25-Foot Walk scale.

Magnetic resonance imaging is used not only to assess the diagnosis, but also to monitor the patients during their life. Conventional imaging has limitations in evaluation of the causes of disability and lacks pathological specificity (Figure 1.2). New imaging techniques can overcome these limitations. Among them, magnetization 
transfer generates contrast dependent on the phenomenon of magnetization exchange between macromolecular and free water protons. It can give quantitative information on the nature of pathological changes and add specificity to diagnostic images, enhancing assessment of lesion burden and the degree of disability.

PREVALENCE BY COUNTRY (2013)

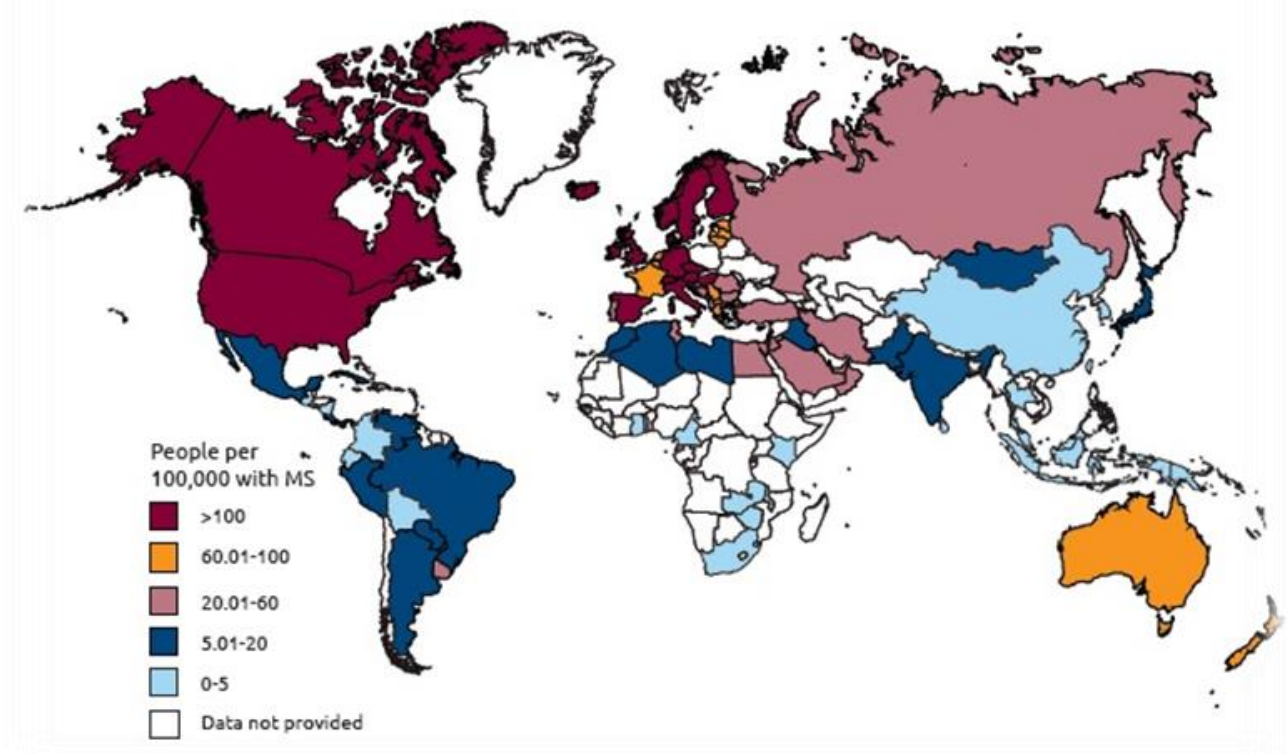

Figure 1.1: Atlas of MS 2013. Mapping multiple sclerosis around the world. (C) Multiple Sclerosis International Federation. 


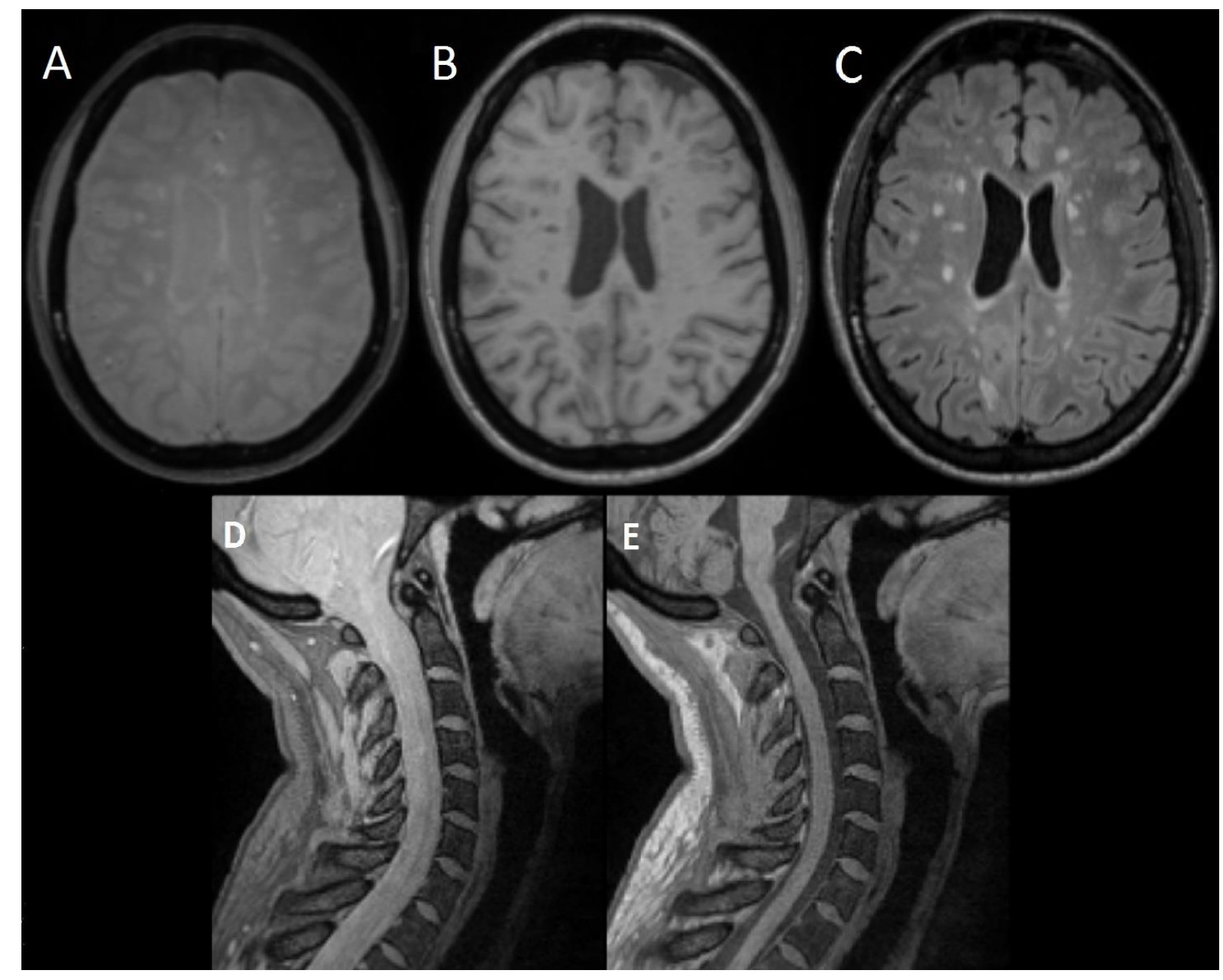

Figure 1.2: Conventional MRI images in the brain and cervical spinal cord of a multiple sclerosis patient. PD-weighted (A), T1-weighted (B) and T2-weighted (C) in the brain. PD-weighted (D) and T1-weighted (E) in the spinal cord. Patient Research Cohort-Rapidly Evolving Multiple Sclerosis (PRC-REMS) Imperial College London.

\subsection{Aim and Objectives}

The main aim of the work presented in this thesis is to assess the relative capability of two magnetization transfer imaging techniques to discriminate between disability levels in multiple sclerosis. 
The first goal is to have an extant cohort of MS patients which can be representative of the target phenotype for clinical studies. Scanning and disability tests will be made following established protocols. Then, after the acquisition of the images, they are going to be debugged of artefacts and imprecisions at the time of the scanning. Lesions have to be demarked in both brain and cervical spinal cord and MT maps made. Finally, the data analysis has to be implemented.

The second goal is to compare both MT techniques across different regions of interest of the brain and in the cervical cord, which is a major site of pathology but more challenging in discrimination of relevant pathology.

\subsection{Outline of the Thesis}

Chapters 2, 3 and 4 constitute an introduction. Chapter 2 describes multiple sclerosis, characterising the disease, its most common symptoms and diagnostic techniques, and briefly discussing recent theories about its causes. The final section of this chapter is dedicated to introduce the clinical scales which are used to monitor the disability.

In Chapter 3 the basic principles of magnetic resonance imaging are described in order to better understand the limitations of the conventional techniques and their lack of pathological specificity of the lesions and the tissue damaged, and also their modest correlation with the degree of disability in people with MS.

Chapter 4 deals with the magnetization transfer imaging and, after describing the model, which explains the magnetization exchange between free water protons and molecule water protons, the techniques which will be applied in this thesis are introduced. Firstly, magnetization transfer ratio (MTR) is defined and it is used as the standard references in terms of conventional magnetization transfer. Then, the novel 
quantitative magnetization transfer saturation technique (MTsat), which has been developed recently and overcomes some of the limitations of MTR, is explained. Due to its potential improvements over the standard MTR, it could provide more sensitive parameters for assessment of changes in magnetization transfer in multiple sclerosis.

Chapters 5, 6 and 7 are dedicated to descriptions of research outcomes. Chapter 5 is focused on the brain. It describes study of a cohort of 121 patients in which the magnetization transfer maps are made using standard MTR and MTsat and compared. Different regions of interest are studied using histogram analysis versus disability, highlighting differences in results between those magnetization techniques.

Chapter 6 is focused on the cervical cord. It is based on a study of 52 patients. Like in the brain study, the magnetization transfer maps again are used to assess the capabilities of the two methods to discriminate levels of disability.

Destructive processes in multiple sclerosis that affect the brain versus the spinal cord lead to tissue loss. In Chapter 7, an analysis atrophy of brain and spinal cord relates these measures to the metrics from the magnetization transfer maps in order to estimate the relative contributions from different aspects of the pathologies. This reports results from study of a cohort of 43 patients.

Finally, a brief discussion on the whole thesis will be contained in Chapter 8 . 


\section{Chapter 2}

\section{Multiple sclerosis}

\subsection{Introduction}

Multiple sclerosis (MS) is an autoimmune demyelinating disease. It represents a common cause of neurological disability in young adults resulting from the interruption of myelinated tracts in the central nervous system. The first attack often occurs when the patient is between the ages of 20 and 40, although this and subsequent attacks may not be recognized as MS until later.

The first description of MS dates back to the $14^{\text {th }}$ century ${ }^{4}$, but it was the neurologist Jean Martin Charcot who first described anatomically methods the correlations between the clinical features of MS and pathological post-mortem changes $^{5,6}$. The recognition of MS as a distinct disease was quite a challenge for the time. As many diseases in the early $19^{\text {th }}$ century that would be categorized as either neurological or psychiatric would have been grouped into a general class of nervous disorders with no separation between individual conditions. Such an attempt at the classification of neurological diseases had not made before Charcot. He detailed the description of MS in 1868 and he described it as "la sclérose en plaques", accompanied by the first drawings illustrating the expressions of lesions from the 
ventricles into the cerebral hemispheres, and provided the earliest insight into the pathology of MS involving both the brain and spinal $\operatorname{cord}^{5}$. He would go on to describe the various forms of MS such as cephalic, spinal and mixed cerebrospinal, correlating symptoms with post-mortem findings ${ }^{6}$. In addition he was the first person to diagnose MS on a living patient. The name "multiple sclerosis", therefore, means that a patient has more than one sclerosis, which is a plaque, or lesion that can appear in the white matter or in the grey matter of the brain and/or the spinal cord.

The discovery of myelinated nerve fibres is credited by Santiago Ramon y Cajal to Christian G. Ehrenberg who in 1833 described nerve fibres as consisting of cylindrical tubes of between 4 and $10 \mu \mathrm{m}$ in diameter. In each he distinguished "four parallel lines, two of which form the external boundary lines, while the inner indicate the boundaries of the interior cavity" . Cajal recognized in 1909 that the axis cylinder is the axonal process and that myelin sheath is external and a protective layer surrounding the axons. Lesions can lead to a breakdown of the myelin sheath, which is known as demyelination. When the myelin sheath is lost (or thins substantially), the axon is not able to function optimally, as saltatory conduction cannot occur ${ }^{8-10}$. Although the myelin can repair itself (a process that is called remyelination ${ }^{11}$ ), this is incomplete in multifocal lesions of MS. In some cases, the inflammatory response in MS leads to transection of axons and total interruption of electrical conduction. 

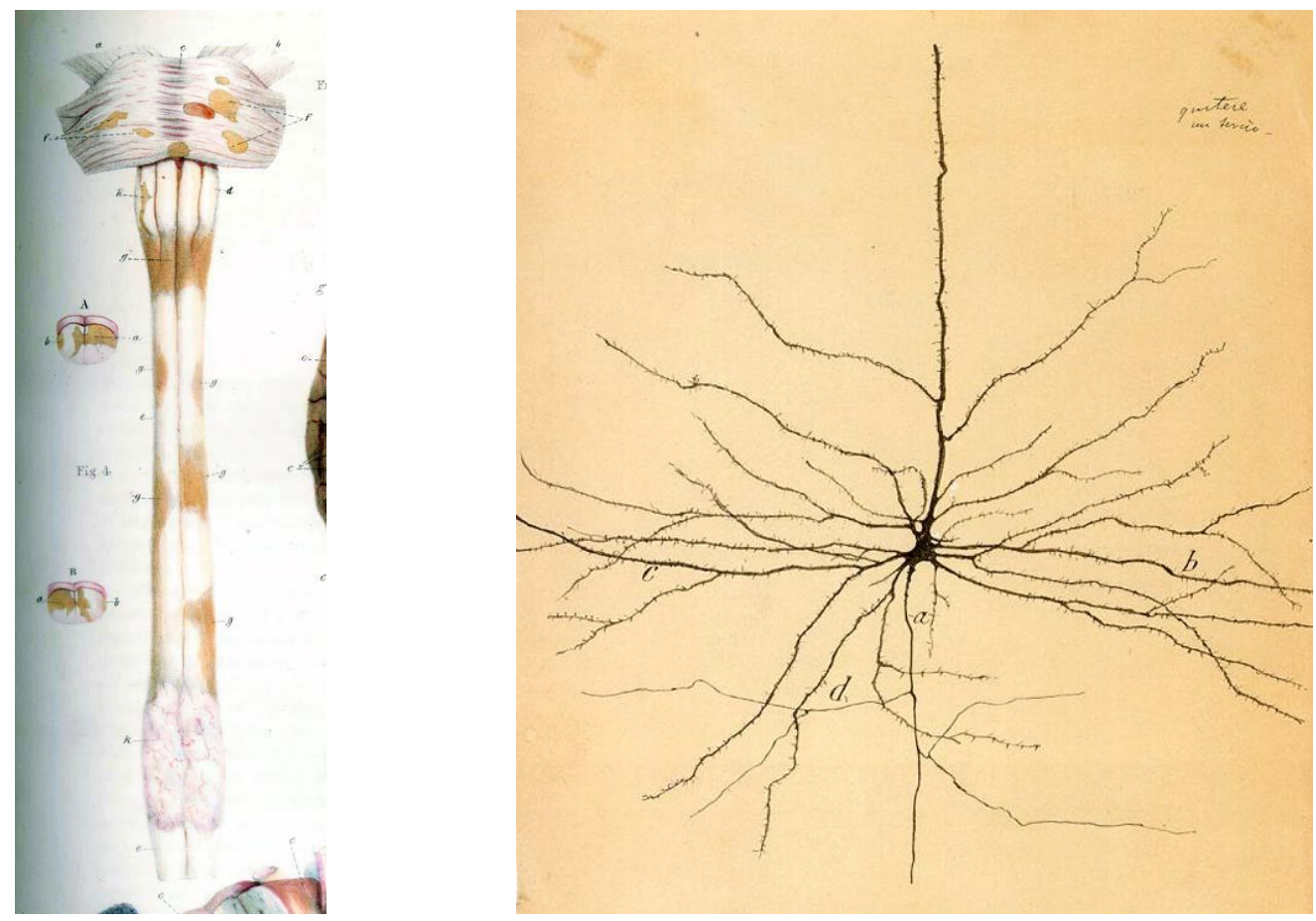

Figure 2.1: On the left, Robert Carswell was the first to portray the plaques of multiple sclerosis, although he did not identify them as such, 1838 () Glasgow University. On the right, drawing of a pyramidal neuron from a Golgi's stained tissue by Santiago Ramon y Cajal, 1899 (C) Cajal Institute.

\subsection{Symptoms}

The symptoms, severity, and course of MS vary widely depending partly on the sites of the plaques and the extent of the demyelination. The specific symptoms that appear depend upon both which part of the central nervous system is affected and the function of the damaged nerve. This is the reason why MS is an extremely unpredictable disease. Lesions can occur anywhere in the central nervous system and the stages and rates of demyelination vary depending on them. The first experience of the condition is described as the clinically isolated syndrome (CIS), which typically occurs between the ages of 20 and 40 . 
There are many symptoms encountered in MS. They may be temporary and mild, or more persistent and troublesome. Early symptoms may include: loss of balance; lack of coordination; thinking problems; blurred or double vision; numbness; tingling and weakness in an arm or leg. In addition to the persistence of early symptoms, some patients experience the following symptoms as the disease progresses: tremors; facial pain; hearing loss; spasms; speech difficulties; problems in the gastrointestinal, urinary and genital tracts and problems in concentration and memory.

\subsection{Classification of different types of MS}

Patients usually experience a clinically isolated syndrome (CIS) -a first neurologic event suggestive of MS- for at least 24 hours, with the symptoms and signs indicating either a single lesion (monofocal) or more than one lesion (multifocal) within the CNS. After presentation, four courses of progression of MS can ensue.

\subsubsection{Relapsing remitting multiple sclerosis (RRMS)}

This is the most frequent presentation for younger people and is the most common form of MS. Patients with RRMS have attacks of neurological dysfunction lasting days to weeks followed by complete to near complete recovery. This accounts for about $85 \%$ of patients with MS. After approximately 10-20 years, the majority of patients with RRMS have entered into another stage of the disease called secondary progressive MS, where there is gradual accumulation of neurological dysfunction without recovery (see 2.3.2). Diagnosis of RRMS could be either on two relapses 
separated by more than 30 days or one relapse and a magnetic resonance imaging (MRI) scan three months later, showing new lesions.

\subsubsection{Secondary progressive multiple sclerosis (SPMS)}

Most people with RRMS will eventually develop SPMS, which is characterised by a worsening of disability in the absence of episodic relapses. Around $20 \%$ of patients are in an SPMS stage, typically reached after the age of 45 .

Accumulation of demyelination and neuronal damage during RRMS triggers the subsequent progression of neurological disability during SPMS ${ }^{12}$. Therefore, primary inflammatory demyelination underlies early axonal loss during RRMS; however, this axonal loss is clinically silent. The transition from RRMS to SPMS has been suggested to occur when a threshold of axonal loss is reached, and the compensatory capacity of the CNS is surpassed, resulting in the subsequent development of permanent neurological symptoms. The level of inflammatory activity during RRMS determines the rate of neurodegeneration and the point at which a patient is said to have SPMS.

\subsubsection{Primary progressive multiple sclerosis (PPMS)}

This type is not very common, occurring in about $10 \%$ of patients. They experience a slow progression of the disease without clearly definable relapses. If there have been no previous relapses and if there is a progression of disability over at least one year, if there is an MRI scan that shows lesions consistent with MS, and if there is evidence of 
MS detected by examining the spinal fluid collected during a lumbar puncture, PPMS is diagnosed.

\subsubsection{Progressive relapsing multiple sclerosis}

This is a rare form of MS (5\%). Patients with this form of MS get progressively and steadily worse and also suffer relapses. Sometimes they recover briefly, but they never experience remission of the disease.

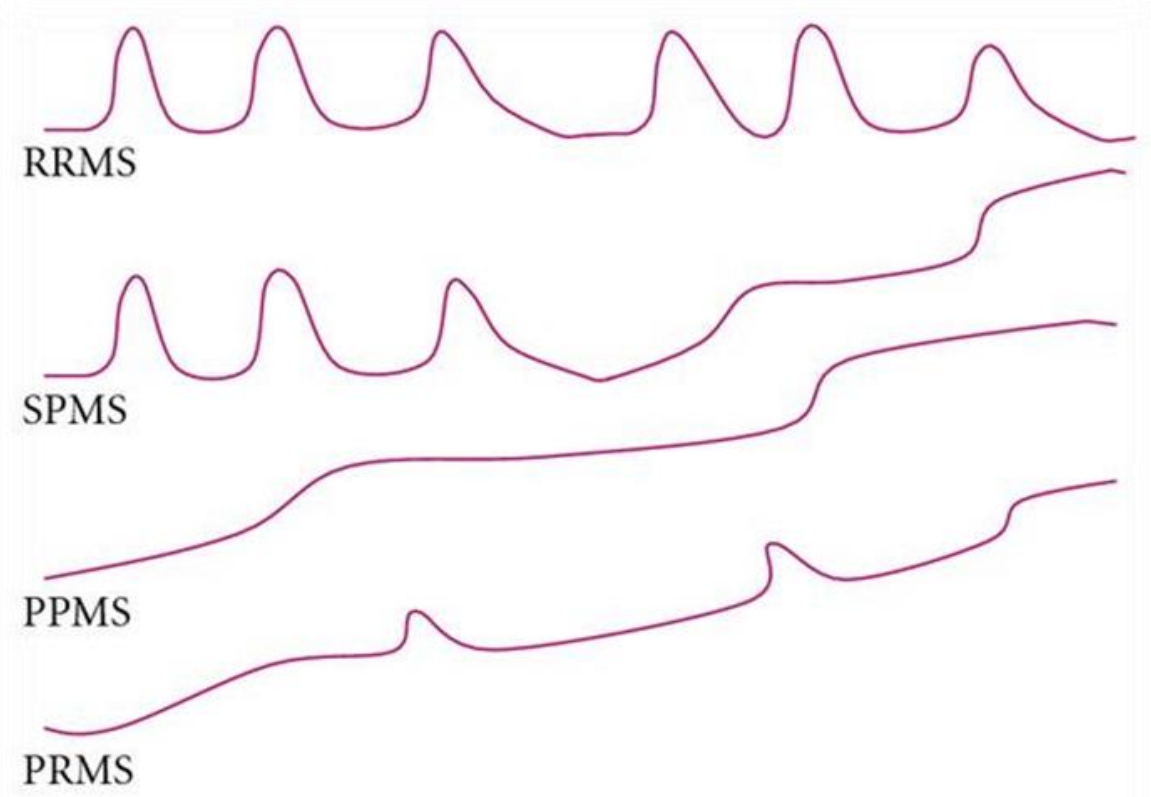

Clinical subtypes of MS

Figure 2.2: These are the four common clinical subtypes of MS. RRMS: a clinical attack (relapsing phase) follows by complete or partial recovery (remitting phase). SPMS: Symptoms are continuously and gradually worsening after a period of RRMS.

PPMS: Symptoms keep worsening without relapsing-remitting phases. Progressive 
relapsing MS: MS with characteristics of both PPMS and RRMS. (Hong-Liang Zhang et al. Clinical and Developmental Immunology 2010)

\subsection{Diagnosis}

There is no specific diagnosis test for MS. MS diagnosis is based on the objective clinical, imaging or laboratory evidence of lesions disseminated in space and time, and the exclusion of other possible diseases according to symptomatology. Clinical features like MRI exams, cerebrospinal fluid analysis and visual evoked potentials contribute to the diagnostic procedure.

In order to assess and predict future disability, a scale called the Expanded Disability Status Scale ${ }^{2}$ is commonly used both in clinical practice and for clinical trials. It is a composite scale comprising steps between 0 and 10 in 0.5 point increments, representing the rise of disability as the score increases. This scale has a wide acceptance and covers the full range of the disease from asymptomatic to death from MS.

Another scale is the Multiple Sclerosis Functional Composite ${ }^{13}$. It is the combination of the following tests: timed 25 foot walk, the nine-hole peg done with each hand and the paced auditory serial addition (Table 2.2). Test results are converted to $\mathrm{Z}$ scores, which allow the use of parametric statistics. The test was developed by a committee of the National MS Society to improve test reliability and objectivity in MS clinical trials. 


\subsubsection{Magnetic resonance imaging.}

Lesions seen in MRI may demonstrate MS criteria of dissemination in space and when MRI is repeated with at least 3 months interval, changes in lesion burden may also fulfil MS criteria of dissemination in time. Spinal cord MRI, which is abnormal in approximately $70 \%$ of patients, may provide additional findings consistent with MS criteria of dissemination in space.

\subsubsection{Cerebrospinal fluid.}

Oligoclonal bands arise from proteins called immunoglobulins in the cerebrospinal fluid (CSF). The presence of these proteins indicates inflammation of the CNS and adds to positive diagnostic criteria suggestive of MS. However, it is not a feature specific to MS, as they also can be detected in other inflammatory, infectious and tumoral diseases of the $\mathrm{CNS}^{14}$.

\subsubsection{Evoked potentials.}

They provide a non-invasive technique for analysing different neurological pathways which may impaired in MS and, as electrophysical functional measures, complement MRI. Visual evoked potentials may indicate electrical abnormalities of the optic nerve, which are suggestive of optic neuritis. Brainstem and sensory evoked potentials may reveal lesions of the auditory and the spinal cord sensory pathways, respectively ${ }^{15}$. 


\subsection{Causes}

The cause or causes of multiple sclerosis are still unknown. Genetic factors certainly play a role in $\mathrm{MS}^{16,17}$. No single gene, however, is likely to be responsible for causing $\mathrm{MS}^{18}$. Rather, the current theory is that the disease occurs in people with a genetic susceptibility who are exposed to some environmental assault (a virus or a toxin) that disrupts the blood-brain barrier. Immune factors converge in the nerve cells and trigger inflammation and an autoimmune attack on myelin and axons. Still, a number of disease patterns have been observed in patients, so that MS is often considered not as single disorder, but it may instead represent several diseases with different causes.

MS is not a genetically inherited disorder in a simple Mendelian fashion since no gene has been found to be specially impaired in MS patients, and the disease is not associated with a pattern monogenic transmission. However, it does show significant heritability, suggesting genetic factors. MS is a multifactorial disease, influenced by environmental interactions with any genetic factors ${ }^{19}$. The role of environmental factors has been supported by the observation that susceptibility to MS is higher in Nordic populations than in populations living near the Equator ${ }^{20}$. A genetic contribution has been suggested by the existence of multiplex families (those ones with at least two members suffering from MS, 10-15\%), the higher risk in first degree (20-40 fold higher) and the higher MS concordance rate in monozygotic twins (6$40 \%)$ than in dizygotic twins $(2.7-4.7 \%)$. There is no prenatal diagnostic test for MS.

Infectious organisms, most likely viruses that can affect the central nervous system, are suspected of triggering the autoimmune response in people genetically susceptible to MS. Although many infectious microorganisms have been investigated, 
no one organism has emerged as a proven trigger. It is possible that different patients may be affected by different organisms, and these infections cause some, but not all, cases of MS. At present, microorganisms like Epstein-Barr virus and Chlamydia Pneumoniae are under suspicion ${ }^{21-23}$.

\subsection{White matter pathology}

MRI allows the in vivo detection of white matter (WM) lesions, which sometimes correlate with neurological symptoms. However, any discussion of WM pathology (and, indeed, a thesis focused on its better elucidation) needs to acknowledge that MS patients also display neurological symptoms that cannot be explained by the identified WM pathology, but are more consistent with cerebral and cerebellar grey matter (GM) pathology ${ }^{24}$. The severity of MS clinical outcome thus does not only result from the extent of WM damage, but it rather represents a summation of consequences of WM and GM tissue damage, tissue repair and cortical reorganization ${ }^{25,26}$. Based on the distribution of the demyelinated areas, three general types of neocortical lesions were described $^{27}$ : Type I, which were demyelinated both WM and cortex; Type II, demyelinated lesions that were intracortical; and the cortical lesions extended into the cortex from the pial surface were defined as Type III.

Plaques represent that area where the myelin has been destroyed and axons have been relatively spared. Plaques seem to evolve from demyelination, a consequence of immune responses of the infiltrating lymphocytes and macrophages that define active plaques. The active plaque in white matter corresponds to the area where gadolinium enhancement can be seen on contrast enhanced MRI ${ }^{28}$. With demyelination and gliosis due to astrocyte proliferation occurs, lesions become 
apparent chronically on T2-weighted images. "Black holes" on T1-weighted images are a feature of irreversible necrosis. Remyelination can occur. This has structural differences, appearing thinner on microscopic observation ${ }^{29}$. Partial remyelination is thought to be the basis for the Uhthoff phenomenon, an increase in transmission block that occurs when the body temperature is raised and the basis for the heat-sensitivity of MS patients ${ }^{30,31}$. Plaques generally are present throughout the CNS in the white matter, but areas of predilection include the corpus callosum, the roof of the lateral ventricles, and the fourth ventricle. In the spinal cord, lesions occur most frequently in the posterior and lateral funiculi, mostly at the cervical level. Demyelination in the brainstem can be a cause of death with respiratory compromise. Immunologic studies of MS plaques have revealed that glial cells (both astrocytes and microglial cells) are activated $^{32}$.

One of the most debated issues in MS research has been the question of the degree of neuropathological abnormality of the so-called normal appearing white matter (NAWM) defined by conventional MRI. After segmentation of hyperintense lesions on T2-weightened images, NAWM is defined conventionally as the WM volume outside areas of abnormally high WM signal intensity. The macroscopic lesion load leading to hyperintense T2 lesions in an MS brain constitutes typically at most a few percent of the total WM volume. Thus, most of the changes should happen in the NAWM. Also, the definition of brain pathology that is responsible for functional impairment and disability demands the understanding of the ways in which neurons interact via axonal projections in the WM which includes both volumes of lesions and NAWM.

Several magnetic resonance measures of pathological change show abnormalities in the NAWM of people with MS such as: the progressive loss of 
volume $^{33}$, relaxation time measurements showing diffuse changes ${ }^{34}$, an abnormally low magnetization transfer ${ }^{35}$, abnormally increased diffusion anisotropy ${ }^{36}$ and abnormalities of magnetic resonance spectroscopy consistent with diffuse axonal injury ${ }^{37}$.
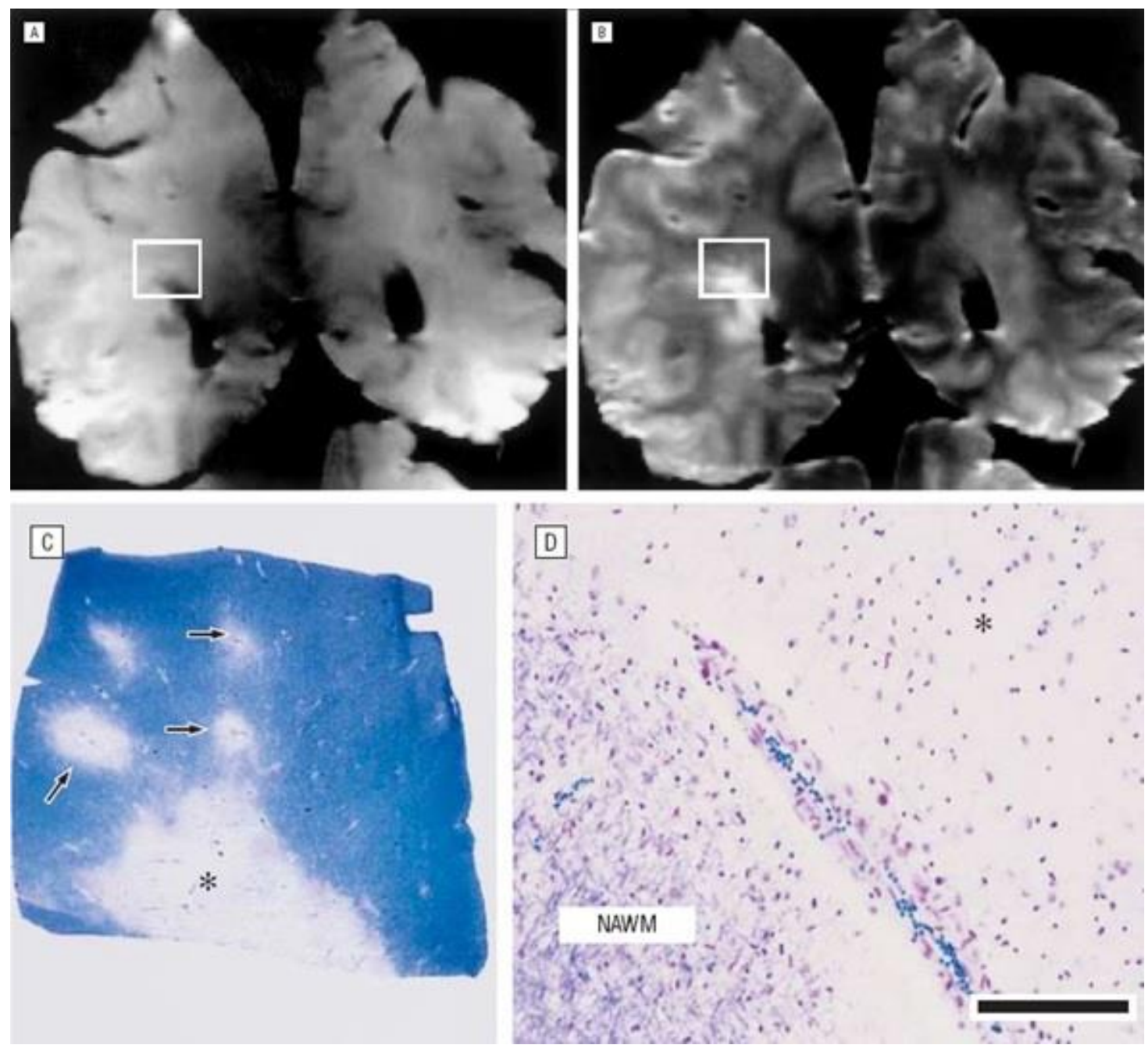

Figure 2.3: Demyelinated WM lesion. A and B, Coronal post-mortem T1-weighted (A) and T2-weighted (B) MRI. The frames show a T1 hypointense lesion that was dissected and further analysed histogically. C, Photograph of a Luxol fast blue (LFB) stained, paraffin-embedded brain tissue section matching the frame on the MRIs shows a large demyelinated lesion in the white matter (asterisk) and a few smaller 
ones (arrows). D, at higher power, the lesion is totally demyelinated (asterisk) compared with the adjacent NAWM (scale bar $=20 \mu \mathrm{m}$ ). (Barkhof $\mathrm{F}$ et al. Arch Neurol. 2003)
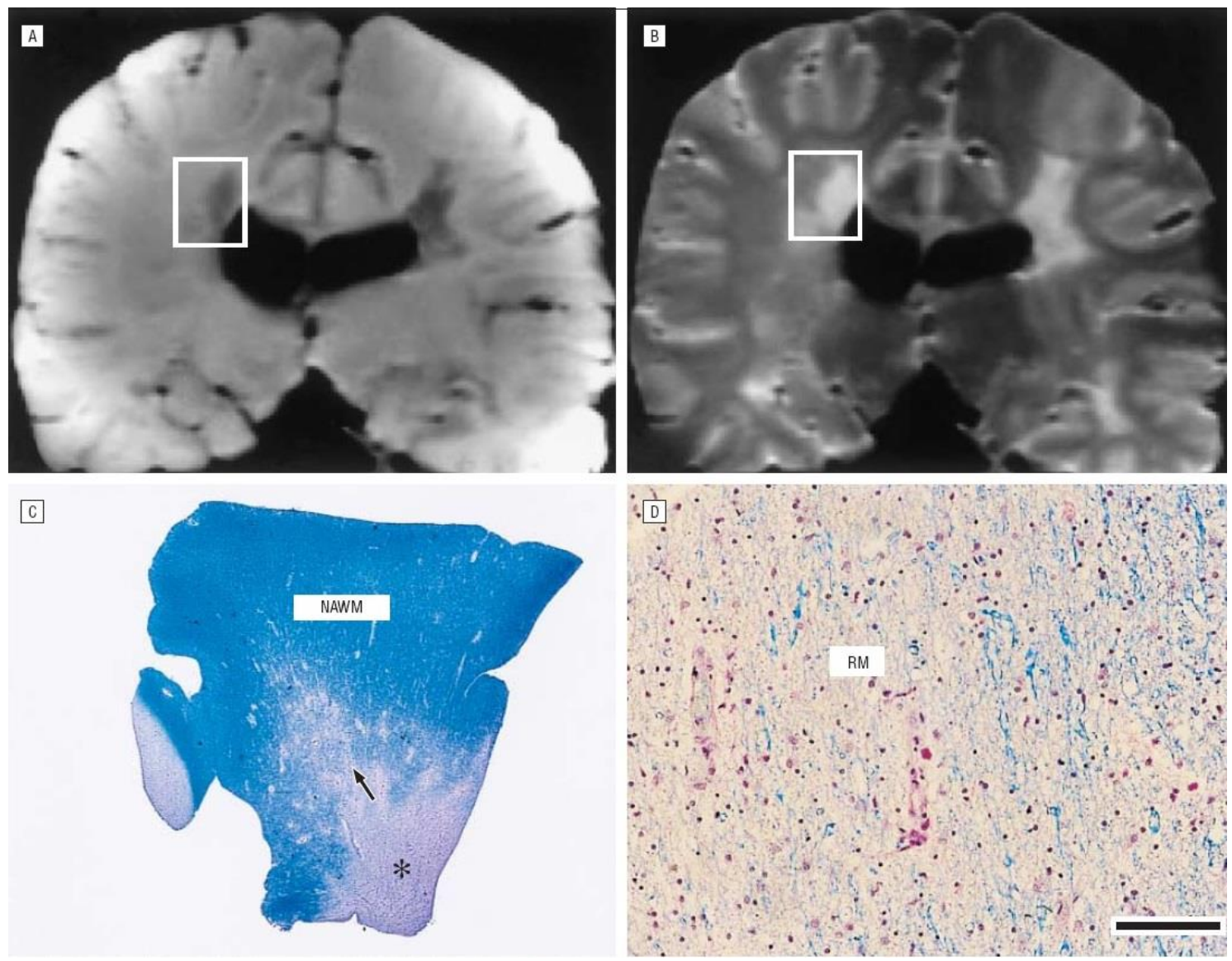

Figure 2.4: Remyelinated WM lesion. A and B, Coronal post-mortem T1-weighted (A) and T2-weighted (B) MRI. The frames contain a lesion that is mildly hypointense on T1. The lesion was dissected and analysed histologically. C, Photograph of a LFB stained, paraffin-embedded brain tissue section matching the frame on the MRIs contains a partially remyelinated lesion (arrow) confined to the border of an active demyelinated lesion in the white matter (asterisk). D, Photomicrograph of the edge of the demyelinated MS lesion showing reduced LFB staining in the remyelinated (RM) area (scale bar $=50 \mu \mathrm{m})$. (Barkhof F et al. Arch Neurol. 2003) 


\subsection{Grey matter pathology}

Although MS has been regarded as a disorder predominantly affecting the WM, interest in GM involvement has been increasing since the discovery of GM damage due to $\mathrm{MS}^{38,39}$. In the initial phases of MS, specific periventricular focal WM lesions appear; demyelination of the GM is relatively sparse in the initial phases of the disease but then rapidly increases as the disease progresses. Extensive demyelination in the cortex is associated with a more progressive phase of the disease.

GM damage can be directly viewed thanks to post mortem studies. The amount of cortical demyelination was reported to be different in relation to the disease course $^{29}$. In particular, demyelination in the cerebral cortex was mainly a feature in patients with SPMS and PPMS. Demyelination mainly affected the subpial layers of the cerebral cortex and was associated with mononuclear inflammatory infiltrates in the meninges.

In addition, MRI of the brain of patients with MS shows profound atrophy of the GM, in particular of the cerebral cortex, which is mainly present in patients with long standing progressive disease ${ }^{40}$. Another abnormality is a decrease in signal on T2-weighted images of deep grey matter structures, which probably reflects increased iron deposition ${ }^{41,42}$. In order to visualize cortical lesions, advanced multi-contrast three dimensional imaging confirmed the usefulness of double inversion recovery imaging, showing that it permits the highest detection of cortical lesions when it is compared with other sequences ${ }^{43}$. 


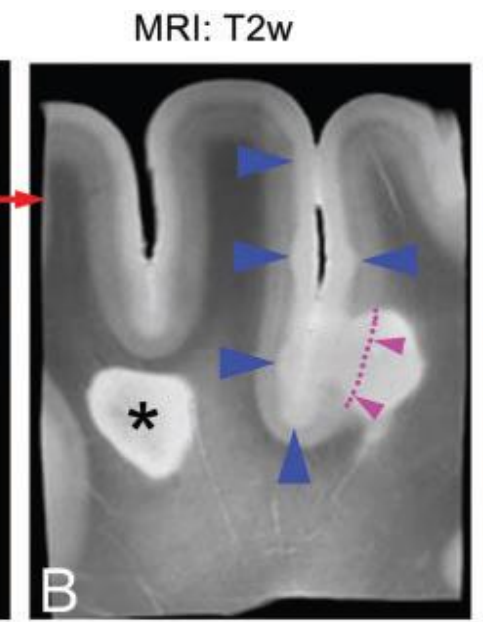

Figure 2.5: Correlation of MRI and histology in post-mortem brain of a patient with MS. Asterisks indicate a white matter MS lesion. Red and blue arrows reveals two cortical grey matter lesions. On $\mathrm{C}$, the tissue was processed for embedding in paraffin and stained for myelin basic protein. (Schmierer K, Parkes HG, et al. Brain 2010).

\subsection{Measuring Disability}

Until 1950, the evaluation of clinical change and the change with therapy was general and subjective and required a decision by the physician and the patient about whether things were better, the same, or worse. In 1950, there was an attempt to make an objective measure in a clinical trial in $\mathrm{MS}^{44}$ and in 1951, there was a first scale that could be applied to any clinical therapeutic trial ${ }^{45}$. This was an important concept that moved away from a general subjective decision to a more systemized approach. Although it was a rough scale, with a complex system of 30 neurological signs and disabilities, with variable numerical values, some repetitions, and a score that came from adding the 30 numbers, it opened the door to the development of better measures. The refinement of such measures continues to evolve with experience in continuing clinical trials. 
In 1955 and 1961, Kurtzke put forward a new scale for evaluating disability in multiple sclerosis, which he developed for a study of a therapy initiated in $1953^{46}$. The Disability Status Scale (DSS) became widely used, and in 1983, this evolved into the Expanded Disability Status Scale (EDSS), which is used in virtually all clinical trials. There were some problems with the original DSS scoring, with insensitivity in the mid-range of functional changes, so Kurtzke proposed the EDSS ${ }^{2}$.

\subsubsection{EDSS}

The EDSS is the most widely used scale and considers how the white and grey matter pathologies could affect eight functional systems: pyramidal, cerebellar, brainstem, sensory, bowel \& bladder, visual, cerebral, and other. It is a 20 -step scale of disease severity ranging from 0 (normal) to 10 (death due to MS). Between 0 and 3.5 , the score is based on the individual scores assigned to the eight functional system according to findings of the neurological examination. Between 4.0 and 5.5, the score is based on the distance the subject can ambulate; 6.0 represents the use of unilateral assistance such as a cane to walk; 6.5 represents the need for bilateral assistance such as a walker. Scores from 7.0 to 9.5 represent increasing degrees of immobility and dependence (Table 2.1).

The time spent by a patient at a given level of disability varies with the score. Thus, for patients with scores of 4 or 5 , median time spent at these levels was 1.2 years, whereas for those at 1 , median time to stay at that level was 4 years, and at 6, 3 years. These results have powerful implications for the conduct of clinical studies with respect to patient selection, stratification, and duration of follow-up: if many 
patients of 1 or 6 are included, little movement is seen in a group followed for 1 or 2 years.

Nevertheless, the EDSS is not an equivalent to the MS disease outcome. Some clinical practices never measure this score of the patient. But everyone agrees it is the clinical condition of the patient. It tends to predict future status over reasonable time domains, and has grown to become the outcome measure of choice in clinical trials. However, it has long been known that the EDSS is somewhat unreliable from visit to visit, varying in general by one point or more and yielding slightly more than $50 \%$ agreement from visit to visit. MS clinical researchers introduced the concept of sustained change in part to avoid this well-known unreliability of the EDSS. Anyway, the EDSS remains the unchallenged standard outcome for trials.

\subsubsection{T25FW}

Another score was developed in order to improve the outcome measure for MS clinical trials. The Multiple Sclerosis Functional Composite (MSFC) is a unified score representing the combination of results from three tests: the 9-Hole Peg Test, the Timed 25-Foot Walk (T25FW) and the 3-second Paced Auditory Serial Addition Test. These performance tests are combined to form a single score. The MSFC score incorporates three clinical dimensions representing arm, leg and cognitive function to create a single score that can be used to detect change over time in a group of MS patients. No measure of visual function was found at the time that could be used in the MSFC. Recent developments in other measures provide a potential important addition to the MSFC in the visual domain. 
In particular, the $\mathrm{T} 25 \mathrm{FW}$ measures the time a patient takes to walk 25 feet at maximum speed. As part of the MSFC, the T25FW is currently the most widely implemented method to objectively quantify gait disability in clinical MS trials and, to a lesser extent, clinical practice ${ }^{3,47}$. Although an excellent method of quantifying overall gait disability, the $\mathrm{T} 25 \mathrm{FW}$ measures only the time taken to walk a set distance $^{48}$.

Clinically meaningful functional benchmarks can be related to this score. Therefore, patients who take longer than 6 seconds to complete the test are more likely to use a cane and need assistance with daily activities than those who can walk this distance more quickly, and that a speed of 8 seconds or longer could mean facing additional social, occupational and ambulatory difficulties. These benchmarks, like the 6 seconds cut-off, can be used as research tools or for drug development ${ }^{49}$. 
Table 2.1: Neurologic disability classification: Expanded Disability Status Scale.

\begin{tabular}{|c|c|c|c|}
\hline Score & Disability description & $\begin{array}{l}\text { RRMS: } \\
\text { average time } \\
\text { until onset of } \\
\text { symptoms }(*)\end{array}$ & $\begin{array}{l}\text { CPMS: } \\
\text { average time } \\
\text { until onset of } \\
\text { symptoms }(*)\end{array}$ \\
\hline 1 & $\begin{array}{l}\text { No disability and minimal neurologic } \\
\text { symptoms. }\end{array}$ & $\begin{array}{l}11.4 \text { years } \\
\text { from Score } 1 \\
\text { to Score } 4\end{array}$ & $\begin{array}{l}0 \text { years from } \\
\text { Score } 1 \text { to } \\
\text { Score } 4\end{array}$ \\
\hline 2 & $\begin{array}{l}\text { Minimal disability in one or two } \\
\text { functional areas. Slight weakness or } \\
\text { stiffness, mild walking impairment or vi- } \\
\text { sual disturbances. }\end{array}$ & & \\
\hline 3 & $\begin{array}{l}\text { Moderate disability in one functional } \\
\text { area, such as vision or the urinary tract, } \\
\text { and possibly more than one minimal } \\
\text { disability in several others. Either a part } \\
\text { of one of the limbs or a whole side can be } \\
\text { partially paralyzed. May stagger at times. }\end{array}$ & & \\
\hline 4 & $\begin{array}{l}\text { Disability is relatively severe but there is } \\
\text { full ability to walk without aid. Patients } \\
\text { are self-sufficient and can be active } 12 \\
\text { hours a day and carry on normal } \\
\text { activities. Can walk without aid or rest } \\
\text { for } 300 \text { to } 500 \text { meters. }\end{array}$ & & \\
\hline 5 & $\begin{array}{l}\text { Disability is severe enough to impair or } \\
\text { even preclude a full day's activities. Able } \\
\text { to walk unaided and without rest for } 100 \\
\text { to } 200 \text { meters. }\end{array}$ & $\begin{array}{l}23.1 \text { years } \\
\text { from Score } 1 \\
\text { to Score } 6\end{array}$ & $\begin{array}{l}7.1 \text { years from } \\
\text { Score } 1 \text { to } \\
\text { Score } 6\end{array}$ \\
\hline 6 & $\begin{array}{l}\text { Can walk unaided for about } 100 \text { meters } \\
\text { only with assistance or devices, such as } \\
\text { two canes, crutches, or braces. }\end{array}$ & & \\
\hline 7 & $\begin{array}{l}\text { Mostly restricted to wheelchair, although } \\
\text { can manage the wheelchair and leave it } \\
\text { unassisted. Can walk with aids no further } \\
\text { than about five meters. }\end{array}$ & $\begin{array}{l}33.1 \text { years } \\
\text { from Score } 1 \\
\text { to Score } 7\end{array}$ & $\begin{array}{l}13.4 \text { years } \\
\text { from Score } 1 \\
\text { to Score } 7\end{array}$ \\
\hline 8 & $\begin{array}{l}\text { Mostly restricted to wheelchair or even } \\
\text { bed, but still has effective use of arms } \\
\text { remains and able to perform many self- } \\
\text { care functions. }\end{array}$ & & \\
\hline 9 & $\begin{array}{l}\text { Bedridden. Patient can communicate or } \\
\text { eat. }\end{array}$ & & \\
\hline 10 & Fatality occurs from complications. & & \\
\hline
\end{tabular}


Table 2.2: Multiple Sclerosis Functional Composite.

\begin{tabular}{|l|l|}
\hline Timed 25 Foot Walk & $\begin{array}{l}\text { The patient may use any walking aids needed and is } \\
\text { timed over a } 25 \text { feet course twice. The times are } \\
\text { averaged. }\end{array}$ \\
\hline 9 Hole Peg & $\begin{array}{l}\text { This is a timed test of upper extremity function and } \\
\text { consists of a block with nine holes and pegs. The time } \\
\text { it takes to insert all nine pegs, one at a time, and then } \\
\text { remove them using each hand separately. It is done } \\
\text { twice with each hand and the times averaged. }\end{array}$ \\
\hline Paced Auditory Serial Addition & $\begin{array}{l}\text { The patient listens to a tape in which a single digit } \\
\text { number is called out every } 3 \text { seconds. The task is to } \\
\text { add the last two digits spoken. This involves stating } \\
\text { the sum of the first two digits, dropping the sum, } \\
\text { adding the second and third digits and repeating this } \\
\text { with the next spoken digit. }\end{array}$ \\
\hline
\end{tabular}




\section{Chapter 3}

\section{Magnetic resonance imaging}

\subsection{Introduction}

MRI is a medical diagnostic technique that creates images of the internal structure and functionality of the human body. It uses the principle of nuclear magnetic resonance (NMR) which is a physical phenomenon used originally to investigate chemical and physical properties in atoms and molecules. The first magnetic resonance image was published in $1973^{50}$ when Paul C. Lauterbur spatially encoded the NMR signal in order to create images applying magnetic linear gradient fields. The first studies performed on humans were published in $1977^{51,52}$.

MRI provides greater contrast between the different soft tissues of the body than X-ray based techniques like Computed Tomography (CT) and this property makes it very useful in neurological, musculoskeletal, cardiovascular and oncological imaging. MRI is very good for distinguishing different parts of the brain and the spinal cord and for detection of tissue pathology. For example, MS diagnoses now are based usually on a typical pattern of lesions on MRI showing dissemination in space and with evidence for dissemination in time. MRI is the most sensitive method 
for revealing asymptomatic dissemination of lesions. Sensitivity to evolution of MRI lesions has made MRI abnormalities invaluable criteria for the monitoring of MS.

\subsection{Basic principles}

MRI uses non-ionizing radiation and is a non-invasive technique. The signals arise from the NMR properties of tissues. The scans are made by measuring the local response of the nuclei set into a constant magnetic field and excited with an electromagnetic field of appropriate frequency. There nuclei must be abundant enough to be detected above the background noise and can be performed only on atoms with a non-zero nuclear spin, i.e., with either an odd atomic number or with an odd mass number like in the case of ${ }^{1} \mathrm{H},{ }^{13} \mathrm{C},{ }^{19} \mathrm{~F}$, and ${ }^{31} \mathrm{P}$ nuclei.

Since a magnetic field is present whenever there are circulating charges, the nucleus must possess a microscopic magnetic field, which has a magnetic moment vector. If the sample volume is placed in a magnetic field, $B_{0}$, the microscopic spins tend to align with the applied external field and the spin system becomes magnetized. This can be modelled using a bulk magnetization vector, $\vec{M}$. $B_{0}$ applies a moment to the spins, thus modifying their magnetic moment. Spins will experience a precession around the $B_{0}$ axis. The angular velocity is given by:

$$
\Omega=\gamma B_{0}
$$


Where $\Omega$ is the Larmor frequency and $\gamma$ is the gyromagnetic ratio. Within the $B_{0}$ magnetic field, there are more spins aligned parallel to the field than spins aligned anti-parallel or against the field. Due to this excess of parallel spins, net magnetization $\vec{M}$ has a longitudinal component along the direction of the external magnetic field $B_{0}$.

As spins do not rotate in phase, the sum of all the microscopic transverse magnetizations of each spin is a null transverse macroscopic magnetization.

\subsubsection{RF excitation and relaxation}

When the spin system is excited by a resonant radio frequency (RF) pulse $B_{1}$ linearly polarized along the $\mathrm{x}$-axis (reference system $x_{0}, y_{0}, z_{0}$ ) rotating at the Larmor frequency around the z-axis, which is the same than the external magnetic field $B_{0}$, the total magnetization vector $\vec{M}$ will precess around $x_{0}$ with angular velocity:

$$
\omega_{1}=-\gamma B_{1}
$$

This motion of $\vec{M}$ is called forced precession, because it occurs in response to a deposition of RF energy that comes from outside the sample. The forced precession will lead to a transverse component $M_{x y}$ of $\vec{M}$. The actual evolution of $\vec{M}(\mathrm{t})$ is a spiral from the z-axis toward the xy-plane in a clockwise orientation when viewed from the positive z-axis. The inclination angle of $\vec{M}$ with its equilibrium direction is called flip 
angle. According to thermodynamics, once the pulse $B_{1}$ is removed, $\vec{M}$ will be back to the earlier equilibrium state which is called relaxation. During this relaxation, electromagnetic energy is transmitted in form of RF emission which is known as NMR signal.

Longitudinal relaxation is due to energy exchange between the spins and surrounding (spin-lattice relaxation), re-establishing thermal equilibrium. As spins go from a high energy state back to a low energy state, RF energy is released into the surrounding lattice. The recovery of longitudinal magnetization follows an exponential curve. The recovery rate is characterized by the tissue-specific time constant $\mathrm{T} 1$. These values are longer at higher field strengths.

$$
M_{z}=M_{0}\left(1-\mathrm{e}^{-\frac{t}{T_{1}}}\right)
$$

Transverse relaxation results from spins getting out of phase. As spins move together, their magnetic fields interact (spin-spin interaction), slightly modifying their precession rate. These interactions are temporary and random. Thus, spin-spin relaxation causes a cumulative loss in phase resulting in transverse magnetization decay. Transverse magnetization decay is described by an exponential curve, characterized by the time constant $\mathrm{T} 2$. It is tissue specific and is always shorter than T1. Transverse relaxation is faster than longitudinal relaxation. T2 values are unrelated to field strength.

$$
M_{x y}=M_{x y_{0}} \mathrm{e}^{-\frac{t}{T_{z}}}
$$


Decay in the Transverse magnetization is determined by two factors: i. Molecular interactions and, ii. The inhomogeneity of the external magnetic field, $B_{0}$.

\subsection{Imaging principles}

If the applied field is simply the external magnetic field $B_{0}$ then all the spins possess the same resonance frequency. Therefore, it is not possible to distinguish the signals generated from different spatial locations. By making the magnetic field inhomogeneous, we take advantage of the fact that the resonant frequency of these nuclei is proportional to the applied field strength and that this frequency changes instantaneously with changes in the field strength. Creating an inhomogeneous field with a gradient plays a vital role in MRI where spatial location is achieved by applying linear gradient magnetic fields in addition to $B_{0}$.

In order to encode the spatial location of each voxel, firstly a desired slice of voxels is selected and a selection gradient is applied perpendicular to the desired slice plane. This gradient is added to $B_{0}$, so the protons present a resonance frequency variation proportionate to the selection gradient. A RF wave is simultaneously applied, with the same frequency of the protons in the desired slice plane. This causes a shift in the magnetization of the protons on this plane only. Then, within the selected volume, the position of each voxel must be encoded by applying a phase encoding gradient and a frequency encoding gradient. The phase encoding gradient modifies the spin resonance inducing dephasing, which persists after the gradient is interrupted. Thus, protons will precess at the same frequency but with different phases. This phase difference lasts until the signal is recorded. Finally the frequency 
encoding gradient is applied when the signal is received, thus modifying the Larmor frequencies throughout the time of application.

\subsection{MRI sequences}

The NMR signal emitted by the relaxing protons is acquired using a RF coil. These coils serve to induce spin precession and to have currents induced in them by the spin system. Coils are sensitive to variations of transverse magnetization vector. The decay of $M_{x y}$ induces a sinusoidal FID, which depends on T1, T2, and $\rho$, which is the proton density of the tissue. Contrast in MRI is produced by manipulating the RF excitation pulse sequence to produce MR signals that are influenced by different weighted contributions of $\mathrm{T} 1, \mathrm{~T} 2$, and $\rho$. The architecture of a sequence consists of a combination of radiofrequency pulses and gradients. Different sequences are designed in order to highlight the signal of a particular tissue, while limiting both acquisition time and artifacts, without altering the SNR. The two main sequence families are the spin-echo and the gradient-echo. Each of them is characterized by the type of echo recorded. Besides, numerous variations have been developed within each of these families.

\subsubsection{Spin echo sequences}

Transverse relaxation, characterized by the time constant $\mathrm{T} 2$, is a random phenomenon. The experience shows that the FID is faster than that predicted by T2. 
This is due to fixed perturbations in the main magnetic field $B_{0}$. This relaxation time

is modelled as $T_{2}^{*}$ :

$$
\frac{1}{T_{2}^{*}}=\frac{1}{T_{z}}+\frac{1}{T_{2}^{r}}
$$

Where it can be seen the combination of two effects: the T2 decay itself and the dephasing due to magnetic field inhomogeneities $T_{2}^{I}$. These fixed perturbations cause the precession of some spins to speed up and others to slow down according to the nominal rate of rotation predicted by the Larmor frequency. In a brief period, spins are largely dephased. This process can be assessed by inducing rephasing using a shortduration $\pi$ pulse, forming a spin echo; a spin echo is the signal that is generated by the transverse spins recovering their coherence by the application of a $\pi$ pulse. The time interval from the initial $90^{\circ}$ pulse to the formation of the spin echo is known as the echo time (TE). This series is repeated at each time interval or repetition time (TR). The signal from a spin echo sequence is

$$
S \alpha \rho\left(1-\mathrm{e}^{-\frac{T R}{T_{1}}}\right) \mathrm{e}^{-\frac{T E}{T_{2}}}
$$

\subsubsection{Gradient echo sequences}

In a gradient echo sequence, there is no refocusing $\pi$ RF pulse and the data are sampled during a gradient echo, which is achieved by dephasing the spins with a negatively pulsed magnetic field gradient before they are rephased by a gradient with opposite polarity to generate the echo. Besides the absence of the rephasing $\pi$ pulse, 
gradient echo sequences are characterized also by enhancing efficiency of the signal acquisition using a flip angle less than $90^{\circ}$. Therefore, the amount of magnetization

tipped into the transverse plane decreases. The consequence of a low-flip angle excitation is a faster recovery of longitudinal magnetization that allows a shorter TR/TE and increases efficiency of scanning. The advantages of low-flip angle excitations and gradient echo techniques are faster acquisitions, adjustable contrasts between tissues and a stronger NMR signal in case of short TR. The relaxation due to fixed causes is not reversed and the loss of signal results from $T_{2}^{*}$ effects. Gradient echo is more sensitive to magnetic susceptibility artifacts than spin echo. The signal arising from a gradient echo is

$$
S \alpha \rho\left(1-\mathrm{e}^{-\frac{T R}{T_{1}}}\right) \mathrm{e}^{-\frac{T E}{T_{2}^{*}}}
$$

\subsubsection{Inversion recovery sequences}

Inversion-recovery can be used to null the signal of a single tissue, starts with a $\pi$ inversion pulse which flips longitudinal magnetization. Longitudinal magnetization will increase to return to its initial state, passing through a null value. This can be used to assess the longitudinal relaxation time if, before spins reach equilibrium, a $90^{\circ}$

pulse is applied which rotates the longitudinal magnetization into the XY plane.

For imaging contrast, the inversion recovery sequence has the advantage that, choosing the appropriate TI, it can provide very strong contrast between tissues having different $\mathrm{T} 1$ relaxation times or to suppress tissues like fluid or fat. On the other hand, the disadvantage is that the additional inversion RF pulse makes this sequence less time efficient. According to which kind of tissue is suppressed, there 
are different sequences like short TI inversion recovery (STIR), where TI is chosen to suppress fat, and fluid attenuated inversion recovery (FLAIR $)^{53}$, where TI is chosen to suppress CSF.

\subsection{FLAIR}

Grey matter and white matter are characterized by a much shorter T1 value than CSF. This fact can be exploited choosing a TI, so the contribution of CSF is equal or at least close to zero. The maximum number of slices that can be acquired in one TR is determined either by the time period TI or by (TR-TI). A longer TR will result in a more complete recovery of magnetization, in better SNR and increased contrast-tonoise ratio between the lesion on one hand and grey and white matter on the other hand. 

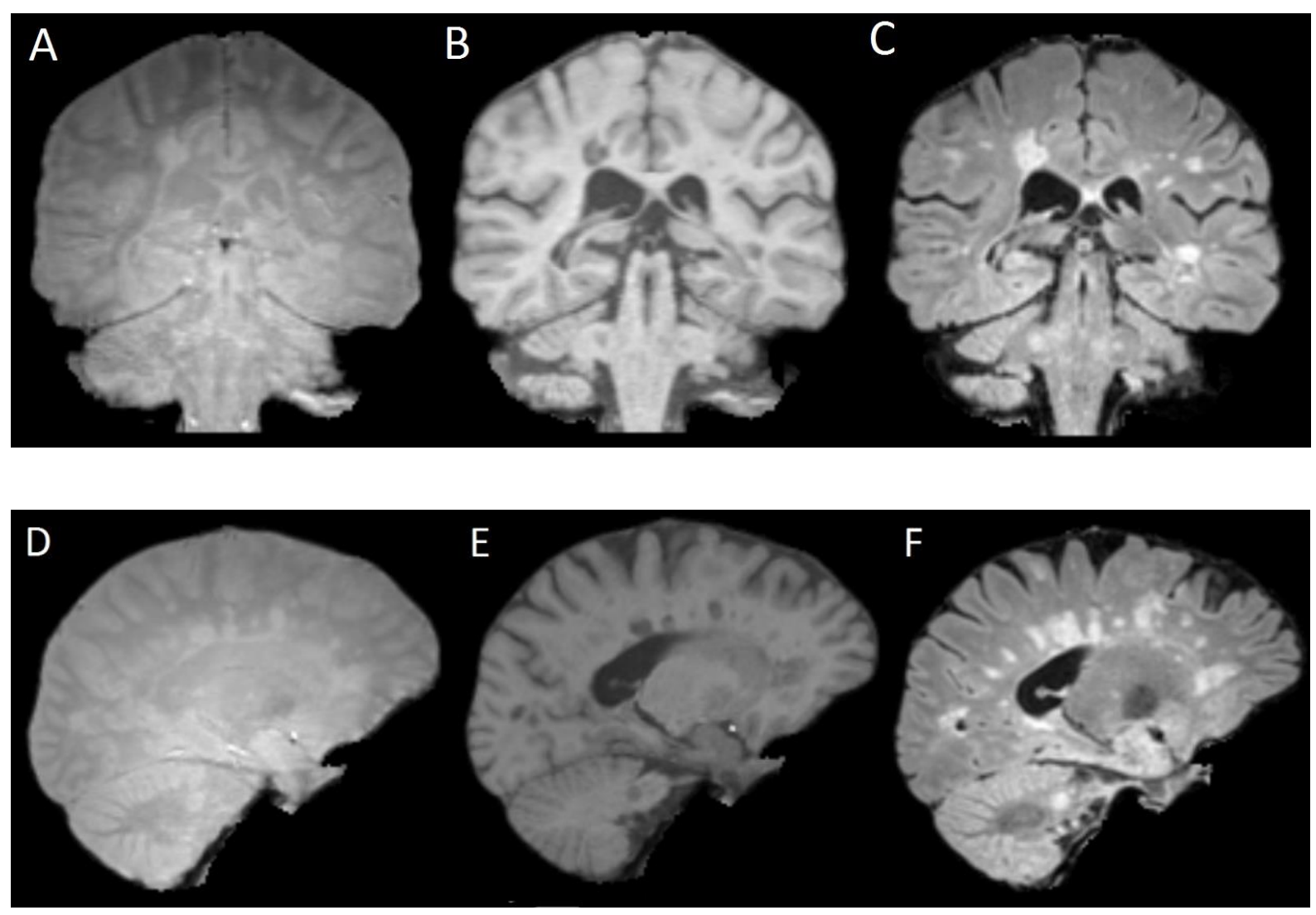

Figure 3.1: PDw (A, D), T1w (B, E) and FLAIR (C, F) images of a subject with multiple sclerosis. In FLAIR lesions have a brighter T2w signal. In the FLAIR image the surrounding CSF has been suppressed. PRC-REMS Imperial College London.

\subsection{Conventional MRI and disability}

The conventional MRI (cMRI) techniques used in MS in general are the sequences that are routinely used on a standard clinical MRI scanner. These typically include proton density, T2-weighted (T2W), SE, FLAIR among sequences for lesion identification, and gadolinium-enhanced T1-weighted (T1W) imaging for identifying new lesion activity.

cMRI is considerably more sensitive than CT imaging in delineating MS lesions ${ }^{54}$ but cMRI lacks pathological specificity ${ }^{55}$. Abnormalities identified on T2W 
images reflect altered tissue water content. This may be associated with axonal loss, gliosis, demyelination and edema, all of which can occur in MS lesions and all of which are associated with a variety of clinical impairment ${ }^{56}$. Due to the lack of specificity, in order to get a greater understanding of the natural history of the disorder, characterize the extent of tissue injury, and monitor the temporal evolution of both individual lesions and the overall disease activity, additional quantification techniques need to be employed. Plaques exhibit a wide range of values resulting in considerable different range of $\mathrm{T} 2$ relaxation times between acute and chronic lesions reflecting the substantial pathological heterogeneity ${ }^{55,57}$.

There is a strong correlation between $\mathrm{T} 2$ lesion load and clinical outcome in patients with clinically isolated syndromes ${ }^{56}$. However, in established MS, the correlation between total volume of $\mathrm{T} 2$ abnormalities and disability is modest or worse $^{58,59}$. This relates to the discrepancy between lesion site and locomotor function, which is most commonly assessed by EDSS. Clinical MRI correlations were demonstrated between relapse rate and number of lesions seen on $\mathrm{T} 2 \mathrm{~W}$ images, suggesting a role for MRI in monitoring disease progression, and between lesion number and volume and the presence or absence of treatment ${ }^{58}$.

Greater pathological specificity and sensitivity could be got with the development of advanced MRI techniques, like magnetization transfer imaging. 


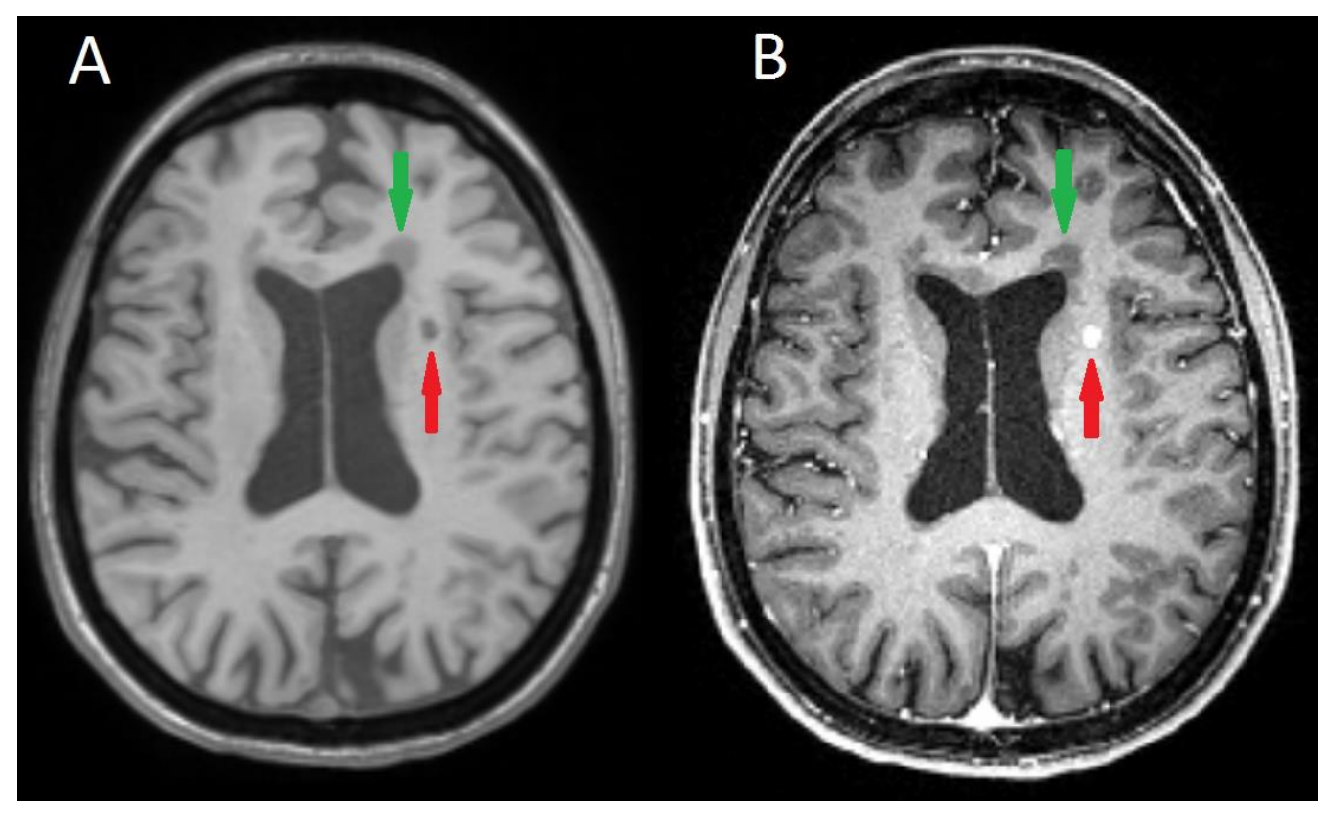

Figure 3.2: Axial T1 image (A) showing MS lesions. On post-gadolinium T1 image (B), a lesion shows an enhancement (red arrow). Intravenously administered, gadolinium $(\mathrm{Gd})$ chelate-based paramagnetic contrast agent markedly shorten the $\mathrm{T} 1$ of neighbouring water protons. The result is an increase in the signal from brain tissue where there is normally no blood-brain barrier (BBB) or where the barrier is compromised, allowing Gd to enter the brain. This gives rise to an increase in signal on the T1-weighted image. PRC-REMS Imperial College London. 


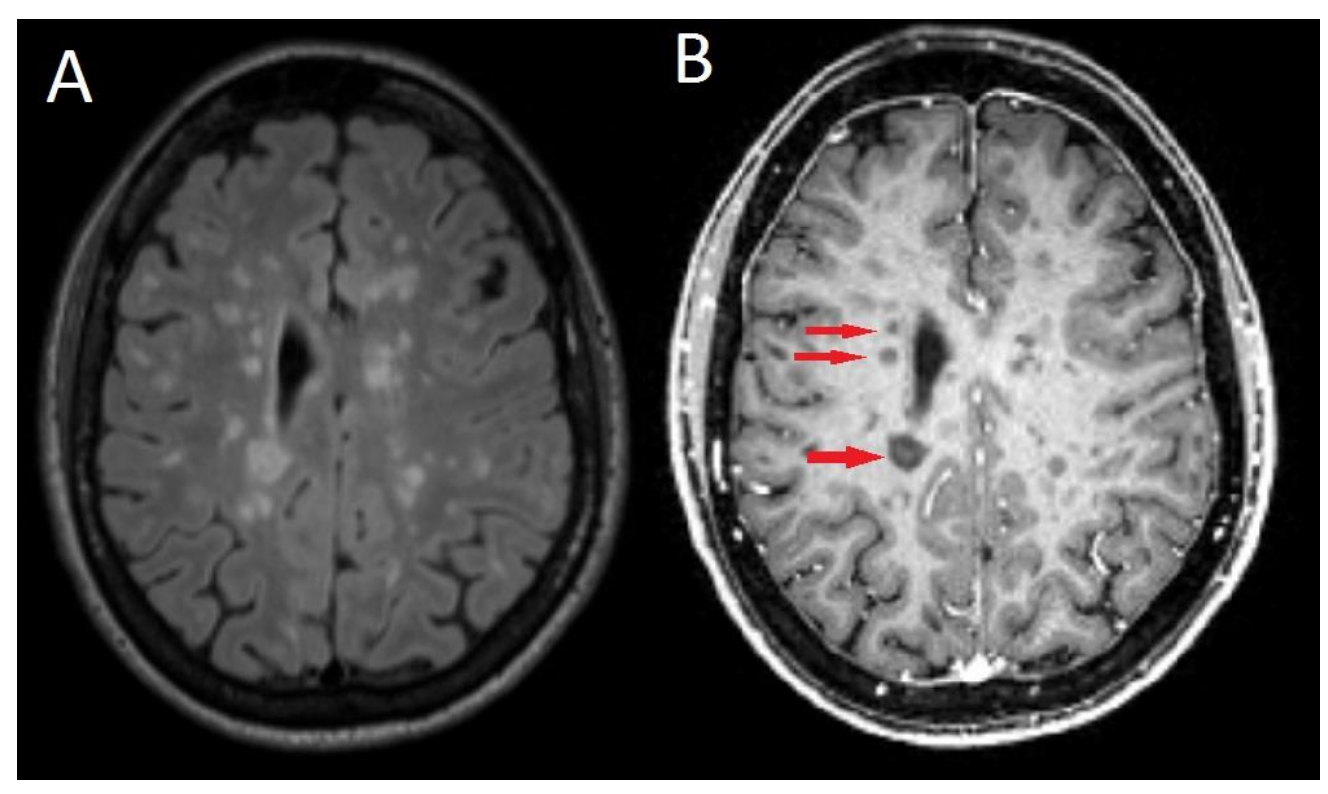

Figure 3.3: Axial T2 Flair (A) and T1 weighted (B) images with red arrows showing some hypointensities (black holes). T2W imaging is lack of pathological specificity, which provides only a dichotomous type of information: it discriminates between MS focal lesions and NAWM, but not between the type and degree of underlying pathologic substrate such as edema, inflammation, demyelination, remyelination, reactive gliosis and axonal loss. These different pathologies contribute differentially to the development of permanent disability. PRC-REMS Imperial College London. 


\section{Chapter 4}

\section{Magnetization transfer}

\subsection{Introduction}

Magnetization transfer contrast (MTC) is the result of selectively observing the interaction of bulk water protons with the protons contained in macromolecules of tissue. Magnetization transfer (MT) is based on well-defined biophysical and biochemical properties. Since different tissues have different macromolecular compositions, the transferring can differ widely, generating very high tissue contrast. MTC is obtained by combining saturation transfer techniques with standard MRI procedures.

The basic use of the saturation transfer technique in MR spectroscopy (MRS) was already described in 1963 by Forsén and $\operatorname{Hoffman}^{60}$, who used it to calculate the exchange rate of specific chemical reactions. In the 1970's, Edzes and Samulski ${ }^{36,61}$ proposed that cross-relaxation between ${ }^{1} H$ pools was a dominant relaxation mechanism in biological systems such as collagen and muscle. However, it was not until 1989 that Wolff and Balaban ${ }^{62}$ became the first to apply these principles to in vivo measurements. They calculated the pseudo-first-order rate constant for the 
transfer of magnetization in kidney and skeletal muscle, and showed images where the contrast was a function of magnetization transfer.

\subsection{Magnetization transfer physics: relaxation mechanisms}

In biological systems, hydrogen nuclei can be described as existing in two pools. The so-called free pool consists of relatively mobile protons in free bulk water and some lipid containing tissues. This pool has a narrow spectral line $(10-100 \mathrm{~Hz})$ and a relatively long T2 (>10-100 ms), and is labelled in mathematical descriptions as the A pool. With standard MRI this pool provides the bulk of the signal. The second pool or bound pool consists of restricted protons bound in proteins, other large macromolecules, and cellular membranes. This pool has a very broad spectral line $(10-50 \mathrm{kHz})$ and subsequently a very short $\mathrm{T} 2 \quad(<0.1 \mathrm{~ms})$, and is labelled in mathematical descriptions as the B pool. With cMRI this pool is not MR visible, because its signals are not directly detectable due to the very short T2. Both pools have approximately the same central resonance frequency (Figure 4.1) and that the only difference is the larger spread in resonance frequencies of the restricted protons.

Under normal conditions magnetization is exchanged from the free pool to the bound pool and vice versa, resulting in an equilibrium situation characteristic for that type of tissue. If the magnetization in either pool is perturbed (e.g. by the RF excitation pulses), changes occur in both pools. The normally observed spin-lattice relaxation of the free pool is a complex function of the recovery of both pools to the equilibrium state (relaxation coupling). A special situation occurs when the bound pool is selectively saturated. This will result in a difference in magnetization between the pools. Due to cross relaxation processes, magnetization is transferred from the free pool to the bound pool. 


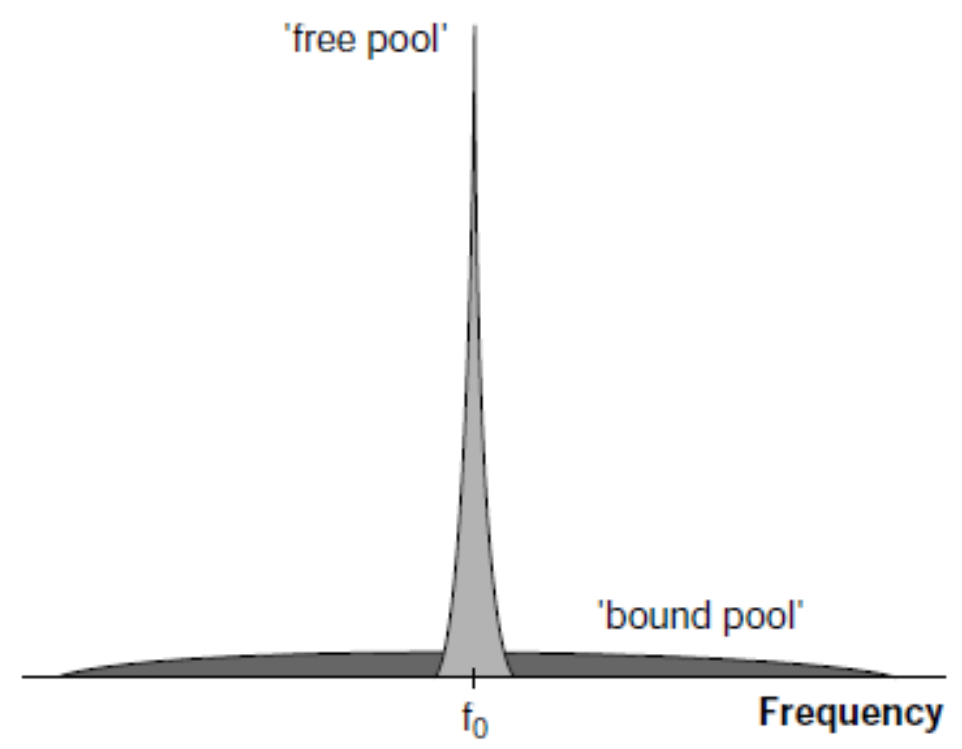

Figure 4.1: The spectral lines of the free pool and the bound pool are both centred at the same Larmor frequency. The free pool has a narrow line and the bound pool has a homogeneous broadened line.

\subsubsection{Cross relaxation: dipole-dipole interactions}

Although the magnetization transfer mechanism is relatively well understood at the tissue level, much less detailed knowledge is available on a cellular and molecular level. Advances in modelling of magnetization transfer effects will improve the detailed picture of the mechanism underlying this phenomenon ${ }^{63,64}$. The most comprehensive models, as summarized by Balaban and Ceckler ${ }^{65}$, describe the free proton pool and the macromolecule proton pool. The free pool is thought to include both mobile bulk water molecules and water molecules bound to the macromolecular surface, the so-called hydration water or surface water layer. These two water fractions exist in a state of rapid chemical equilibrium, such that all the tissue water can be thought of as a single proton pool. The surface layer of water communicates 
with the bulk water by diffusion ${ }^{66}$. The actual transfer of magnetization from the surface layer of water with the macromolecule protons occurs by means of dipoledipole interactions through space (cross relaxation).

Lipid bilayers are effective macromolecular structures for magnetization transfer between bound and free protons ${ }^{66}$. It has also been suggested that chemical exchange between protons in hydration water and macromolecule protons could be the primary mechanism for magnetization transfer. Furthermore, in model lipid

systems and other macromolecules, similar magnetization transfer effects have been observed independently of $\mathrm{pH}$ values from 5 to 8 . From these studies it is concluded that chemical exchange is not a significant factor in the magnetization transfer process $^{66}$.

\subsubsection{Saturating pulses}

Frequency selective RF pulses are transmitted at a frequency offset $\Delta \mathrm{f}$ with respect to the central ${ }^{1} H$ Larmor frequency (Fig 4.2). Using these off-resonance pulses it is possible to substantially saturate the broad resonance of the bound pool while hardly affecting the free pool. Such a pulse will not only irradiate the spins with the specific off-resonance frequency. Due to spin diffusion all the macromolecule protons will be affected. As a consequence, signal of the water peak will be reduced as a result of the magnetization transfer process. 


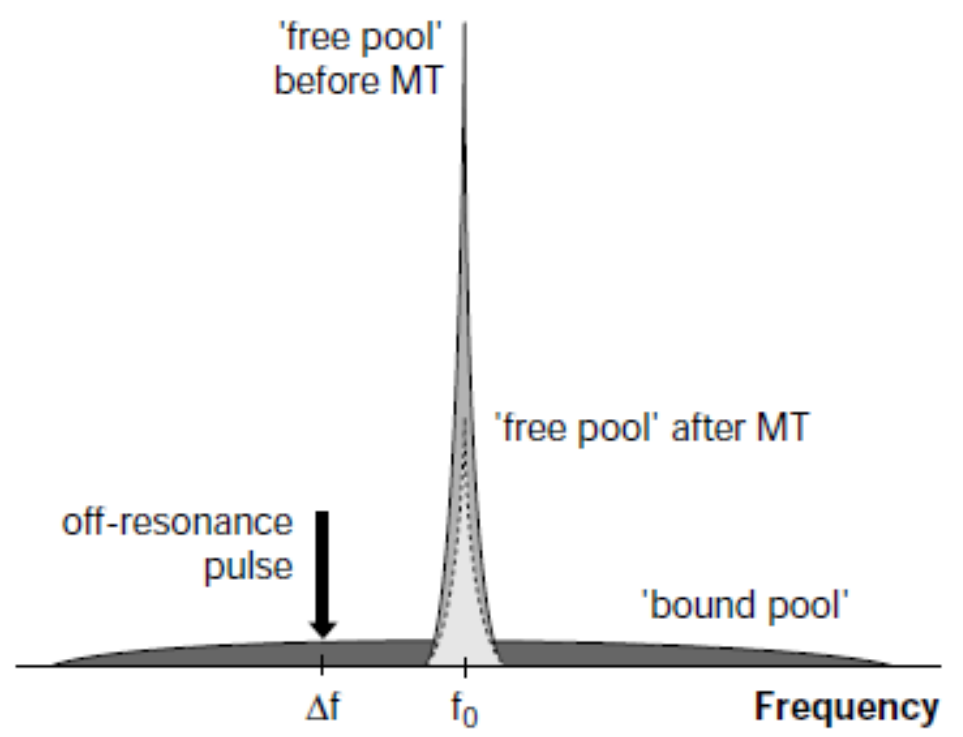

Figure 4.2: Using off resonance pulses it is possible to saturate the broad resonance of the bound pool while hardly affecting the free pool. Due to spin diffusion all the macromolecule protons will be saturated. The lower water peak is the result of the MT process.

The off-resonance pulse is a single, shaped RF pulse (e.g. sinc-Gauss) with a relatively narrow excitation bandwidth (dependent on pulse shape and duration). Two critical parameters will affect the amount of saturation: the magnitude and the frequency offset of the pulse ${ }^{67,68}$. More B1 power will result in a better saturation, but is limited in practice by safety considerations, specific absorption rate (SAR). Decreasing the frequency offset will also create a better saturation of the bound pool, but values closer to the ${ }^{1} H$ Larmor frequency will also result in greater direct excitation of the free pool. 


\subsection{Magnetization transfer ratio: MTR}

MTR maps, which are derived from two MR images, are acquired without and with an off-resonance saturation pulse. The MTR is defined with the following equation ${ }^{69}$ :

$$
M T R=\frac{\left(M_{0}-M_{S}\right)}{M_{0}} 100
$$

In which $M_{o}$ is the intensity of a given voxel without the saturation pulse, and $M_{s}$ is

the intensity of the same voxel when the saturation pulse is applied. Thus, MTR represents the fraction of signal loss due to the complete or partial saturation of the bound proton pool, and ranges from near zero in the CSF to about $50 \%$ in tissue that contains a high proportion of bound water molecules.

\subsection{Magnetization transfer saturation: MTsat}

Once a brain examination could be completed in a time shorter than a patient's tolerance for the procedure, MR imaging acceptance began to grow. While scan times decreased with the technological improvements, the next major gain in speed came with the introduction of the fast low angle shot (FLASH) gradient echo method in $1986^{70}$. By reducing practical scan times, the FLASH method considerably increased MR applications.

Reducing TR is a simple way to reduce scan times, but its implications are complex. When TR is reduced, the magnetization from tissues with long T1 does not recover fully, and the MR signal is diminished. Because the signal strength depends on T1 as TR is shortened, the images become increasingly T1-weighted. Due to the strength of the MR signal depends on the projection of the magnetization on the transverse plane, large flip angles yield larger MR signals. An excitatory RF pulse which disturbs the magnetization to a lesser degree can be applied. Therefore, when 
small flip angles are used for excitation, there is relatively little loss of longitudinal magnetization and as a result, the tissues will re-magnetize more rapidly. The first imaging technique to utilize this reduced flip angle advantage was the FLASH method. Although small flip angles cannot yield signals as strong as those of large flip angles, it is possible at any TR to specify a flip angle, less than 90 degrees which will give the maximum signal possible at that TR. With FLASH it is possible to control the T1 contrast weighting with both flip angle and TR.

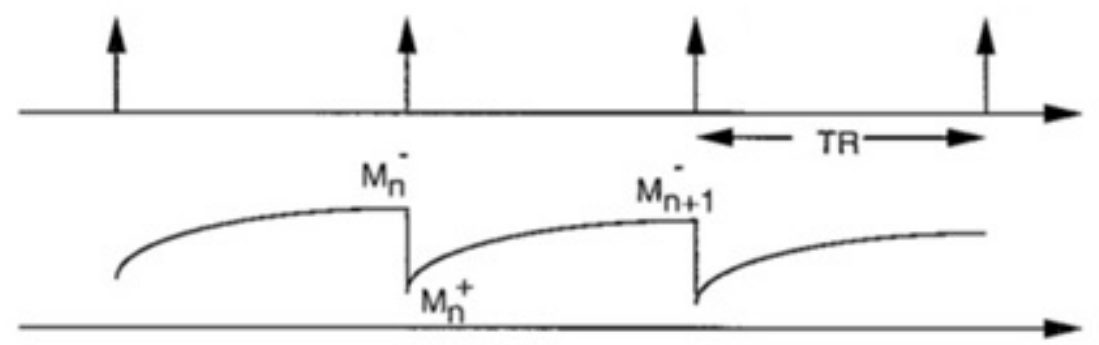

Figure 4.3: General excitation recovery sequence in which an $\alpha$ degree pulse is applied at a repetition time of TR. In this sequence $M_{z}$ does not reset to zero after each pulse.

The longitudinal components before and after the $\alpha$ pulse are (Figure 4.3):

$$
M_{n}^{+}=M_{n}^{-} \cos \alpha
$$

The longitudinal component will relax during the inter pulse interval:

$$
\begin{gathered}
M_{n+1}^{-}=M_{0}-\left(M_{0}-M_{n}^{+}\right) \exp \left(R_{1} T R\right) \\
M_{n+1}^{-}=M_{0}-\left(M_{0}-M_{n}^{-} \cos \alpha\right) \exp \left(R_{1} T R\right)
\end{gathered}
$$


The steady state is established when $M_{n+1}^{-}=M_{n}^{-}$. In this moment the longitudinal components prior to the $n^{\text {th }}$ and $(n+1)^{\text {th }}$ pulses are equal. If $M_{n}^{-}=M^{-}$:

$$
M^{-}=\frac{M_{0}\left(1-\exp \left(R_{1} T R\right)\right)}{1-\cos \alpha \exp \left(R_{1} T R\right)}
$$

The transverse component is the Ernst equation which describes the effects of excitation and longitudinal relaxation in the FLASH sequence:

$$
S(\alpha \cdot T R)=A \sin \alpha \frac{1-\exp \left(-R_{1} T R\right)}{1-\cos \alpha \exp \left(-R_{1} T R\right)}
$$

When flip angles ( $\alpha \ll 1 \mathrm{rad}$ ) are small and TR much shorter than longitudinal relaxation $\left(R_{1} T R \ll 1\right)$, the signal is an approximation of the Ernst equation:

$$
S(\alpha . T R) \approx A \alpha \frac{R_{1} T R}{\alpha^{2} / 2+R_{1} T R}
$$

When instead of just one excitation, there are two during $\mathrm{TR}=\mathrm{T} R_{1}+\mathrm{T} R_{2}$ :

$$
S_{1}=A \sin \alpha_{1} \frac{1-\exp \left(-R_{1} T R\right)-\left(1-\cos \alpha_{2}\right)\left[1-\exp \left(-R_{1} T R_{1}\right)\right] \exp \left(-R_{1} T R_{2}\right)}{1-\cos \alpha_{1} \cos \alpha_{2} \exp \left(-R_{1} T R\right)}
$$

Under the same approximation conditions for the flip angles and TRs, then the rational approximation of the Ernst equation 4.8 takes the form for each excitation:

$$
\begin{gathered}
S_{1} \approx A \alpha_{1} \frac{R_{1} T R}{\alpha_{1}^{2} / 2+\alpha_{2}^{2} / 2+R_{1} T R} \\
S_{2} \approx A \alpha_{2} \frac{R_{1} T R}{\alpha_{2}^{2} / 2+\alpha_{1}^{2} / 2+R_{1} T R}
\end{gathered}
$$

When by analogy to this dual excitation, there is an excitation and the second one is replaced by the MT pulse, then we have the MT FLASH sequence. The additional saturation due to MT is observed as a reduction in the steady state excitation signal: 


$$
S_{M T} \approx A \alpha \frac{R_{1} T R}{\alpha^{2} / 2+\delta+R_{1} T R}
$$

Where $\delta\left(M T_{\text {sat }}\right)$ is a term introduced to describe the reduction of the signal by the application of the MT pulse $(\delta>0)$. The parameters $\mathrm{A}$ and $R_{1}$ are calculated from two FLASH signals (PDw and T1w) at different excitation flip angles $\left(\alpha_{P D}, \alpha_{T_{1}}\right)$, repetition times $\left(T R_{P D}, T R_{T_{1}}\right)$ and without MT pulse :

$$
\begin{gathered}
R_{1}=\frac{1}{2 T} \frac{s_{T_{1} \alpha_{T_{1}}-S_{P D} \alpha_{P D}}}{S_{P D / \alpha_{P D}-}-S_{T_{1} / \alpha_{T_{1}}}} \\
A=\frac{S_{P D} \alpha_{P D} / T R_{P D}}{S_{T_{1} \alpha_{T_{1}}-S_{P D} \alpha_{P D}}}\left(\alpha_{T_{1} / \alpha_{P D}}-{ }^{\alpha P D} / \alpha_{T_{1}}\right)
\end{gathered}
$$

Finally, the $M T_{\text {sat }}$ can be calculated from equation 4.11:

$$
M T_{\text {sat }}=\left(A \alpha / S_{M T}-1\right) R_{1} T R-\alpha^{2} / 2
$$

Axial images of the MT FLASH and references PDw/T1w FLASH are shown in Figure 4.4. The MT saturation map exhibit a high T1-like contrast between WM, GM and CSF. By increasing the flip angle, but below the limit of rational approximation for the dual excitation of $25^{\circ}$, the MT related T2-like contrast was eliminated (Figure 4.5). 


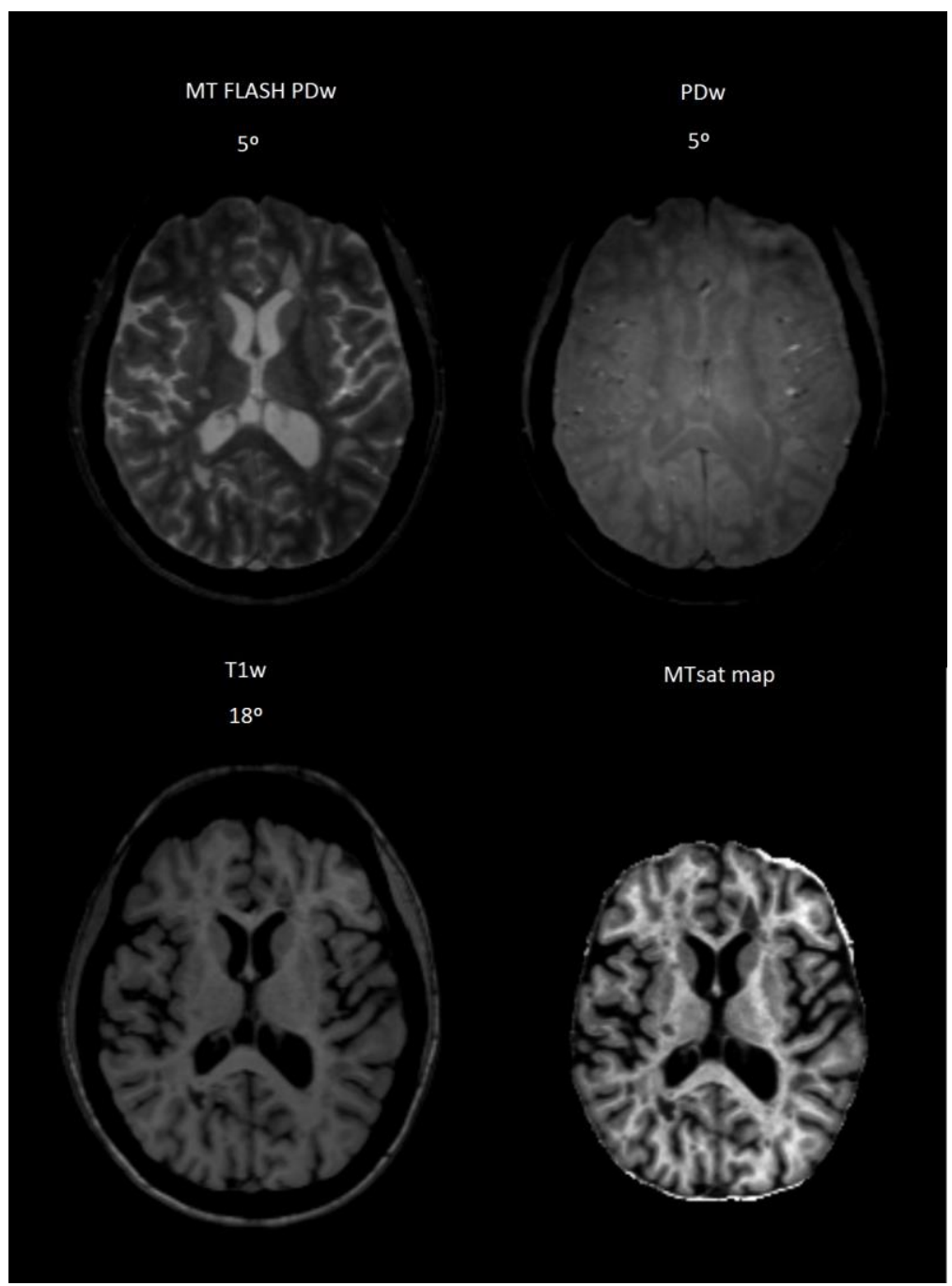

Figure 4.4: MT FLASH, PDw/T1w reference images, and MTsat map. The MT saturation map was obtained with the reference and the MT FLASH images using the equation 4.14. The T2-like weighting related to MT FLASH is appreciated when is compared to the PDw reference. PRC-REMS Imperial College London. 


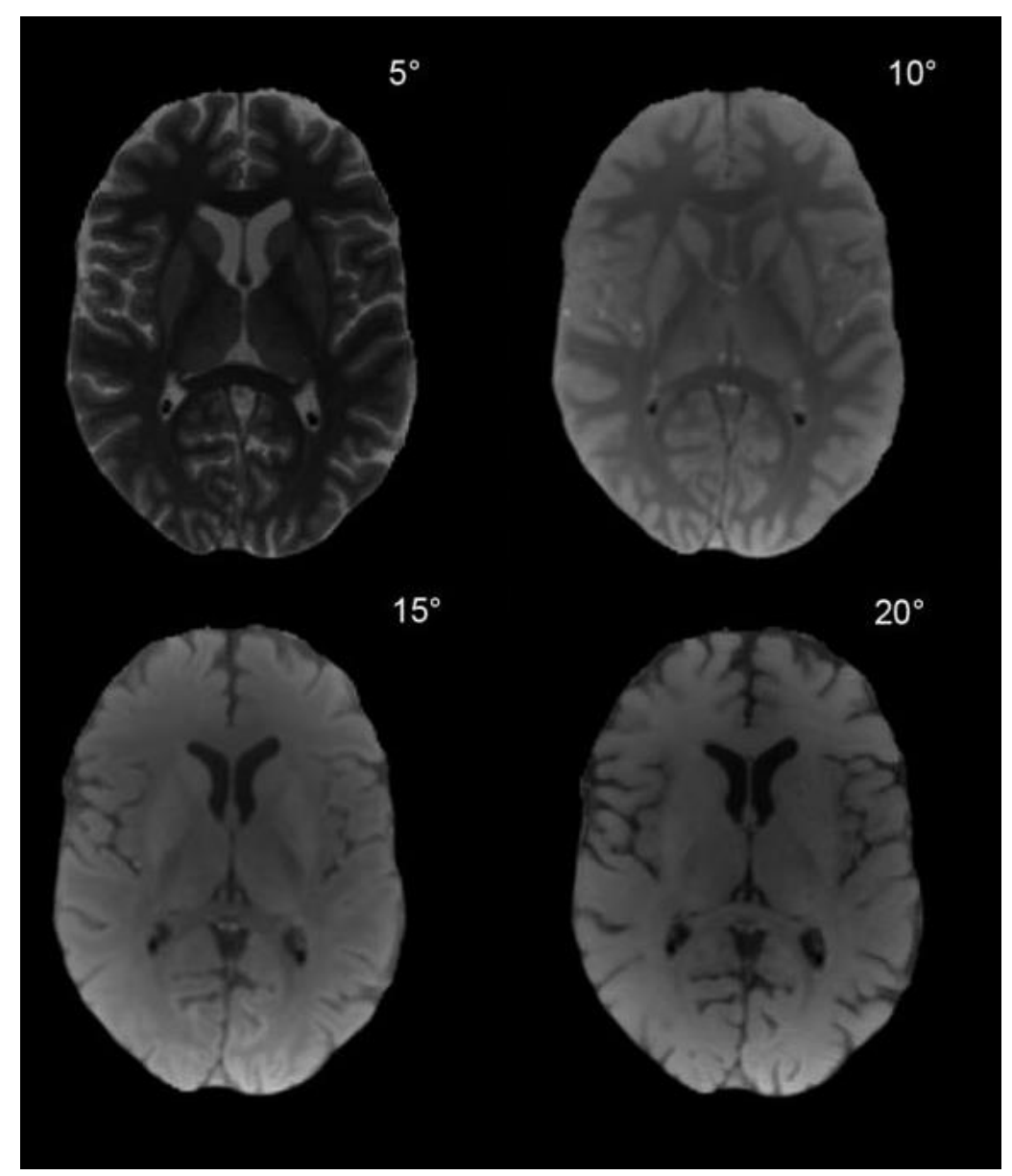

Figure 4.5: Axial MT FLASH images for four different flip angles. It is shown how the T2-like contrast is eliminated as the flip angle increases. (Helms $\mathrm{G}$ et al. ${ }^{71}$ )

The Figure 4.6 shows number of voxels against intensity of the signal (histogram) of the MTsat maps calculated from MT FLASH for different flip angles. Three separate modes can be seen. A separate CSF mode is only seen for $5^{\circ}$ and $10^{\circ}$ because the signal to noise ratio decreases with the signal at higher flip angles.

The relation between the flip angle and the magnetic field in the case of a Gaussian pulse is given by:

$$
\theta_{0}[\operatorname{deg}]=\frac{360 y B_{1 \max } \pi}{\sqrt{2 \pi}}
$$


For instance, an off resonance Gaussian MT pulse with a duration of $7.68 \mathrm{~ms}$ has a nominal flip angle of $500^{\circ}$ for $B_{1 \max }$ of $8.69 \mu \mathrm{T}$. The MTsat increases strongly with the energy of the MT pulse (Figure 4.7). The $180^{\circ}$ MT pulse cannot create separated modes in the histogram, and at $720^{\circ}$, the specific absorption rate (SAR) can exceed the safety limits. At decreasing frequency offsets (Figure 4.8), the peak values of MTsat in GM and WM increased due to a higher absorption. In order to reduce the direct absorption in the free water pool, large frequency offsets should be applied even if this means a smaller MT saturation.

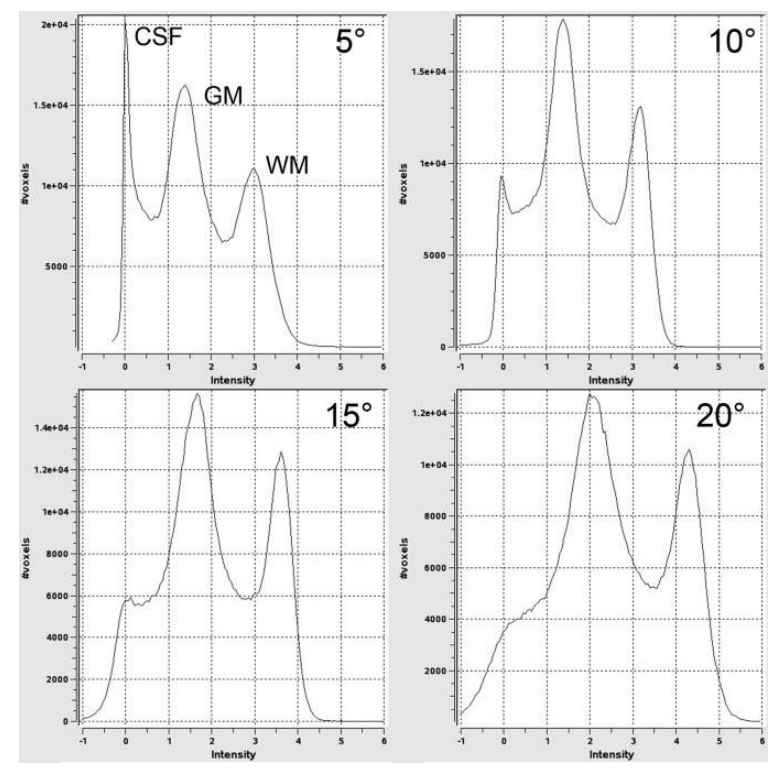

Figure 4.6: MTsat histograms from MT FLASH with $5^{\circ}, 10^{\circ}, 15^{\circ}$ and $20^{\circ}$ flip angle. The modes WM and GM are separated. The CSF mode appeared increasingly blurred at higher flip angle. (Helms $\mathrm{G}$ et al. ${ }^{71}$ ) 


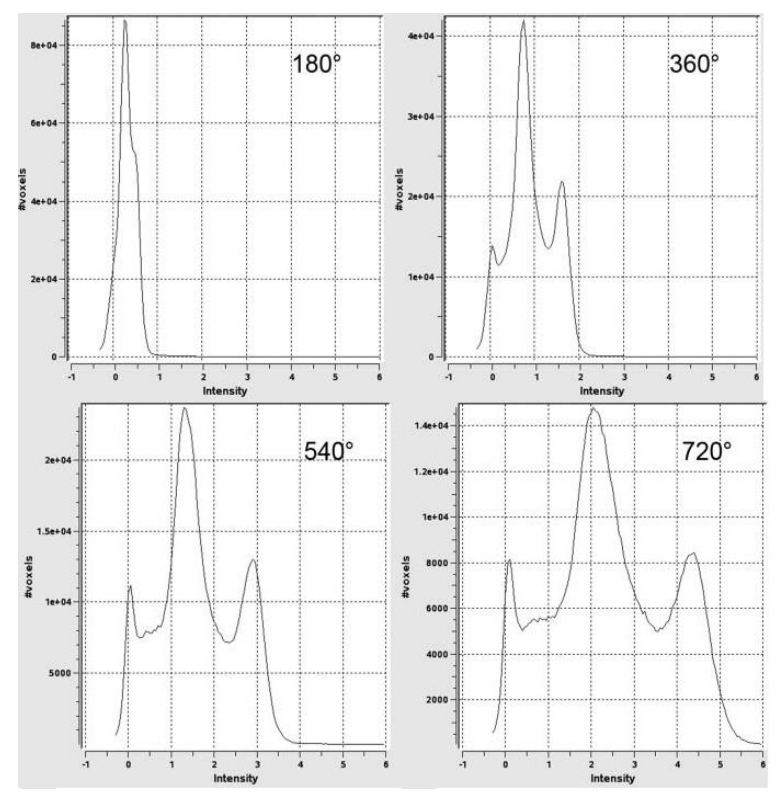

Figure 4.7: MTsat and MT pulse energy. MTsat histograms obtained with nominal $180^{\circ}, 360^{\circ}, 540^{\circ}$ and $720^{\circ}$ MT pulses. (Helms G et al. ${ }^{71}$ )

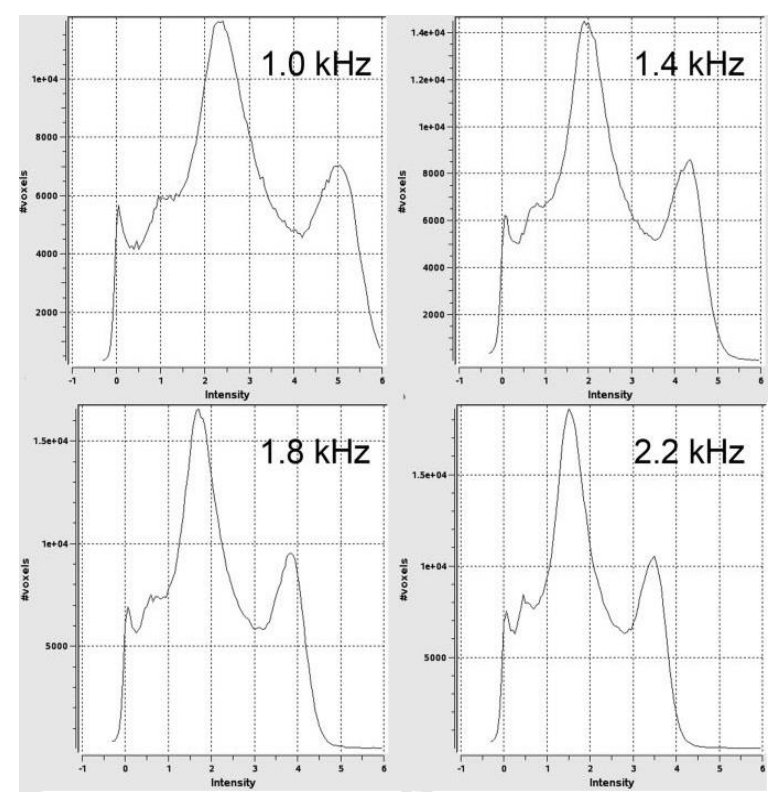

Figure 4.8: MTsat and frequency offset. These are MTsat histograms obtained with MT pulses of frequency offsets of 1.0, 1.4, 1.8 and $2.2 \mathrm{kHz}$. The separation of the major modes was about the same for $1.4,1.8$ and $2.2 \mathrm{kHz}$. (Helms G et al. ${ }^{71}$ ). 


\subsection{Magnetization transfer imaging and disability}

Although T2W scans play a major role in the assessment of MS lesion burden ${ }^{72-74}$, cross-sectional studies ${ }^{75}$ found that the magnitude of the correlation between clinical disability and brain T2W lesion load is only modest. The poor correlation is likely due to the extremely variable extent of the tissue damage of WM lesions visible on cMRI scans. Using MTR, it is possible to grade the extent of tissue damage of individual MS lesions and to detect additional pathology in otherwise NAWM. Monitoring individual lesion evolution specifically may be relevant for the understanding of MS pathophysiology, and as a new approach for assessing treatment efficacy, particularly for remyelinating therapies. In preliminary trials, this approach might indeed give information in relatively short periods of time about the efficacy of experimental treatment in preventing severe tissue destruction.

Lower MTR has been reported in hypointense lesions than in lesions that are isointense to NAWM on T1-weighted scans ${ }^{76-79}$, and MTR has been found to be inversely correlated with the degree of hypointensity ${ }^{76,78,80}$. On the basis of these results and of post-mortem studies ${ }^{55,78}$, it may be argued that fixed MS lesions with lower MTR are expressions of more severe demyelination and axonal loss. The MTR is lower for patients with more disabling MS courses ${ }^{35}$. Decreased MTR has also been found in NAWM areas that are adjacent to focal T2W lesions ${ }^{35,81,82}$ and in WM areas characterized by subtle and diffuse signal intensity changes on T2W images, referred to as dirty-appearing white matter (DAWM). These findings suggest that the actual size of MS lesions is greater than that visible on T2W images, and that evidence for demyelination detected by MT imaging might be relevant in determining a patient's disability. 
Post-mortem studies showed that neuropathological abnormalities can be detected in NAWM from patients with MS. These abnormalities include diffuse astrocytic hyperplasia, oedema and perivascular cellular infiltration. There may be abnormally thin myelin in the NAWM, and post-mortem studies also detected signs of axonal damage in NAWM. Such pathological abnormalities modify the relative proportions of mobile and macromolecule bound protons of the diseased tissue. Therefore, it is not surprising that MT imaging could be able to show microscopic damage in the NAWM which is not detected by $\mathrm{cMRI}^{35,81,82}$. Several of the pathological substrates above may contribute to the changes in NAWM. These include edema, marked astrocytes proliferation, perivascular inflammation and demyelination. All of these processes may account for an increased amount of unbound water and, as a consequence, determine MTR changes.

NAWM MTR values are altered in all of the major phenotypes of MS, these alterations include multiple cerebral regions ${ }^{35,81-83}$. Reduced MTR values have been found in several NAWM regions, even in patients with clinically definite MS and very few or no $\mathrm{T} 2$ visible lesions ${ }^{83,84}$. These observations have indicated the need to obtain more accurate estimates of the overall extent of NAWM damage in MS and, as a consequence, have led to the application of histogram analysis to all brain voxels on $\mathrm{cMRI}^{85}$.

The application of MT imaging to the assessment of MS damage in the spinal cord is needed in order to increase our understanding of the mechanisms leading to the development of irreversible disability in MS. Studies based on histogram analysis found that cervical cord of MS patients had lower MTR values than that of controls ${ }^{86}$. On the other hand, at the earliest clinical stage of MS, no difference in cervical cord 
MTR between patients and healthy controls was found, independently of the burdens of visible lesions seen on brain and cord scans ${ }^{87}$.

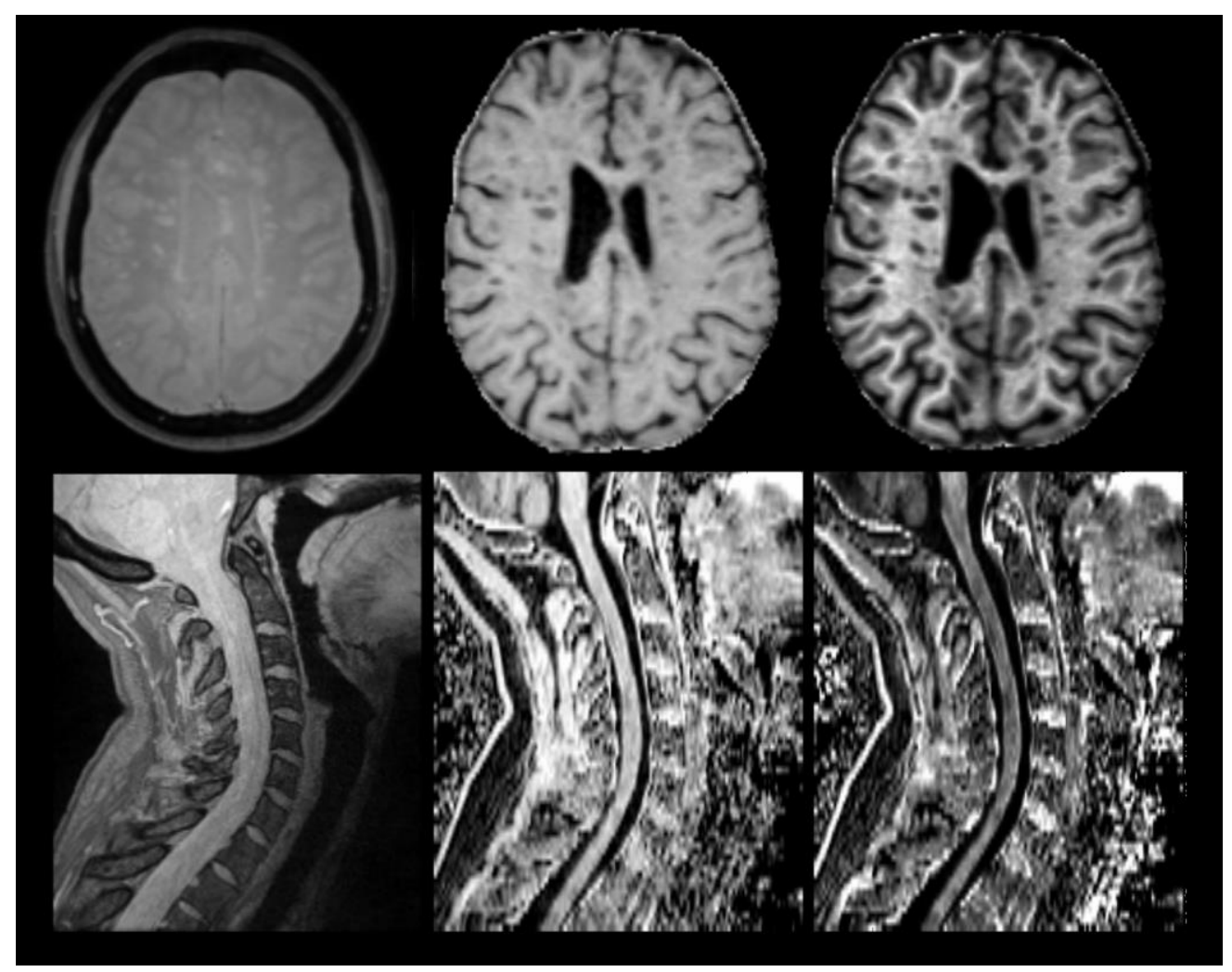

Figure 4.9: On the left, a conventional MRI proton-density weighted image in the brain and cervical spinal cord of a multiple sclerosis patient shows hyperintensities. The middle image shows an MTR map. The right image shows the MTsat map where the contrast in MT between grey and white matter is improved. PRC-REMS Imperial College London. 


\section{Chapter 5}

\section{Comparison of MTR and MTsat}

\subsection{Introduction}

Magnetization transfer (MT) is the nuclear magnetic resonance phenomenon in which spins in two or more distinct environments exchange their magnetization. MT imaging is based on interactions between protons in free fluid and bound protons from macromolecules. Magnetization transfer ratio (MTR) and magnetization transfer saturation (MTsat) are two MT imaging techniques which exploit the MT effect and are more sensitive to the neuropathology of multiple sclerosis (MS) than conventional MR techniques (cMRI). MTR is the percentage of reduction in the signal when the saturation is applied and among its dependencies is T1 relaxation time. It is not an absolute measure but is commonly taken as an index for the strength of the MT effect and as a parameter for assessing relative macromolecular content or myelination. The T1 dependency is one of the MTR limitations. In some circumstances, like in the case of demyelination, an increase in $\mathrm{T} 1$ can partially mask the decrease in macromolecular density, resulting in an apparent stability of the MTR. In MS lesions the decrease in the fraction of bound protons and the increase of T1 (relaxation of free 
water in absence of exchange) may partly counteract each other. Some lesion studies found that the bound fraction protons correlated strongly and negatively with $\mathrm{T} 1$, with the product being almost constant ${ }^{88}$.

MTsat is a novel semi quantitative parameter ${ }^{71,89}$ which comes from the attempt to separate T1 relaxation from the MT effect. MTsat reflects any additional saturation due to the MT pulse and appears to be sensitive to the presence of demyelination. Therefore, MTsat may be a sensitive parameter for discrimination of MS lesions and for grading pathological alterations in the white matter (WM) and the grey matter (GM).

An image voxel-by-voxel histogram provides an approximation of the continuous probability density distribution for discrete images. Therefore, an implicit hypothesis of MTR and MTsat histograms analysis is that both kinds of maps can be completely characterized by their probability density distribution. The first MTR histograms were published by McGowan, Grossman, van Buchem and colleagues in $1996^{90}$ and the first MTsat histograms were published by Helms, Dathe, Dechent and colleagues in $2008^{71}$. These tools for analysis offers the advantage of being a much less complicated and less time consuming method as compared with other techniques based on relaxation time measurements. Histograms allow the quantification of diffuse pathological changes in MS and provide insight into disease mechanism.

MS is an inflammatory demyelinating disorder of the central nervous system (CNS). The disease involves most prominently the WM of the brain and the spinal cord with loss of myelin and axons. Histopathologic studies have demonstrated neocortical and neuronal pathology in MS and MRI studies have shown both lesions and diffuse GM changes in brains of MS patients. Measurements of the whole brain 
volume are sensitive to progression of the associated neuronal and axonal loss in MS patients across the different forms of the disease.

These measures can be related to clinical performance measures meaningful to patients. For example, balance and gait impairment are quantified using a combination of clinical examination and reported maximum walking distance. The Expanded Disability Status Scale (EDSS) ${ }^{2}$ is widely used as a measure of disability both in clinical practice and for clinical trials. However, it is a relatively subjective measure with high intra- and inter-ratter variability, so quantifying mild symptoms and symptom progression is challenging. Performance measures thus also have been used. For example, the Timed 25-Foot Walk (T25FW) measures the time a patient takes to walk 25 feet at maximum speed.

In this study we are going to compare both MT techniques when they are applied to the whole brain and WM of a cohort of $121 \mathrm{MS}$ patients. We will explore the similarity between MTR and MTsat measures across an MS population and we will analyse the capability to discriminate among the disability levels through the correlations with the EDSS and T25FW scores.

\subsection{Methods}

\section{Patients}

A hundred and twenty-one patients with rapidly evolving MS (REMS) were selected and enrolled in the Patient Research Cohort Rapidly Evolving Multiple Sclerosis study (PRC-REMS) (PI, Dr. Paolo Muraro) with the approval of the London-Chelsea Regional Ethics Committee. Their ages were between 25 until 66 years old with a diagnosis of MS according to the revised McDonald's criteria ${ }^{91}$ with relapsing- 
remitting MS (RRMS) or secondary progressive MS (SPMS), disease duration $\leq 15$ years from diagnosis and EDSS score (2.0-6.0) at screening.

\section{MRI acquisition}

The study was performed on a 3T whole body MR system (Magnetom Verio, Siemens Medical Solutions, Erlangen, Germany) equipped with 12-channel phased array head coil. A 3D FLASH sequence was performed with $1 \mathrm{~mm}$ of isotropic resolution, 192 sagittal partitions, 256*240 FOV and readout in the head-feet direction with 600 Hz/pixel bandwidth to reduce susceptibility artifacts. Six bipolar gradient echoes equally spaced between TE of 1.95 and $11.7 \mathrm{~ms}$ were acquired during a TR of $27 \mathrm{~ms}$. The measurement time was reduced to 7:20 min by means of partial Fourier in phase and slice directions and parallel acquisition (GRAPPA, iPAT factor 2). One PD-w dataset was acquired with a flip angle of $5^{\circ}$ and another PD-w dataset was MTweighting. The magnetization was saturated by a band selective RF pulse of Gaussian shape with a duration of $12.24 \mathrm{~ms}, 2.2 \mathrm{kHz}$ frequency offset and nominal flip angle $540^{\circ}$. A third dataset was acquired: one T1-w volume with a TR of $15 \mathrm{~ms}$ and a flip angle of $18^{\circ}$. All volumes were spatially co-registered to the MPRAGE dataset to correct any movement between the acquisitions.

\section{MTR maps}

The MTR maps were calculated as a percentage of signal loss:

$$
M T R=100\left(\text { MToff }_{P D w}-\text { MTon }_{P D w}\right) / \text { MToff } f_{P D w}
$$


Where $M T$ off $f_{P D w}$ is the signal intensity of the PD-w volume which was acquired without the saturation pulse and $M T o n_{P D w}$ is the signal intensity of the PD-w volume which was MT-weighted.

\section{MTsat maps}

The MTsat maps were calculated through a rational approximation of the Ernst equation, at short TR and small flip angle, for the FLASH signal where the second excitation is replaced by the MT pulse $\left(\text { MTon }_{\text {PDw }}\right)^{71,89}$. Two parameters should be calculated before which are the apparent proton density and the apparent $\mathrm{T} 1$ relaxation:

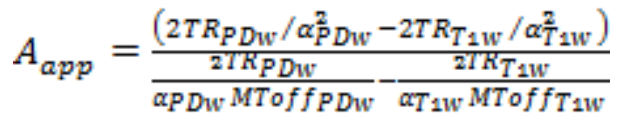

$$
\begin{aligned}
& T_{1 a p p}=\frac{\left(M T \circ f_{P D W} / \alpha_{P D w}-M T \circ f_{T 1 w} / \alpha_{T 1 w}\right)}{M T o f f_{P D w} \alpha_{P D w} / 2 T R_{P D w}}-M T o f f_{T 1 w} \alpha_{T 1 w} / 2 T R_{T 1 w}
\end{aligned}
$$

Where the TR's are in milliseconds and the flip angles $\alpha$ are in radians. MTsat can be calculated as follows:

$$
\text { MTsat }=100 A_{\text {app }} \alpha_{M T w} \frac{T R_{M T w}}{T_{1 a p p}} / \text { MTon }_{\text {PDw }}-\frac{T R_{M T w}}{T_{1 a p p}}-\frac{\alpha_{M T w}^{2}}{2}
$$

It is a dimensionless value and it is expressed in percent units (p.u.).

\section{Data Analysis}

An automated technique based on prior probabilities was used on T1-w images in the extraction of the brain and segmentation of GM and WM. The intensity distribution 
for the segmented MTR and MTsat maps was calculated by Matlab (Mathworks Natick, MA) producing the histograms. The whole brain, WM, GM histograms for both MTR and MTsat were calculated for each subject. They were divided into bins of width 0.25 p.u. and smoothed by convolution with a Gaussian kernel. To correct the difference of tissue volume between subjects, each histogram was normalized by dividing it by the total number of pixels.

Linear registration of datasets, brain extraction and arithmetic calculations were performed using (Flirt, Sienax, FSL, Centre for Functional Magnetic Resonance Imaging of the Brain, University of Oxford, UK).

WM lesions were marked and extracted manually with Analyze 11.0 (AnalyzeDirect/Mayo Clinic, KS). T2 hyperintensities of the FLAIR volumes were marked and co-registered in order to make the masks to get the NAWM.

Volumes were normalized with intracranial volume (ICV) to reduce interindividual variation and getting the white matter fraction $(\mathrm{WMF}=\mathrm{WM} / \mathrm{ICV})$, grey matter fraction $(\mathrm{GMF}=\mathrm{GM} / \mathrm{ICV})$, brain parenchyma fraction $(\mathrm{BPF}=(\mathrm{WM}+\mathrm{GM}) /$ ICV). The Bland-Altman test was used to assess the interchangeability between MTsat and MTR. Inter-subjects coefficients of variation $(\mathrm{CoV})$ were calculated:

$$
\mathrm{CoV}=\mathrm{SD} / \text { mean } \times 100 \%
$$

Spearman rank-order correlation coefficient and Spearman's partial correlations (controlling for age, gender and disease duration) assessed associations between WMF, GMF, BPF, T2LV volumes, MTR, MTsat, and both EDSS and T25FW. A p value (2-tailed) less than 0.05 was considered significant. Corrected thresholds for $p$ were applied for multiple comparisons. All statistical analyses were performed with SPSS for Windows (v. 17, SPSS Inc., Chicago, Illinois). 


\subsection{Results}

The study was performed for 121 patients with multiple sclerosis (115 RRMS and 6 SPMS). Their median (range) for the age was 39 (66-25) years, disease duration was 3 (16-0) years, EDSS score was 3.5 (6-2), T25FW was 5.9 (15.3-1.3) seconds and 2 (50) relapses within the year (Table 5.1).

Brain extraction and WM/GM/CSF segmentation were made on MToff-T1w images by an automated technique based on prior probabilities (Bet and Sienax, FSL) getting their masks which were visually inspected for assessing the quality of the segmentation. The MTsat maps showed a high T1-like contrast between WM, GM and CSF by comparison to the MTR maps (Figures 5.3 and 5.4) and were masked to get the whole brain, WM, GM and CSF regions. The average histograms for the whole brain and the WM/GM/CSF were calculated for the cohort of patients. The distribution of MT values across the brain showed three different modes for WM, GM and CSF (Figure 5.1). The MTR histogram did not show clear mode for the CSF. The WM/GM MTsat peaks had greater separation, reflecting higher contrast than the MTR (Figure 5.2). The histogram peak heights of the MTsat modes were correlated to the signal of the MTw image for each ROI. The peak height parenchyma MTsat was significantly correlated with the mean MTw (Spearman: $r=0.290 ; p=0.002$ ) and the MTR ( $\mathrm{r}=0.313 ; \mathrm{p}<0.001)$. The peak height of CSF MTsat was significantly correlated with the mean CSF MTw (Spearman: $r=0.658 ; \mathrm{p}<0.001$ ), but the peak height for CSF MTR ( $r=0.122 ; p=0.188)$. Overall, MTsat better discriminated between the brain tissue and the water than did MTR. The whole brain MTR and MTsat histograms were used to compare two clinical cases in Figure 5.4. Differences in their expression of the underlying pathology were apparent: the patient with the 
highest EDSS had a MTR histogram leak shifted towards the lower MT values, while the MTsat histogram showed an increase of the peak height CSF and a decrease for the WM.

The MTR and MTsat maps can be seen in Figure 5.3 for two clinical cases which had different T2 lesion volumes for different EDSS scores. The high score had a lesion volume higher than the patient with the low score. The MTsat maps showed higher contrast than the MTR for lesions and tissues.

Bland and Altman ${ }^{92,93}$ analysis was used to compare MTR and MTsat in which the difference versus the average of the median MTsat and MTR were compared in four regions of interest (ROI): whole brain, WM, normal appearing white matter (NAWM) and white matter lesions (WML) (Figure 5.7). In all cases, the bias were significantly different from zero confirming a mean difference between MTsat and MTR. The following limits of the agreement account for $95 \%$ of the samples between the following ranges of the difference (MTsat - MTR): $\pm 1.64 \%$ (whole brain), \pm $2.93 \%$ (WM), $\pm 2.93 \%$ (NAWM), and $\pm 3.44 \%$ (WM lesions).

Significant Spearman's correlations with EDSS were found for mean NAWM MTsat $(r=-0.215 ; p=0.018)$ and median NAWM MTsat $(r=-0.238 ; p=0.008)$. And significant Spearman correlations with T25FW for the same metrics: mean NAWM MTsat $(\mathrm{r}=-0.183 ; \mathrm{p}=0.045)$ and median NAWM MTsat $(\mathrm{r}=-0.180 ; \mathrm{p}=0.048)$ (Scatter plots in Figure 5.7). No significant correlations were found for mean and median NAWM MTR with neither EDSS nor T25FW (Tables 5.3 and 5.5). Spearman's partial correlation are shown in Tables 5.4 and 5.6. Scatter plots in Figure 5.6. The NAWM MTR and MTsat maps and histograms for two MS patients with EDSS scores of 2 and 6 are in Figure 5.5. It shows how both MTR histograms overlapped, while MTsat could "unfold" them. In the NAWM MTsat map, there are 
regions with lower MT values than the surrounding areas not found in the T2 FLAIR image.

Low intra-subject variation were found in the mean parameter values (2.74$8.10 \%$ ) for the mean/median whole brain, and mean/median NAWM in all the subjects for both techniques (Table 5.2).

\subsection{Discussion}

Several studies have shown that NAWM MTR values are altered in all of the major phenotypes of $\mathrm{MS}^{94}$. Reduced MTR values have been found in NAWM, even in patients with clinically definite MS and very few or no T2 visible lesions ${ }^{83}$. These observations have indicated the need to obtain more accurate estimates of the overall extent of NAWM damage in MS and, as a consequence, have led to the application of histogram analysis to all brain voxels classes as normal on cMRI (Figure 5.4). This study found significant Spearman's correlations for mean and median whole brain MTR and MTsat with EDSS. But no significant correlation for mean and median NAWM MTR and this could reflect lower sensitivity of the MTR to detect the subtle changes in the brain parenchyma. Mean and median NAWM MTsat are significantly correlated with both EDSS and T25FW, and this may reflect an improvement in the potential to detect changes. In fact, according to the pixel by pixel relaxation time analysis, the microscopic changes in the NAWM of MS do not occur randomly, but are related to the presence of small and discrete abnormalities, in some instances only one to two pixels in size ${ }^{69,95,96}$. MTsat is sensible to these subtle changes in the NAWM and this suggests that MTsat could be a better tool to analyse axonal loss and demyelination than MTR (Figure 5.5). 
Some studies found a correlation between T2 lesion volume in the brain and the EDSS, with the correlation coefficient generally in the order or $r=0.2$ in RRMS cohorts $^{59}$. In individuals, the relationship between lesion burden and disability can be very poor. Also, T2 lesion load measures do not take into consideration the contribution to disability of an abnormality in the NAWM. The lack of correlation between T2 lesion load and EDSS may be due to well recognized problems with the EDSS as a sensitive measure of neurological dysfunction and impairment. This study found a significant correlation between T2 lesion volume and EDSS $(r=0.254 ; \mathrm{p}=$ 0.005) and this suggests that it could be a measure of total nonspecific lesion pathology early in the course of relapsing remitting disease (Figure 5.3).

The results of Bland and Altman analysis show there is a mean value different from zero for the difference between MTR and MTsat in the four regions (whole brain, WM, NAWM and WML) (Figure 5.8). MTsat provides information distinct from that of the MT technique.

Several histogram parameters have been proposed in the literature and are correlated with the different clinical manifestations of $\mathrm{MS}^{84,90}$. The most frequently used parameters are peak height, peak location, mean and percentiles ${ }^{97}$. All of these parameters could be used to differentiate patients with MS from healthy controls ${ }^{90,98}$, different phenotypes of $\mathrm{MS}^{99}$, cortical atrophy ${ }^{100}$, changes in the WM and disability in the $\mathrm{GM}^{101,102}$, characterization of NAWM, normal appearing grey matter (NAGM) and diffuse appearing white matter (DAWM) ${ }^{103}$. Therefore, the peak height MTR represents burden of irreversible disease and the whole brain mean MTR is seen as a parameter which indicates the degree of tissue damage. The results presented here highlight that MTsat histogram is multimodal, while MTR histogram is unimodal; MTsat shows different modes related with the cerebrospinal fluid (CSF), GM and 
WM regions (Figure 5.1). Multimodal histograms cannot be well characterized by the unimodal features because the overlapping of the underlying broad structures prevents them being characterized independently of each other. Therefore, interpretations of MTR metrics (changes in peak height, shift of peak location, shift of mean and percentiles) may be different from those for MTsat, which may be able to be pathologically more specific.

\subsection{Conclusion}

MTsat and MTR can discriminate between disability levels in multiple sclerosis when the whole brain is analysed. In this study population, only MTsat displayed significant correlations for NAWM with EDSS and T25FW. MTsat could be show more sensitivity to the mild and subtle changes due to the disease in NAWM and provide a more clinically meaningful measure than MTR. 
Table 5.1: Summary of clinical characteristics of the patients involved in the study.

\begin{tabular}{|c|c|}
\hline Subjects & 121 \\
\hline Age (years) & $39(66-25)$ \\
\hline Gender (f/m) & $97 / 24$ \\
\hline Disease Duration (years) & $3(16-0)$ \\
\hline EDSS & $3.5(6-2)$ \\
\hline T25FW (seconds) & $5.9(15.3-1.3)$ \\
\hline RRMS & 115 \\
\hline SPMS & 6 \\
\hline Relapses within last year & $2(5-0)$ \\
\hline WMF & $0.37(0.02)$ \\
\hline GMF & $0.38(0.03)$ \\
\hline BPF & $0.75(0.03)$ \\
\hline T2LV (mL) & $19.4(12.9)$ \\
\hline
\end{tabular}

Median (range) values are shown for: age, disease duration, EDSS, T25FW and relapses within last year. Mean (standard deviation) values are for: WMF, GMF, BPF and $\mathrm{T} 2 \mathrm{LV}$.

Abbreviations: EDSS = Expanded Disability Status Scale, T25FW = timed 25-foot walk, RRMS = relapsing remitting multiple sclerosis, SPMS = secondary progressive multiple sclerosis, $\mathrm{WMF}=$ white matter fraction, $\mathrm{GMF}=$ grey matter fraction, $\mathrm{BPF}=$ brain parenchyma fraction, $\mathrm{T} 2 \mathrm{LV}=\mathrm{T} 2$ lesion volume.

Table 5.2: MTR and MTsat metrics. Mean, median and peak location are in p.u. Peak height is expressed by normalized number of pixels. CoVs are in p.u.

\begin{tabular}{|c|c|c|c|c|}
\hline & MTR & CoV & MTsat & CoV \\
\hline & & & & \\
\hline Mean whole & $38.71(1.64)$ & 4.24 & $16.83(1.31)$ & 7.78 \\
\hline Mean NAWM & $47.91(1.38)$ & 2.88 & $26.04(1.96)$ & 7.53 \\
\hline Mean WML & $44.92(2.35)$ & 5.23 & $20.61(2.80)$ & 13.59 \\
\hline & & & & \\
\hline Median whole & $42.36(1.16)$ & 2.74 & $15.79(1.00)$ & 6.33 \\
\hline Median NAWM & $48.46(1.35)$ & 2.79 & $26.43(2.13)$ & 8.10 \\
\hline Median WML & $46.64(2.36)$ & 5.06 & $21.58(3.30)$ & 15.29 \\
\hline & & & & \\
\hline Peak location whole & $47.28(2.26)$ & 4.78 & $12.25(4.35)$ & 35.51 \\
\hline Peak location WML & $48.92(2.05)$ & 4.19 & $24.93(3.15)$ & 12.64 \\
\hline & & & & \\
\hline Peak height whole & $0.018(0.001)$ & 5.55 & $0.018(0.002)$ & 11.11 \\
\hline Peak height WML & $0.044(0.006)$ & 13.64 & $0.032(0.005)$ & 15.63 \\
\hline
\end{tabular}


Abbreviations: MTR $=$ magnetization transfer ratio, MTsat $=$ magnetization transfer saturation, $\mathrm{CoV}=$ coefficient of variation, $\mathrm{NAWM}=$ normal appearing white matter, $\mathrm{WML}=$ white matter lesions.

Table 5.3: Spearman rank-order correlation coefficients with EDSS for MTR and MTsat (*p $<0.05$, corrected for multiple comparisons).

\begin{tabular}{|c|c|c|}
\hline & \multicolumn{2}{|c|}{ EDSS } \\
\hline MTR & $\mathrm{r}$ & $\mathrm{p}$ Value \\
\hline Mean whole brain & -0.277 & $0.002 *$ \\
\hline Mean NAWM & -0.110 & 0.228 \\
\hline Mean WM lesions & -0.267 & $0.003 *$ \\
\hline Median whole brain & -0.271 & $0.003 *$ \\
\hline Median NAWM & -0.178 & 0.051 \\
\hline Median WM lesions & -0.264 & $0.003 *$ \\
\hline Peak height whole brain & 0.033 & 0.719 \\
\hline Peak height NAWM & -0.042 & 0.646 \\
\hline Peak height WM lesions & -0.101 & 0.273 \\
\hline MTsat & $\mathrm{r}$ & $\mathrm{p}$ Value \\
\hline Mean whole brain & -0.282 & $0.002 *$ \\
\hline Mean NAWM & -0.215 & $0.018^{*}$ \\
\hline Mean WM lesions & -0.230 & $0.011^{*}$ \\
\hline Median whole brain & -0.288 & $0.001 *$ \\
\hline Median NAWM & -0.238 & $0.008^{*}$ \\
\hline Median WM lesions & -0.230 & $0.011 *$ \\
\hline Peak height whole brain & 0.014 & 0.882 \\
\hline Peak height NAWM & -0.011 & 0.905 \\
\hline Peak height WM lesions & -0.017 & 0.852 \\
\hline
\end{tabular}

Abbreviations: MTR $=$ magnetization transfer ratio, MTsat $=$ magnetization transfer saturation, EDSS = Expanded Disability Status Scale, NAWM = normal appearing white matter, $\mathrm{WM}=$ white matter. 
Table 5.4: Spearman's partial correlations (control variables) with EDSS for MTR and MTsat $(* * \mathrm{p}<0.017$ and $* * * \mathrm{p}<0.005$; Bonferroni corrected $\mathrm{p}$ value thresholds)

\begin{tabular}{|c|c|c|c|c|}
\hline \multirow[b]{3}{*}{ MTR } & \multicolumn{4}{|c|}{ EDSS } \\
\hline & \multicolumn{2}{|c|}{ Partial correlation (age) } & \multicolumn{2}{|c|}{$\begin{array}{l}\text { Partial correlation (age, } \\
\text { gender ,disease duration) }\end{array}$} \\
\hline & $\mathrm{r}$ & $\mathrm{p}$ Value & $r$ & $\mathrm{p}$ Value \\
\hline Mean whole brain & -0.214 & 0.019 & -0.197 & 0.033 \\
\hline Mean NAWM & -0.099 & 0.283 & -0.064 & 0.493 \\
\hline Mean WM lesions & -0.262 & $0.004 * *$ & -0.254 & 0.005 \\
\hline Median whole brain & -0.240 & $0.008 * *$ & -0.211 & 0.022 \\
\hline Median NAWM & -0.137 & 0.135 & -0.102 & 0.274 \\
\hline Median WM lesions & -0.271 & $0.003 * *$ & -0.256 & 0.005 \\
\hline Peak height whole brain & 0.022 & 0.816 & -0.015 & 0.872 \\
\hline Peak height NAWM & -0.001 & 0.987 & -0.020 & 0.832 \\
\hline Peak height WM lesions & -0.093 & 0.313 & -0.131 & 0.158 \\
\hline MTsat & $\mathrm{r}$ & $\mathrm{p}$ Value & $\mathrm{r}$ & $\mathrm{p}$ Value \\
\hline Mean whole brain & -0.252 & $0.006 * *$ & -0.266 & $0.004 * * *$ \\
\hline Mean NAWM & -0.200 & 0.028 & -0.214 & 0.020 \\
\hline Mean WM lesions & -0.243 & $0.007 * *$ & -0.270 & $0.003 * * *$ \\
\hline Median whole brain & -0.266 & $0.003 * *$ & -0.286 & $0.002 * * *$ \\
\hline Median NAWM & -0.223 & $0.014 * *$ & -0.243 & 0.007 \\
\hline Median WM lesions & -0.251 & $0.006 * *$ & -0.271 & $0.003 * * *$ \\
\hline Peak height whole brain & 0.047 & 0.608 & 0.054 & 0.563 \\
\hline Peak height NAWM & -0.062 & 0.499 & -0.063 & 0.497 \\
\hline Peak height WM lesions & -0.040 & 0.664 & -0.028 & 0.762 \\
\hline
\end{tabular}

Abbreviations: MTR $=$ magnetization transfer ratio, MTsat $=$ magnetization transfer saturation, EDSS $=$ Expanded Disability Status Scale, NAWM = normal appearing white matter, $\mathrm{WM}=$ white matter. 
Table 5.5: Spearman rank-order correlation coefficients with T25FW for MTR and MTsat $(* p<0.05$, corrected for multiple comparisons)

\begin{tabular}{|c|c|c|}
\hline & \multicolumn{2}{|c|}{ T25FW } \\
\hline MTR & $\mathrm{r}$ & $\mathrm{p}$ Value \\
\hline Mean whole brain & -0.222 & $0.014^{*}$ \\
\hline Mean NAWM & -0.106 & 0.247 \\
\hline Mean WM lesions & -0.274 & $0.002^{*}$ \\
\hline & & \\
\hline Median whole brain & -0.263 & $0.004^{*}$ \\
\hline Median NAWM & -0.170 & 0.062 \\
\hline Median WM lesions & -0.328 & $<0.001^{*}$ \\
\hline & & \\
\hline Peak height whole brain & 0.022 & 0.807 \\
\hline Peak height NAWM & -0.118 & 0.196 \\
\hline Peak height WM lesions & -0.209 & $0.021^{*}$ \\
\hline & & \\
\hline MTsat & $\mathrm{r}$ & $\mathrm{p} \mathrm{Value}$ \\
\hline & & \\
\hline Mean whole brain & -0.249 & $0.006^{*}$ \\
\hline Mean NAWM & -0.183 & $0.045^{*}$ \\
\hline Mean WM lesions & -0.219 & $0.016^{*}$ \\
\hline Median whole brain & -0.225 & $0.013^{*}$ \\
\hline Median NAWM & -0.180 & $0.048^{*}$ \\
\hline Median WM lesions & -0.251 & $0.005^{*}$ \\
\hline Peak height whole brain & 0.140 & 0.125 \\
\hline Peak height NAWM & -0.004 & 0.964 \\
\hline & -0.014 & 0.878 \\
\hline
\end{tabular}

Abbreviations: MTR $=$ magnetization transfer ratio, MTsat $=$ magnetization transfer saturation, $\mathrm{T} 25 \mathrm{FW}=$ timed 25 -foot walk, NAWM = normal appearing white matter, $\mathrm{WM}=$ white matter . 
Table 5.6: Spearman's partial correlations (control variables) with T25FW for MTR and MTsat $(* * \mathrm{p}<0.017$ and $* * * \mathrm{p}<0.005 ;$ Bonferroni corrected $\mathrm{p}$ value thresholds)

\begin{tabular}{|c|c|c|c|c|}
\hline & \multicolumn{4}{|c|}{ T25FW } \\
\hline & \multicolumn{2}{|c|}{ Partial correlation (age) } & \multicolumn{2}{|c|}{$\begin{array}{l}\text { Partial correlation (age, } \\
\text { gender, disease duration) }\end{array}$} \\
\hline MTR & $\mathrm{r}$ & $\mathrm{p}$ Value & $\mathrm{r}$ & $\mathrm{p}$ Value \\
\hline Mean whole brain & -0.189 & 0.039 & -0.197 & 0.032 \\
\hline Mean NAWM & -0.099 & 0.284 & -0.093 & 0.316 \\
\hline Mean WM lesions & -0.268 & $0.003 * *$ & -0.265 & $0.004 * * *$ \\
\hline Median whole brain & -0.244 & $0.007 * *$ & -0.237 & 0.010 \\
\hline Median NAWM & -0.148 & 0.106 & -0.141 & 0.128 \\
\hline Median WM lesions & -0.329 & $<0.001 * *$ & -0.320 & $<0.001 * * *$ \\
\hline Peak height whole brain & 0.016 & 0.860 & -0.015 & 0.871 \\
\hline Peak height NAWM & -0.100 & 0.278 & -0.129 & 0.165 \\
\hline Peak height WM lesions & -0.206 & 0.024 & -0.235 & 0.010 \\
\hline MTsat & $\mathrm{r}$ & $\mathrm{p}$ Value & $\mathrm{r}$ & $\mathrm{p}$ Value \\
\hline Mean whole brain & -0.230 & $0.011 * *$ & -0.255 & 0.005 \\
\hline Mean NAWM & -0.172 & 0.061 & -0.193 & 0.037 \\
\hline Mean WM lesions & -0.222 & $0.015 * *$ & -0.246 & 0.007 \\
\hline Median whole brain & -0.210 & 0.021 & -0.237 & 0.010 \\
\hline Median NAWM & -0.168 & 0.066 & -0.193 & 0.035 \\
\hline Median WM lesions & -0.258 & $0.004 * *$ & -0.276 & $0.002 * * *$ \\
\hline Peak height whole brain & 0.159 & 0.083 & -0.155 & 0.094 \\
\hline Peak height NAWM & -0.029 & 0.754 & -0.048 & 0.605 \\
\hline Peak height WM lesions & -0.025 & 0.786 & -0.023 & 0.804 \\
\hline
\end{tabular}

Abbreviations: MTR $=$ magnetization transfer ratio, MTsat $=$ magnetization transfer saturation, $\mathrm{T} 25 \mathrm{FW}=$ timed 25 -foot walk, NAWM = normal appearing white matter, $\mathrm{WM}=$ white matter. 
Figure 5.1: Average whole brain and tissue compartments MTR and MTsat histograms of all the subjects. The MTR histogram is unimodal meanwhile the MTsat is multimodal. Brain extraction and WM, GM and CSF were estimated with SienaxFSL which extracted brain and skull images from the input data including separated volumes for WM, GM and CSF. The whole brain mask was used to make the whole brain histograms after masking the MTR and MTsat maps. The WM, GM and CSF were masked respectively and their histograms calculated and finally averaged for the whole cohort.
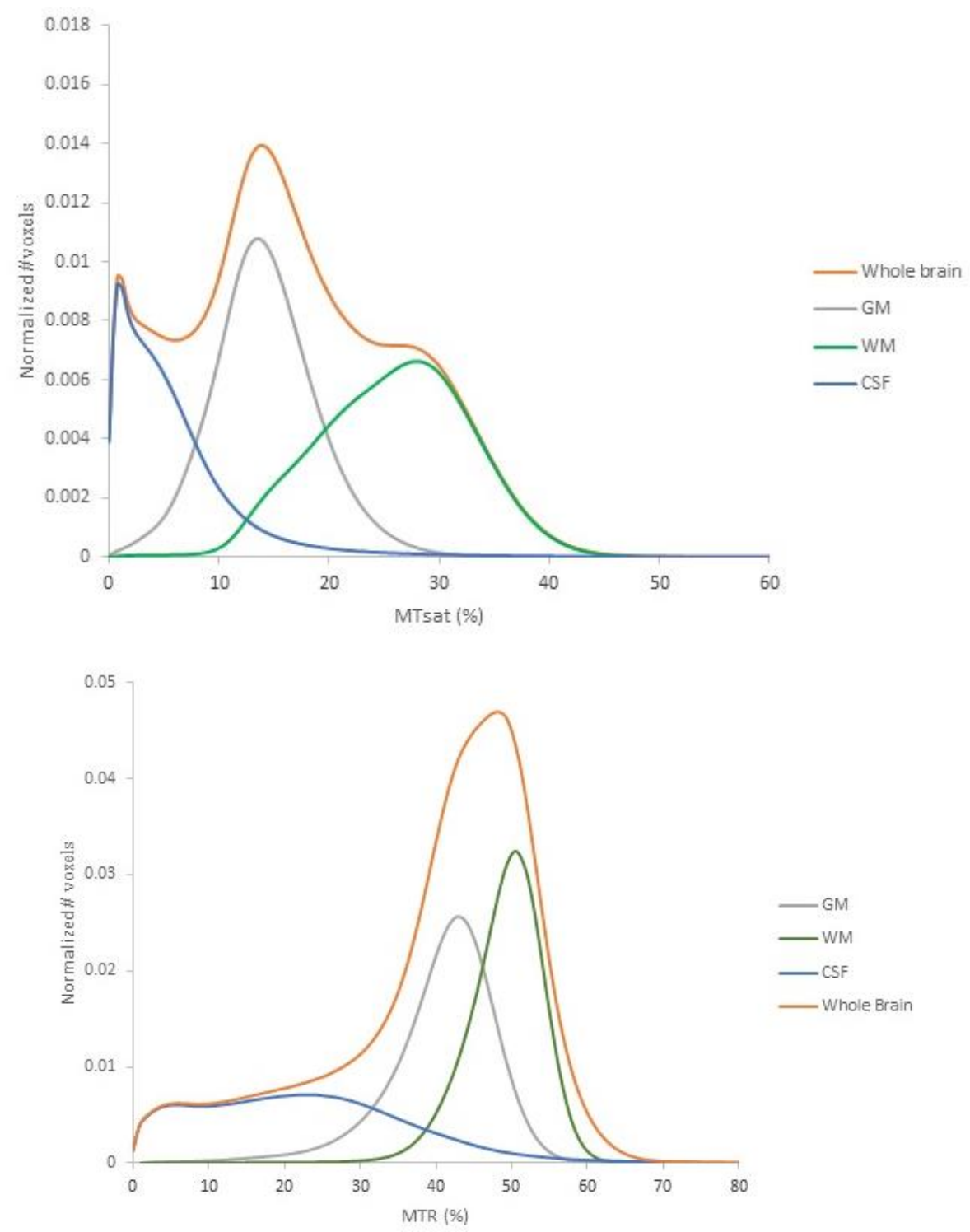

Abbreviations: MTR $=$ magnetization transfer ratio, MTsat $=$ magnetization transfer saturation, $\mathrm{WM}=$ white matter, $\mathrm{GM}=$ grey matter, $\mathrm{CSF}=$ cerebrospinal fluid. 
Figure 5.2: Average MTsat and MTR histograms of the GM and WM.

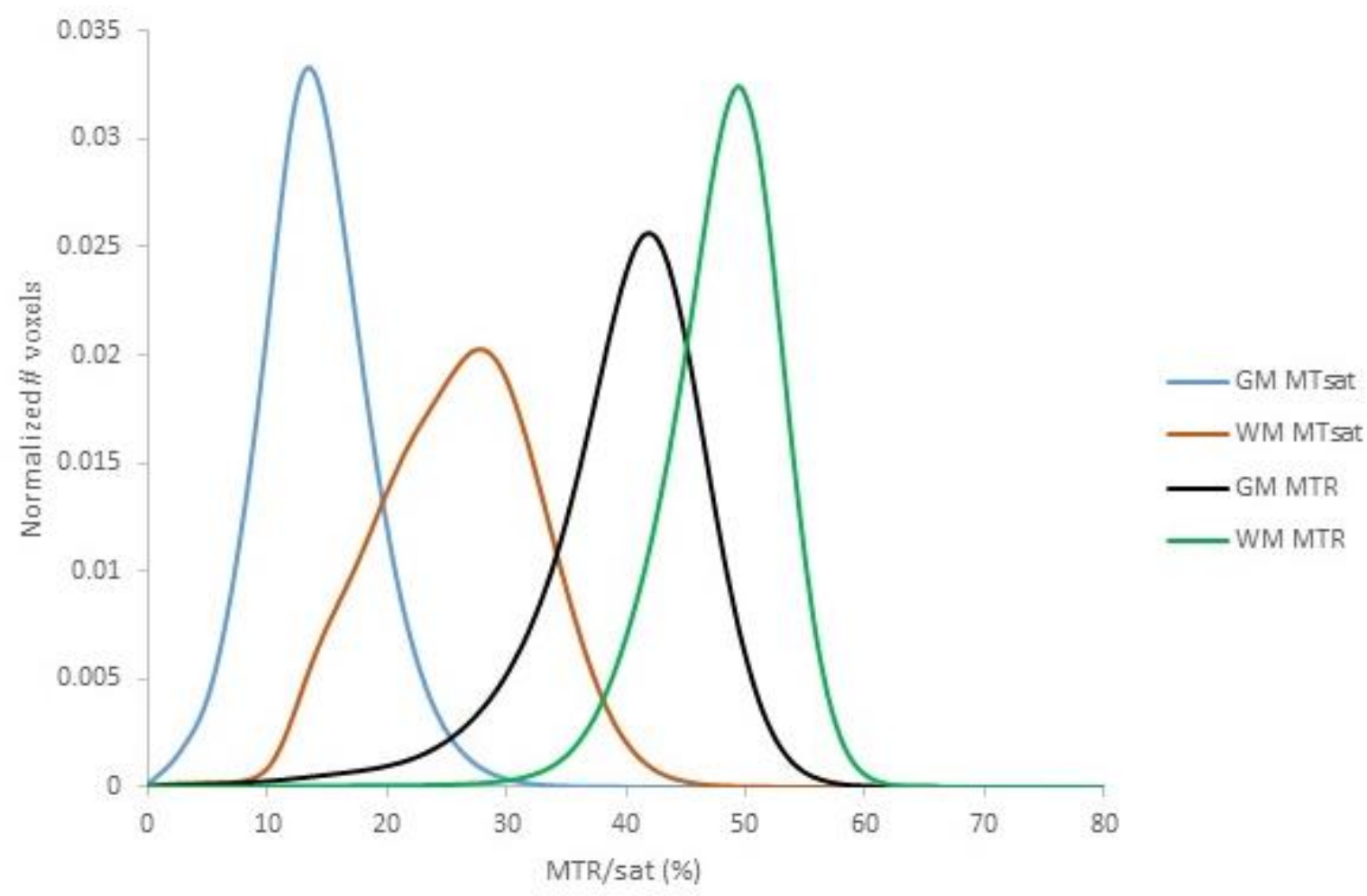

Abbreviations: MTR $=$ magnetization transfer ratio, MTsat $=$ magnetization transfer saturation, $\mathrm{WM}=$ white matter, $\mathrm{GM}=$ grey matter, $\mathrm{CSF}=$ cerebrospinal fluid.

Figure 5.3: Brain MT maps from two MS patients (Study codes Patient A: CRT114127_000037; Patient B: CRT1141127_000040). The top two rows (Patient A) are MTR (first row) and MTsat (second row) maps samples from a 55 years old woman with a history of RRMS with an EDSS score of 6 and a T25FW of 12.9 sec. The patient shows a total brain lesion volume of $43.56 \mathrm{~mL}$ The bottom two rows (Patient B) are MTR (first row) and MTsat (second row) maps samples from a 34 years old woman with a history of RRMS with an EDSS score of 2 and T25FW score of $6 \mathrm{sec}$. In this case the total brain volume lesion is $12.13 \mathrm{~mL}$. 


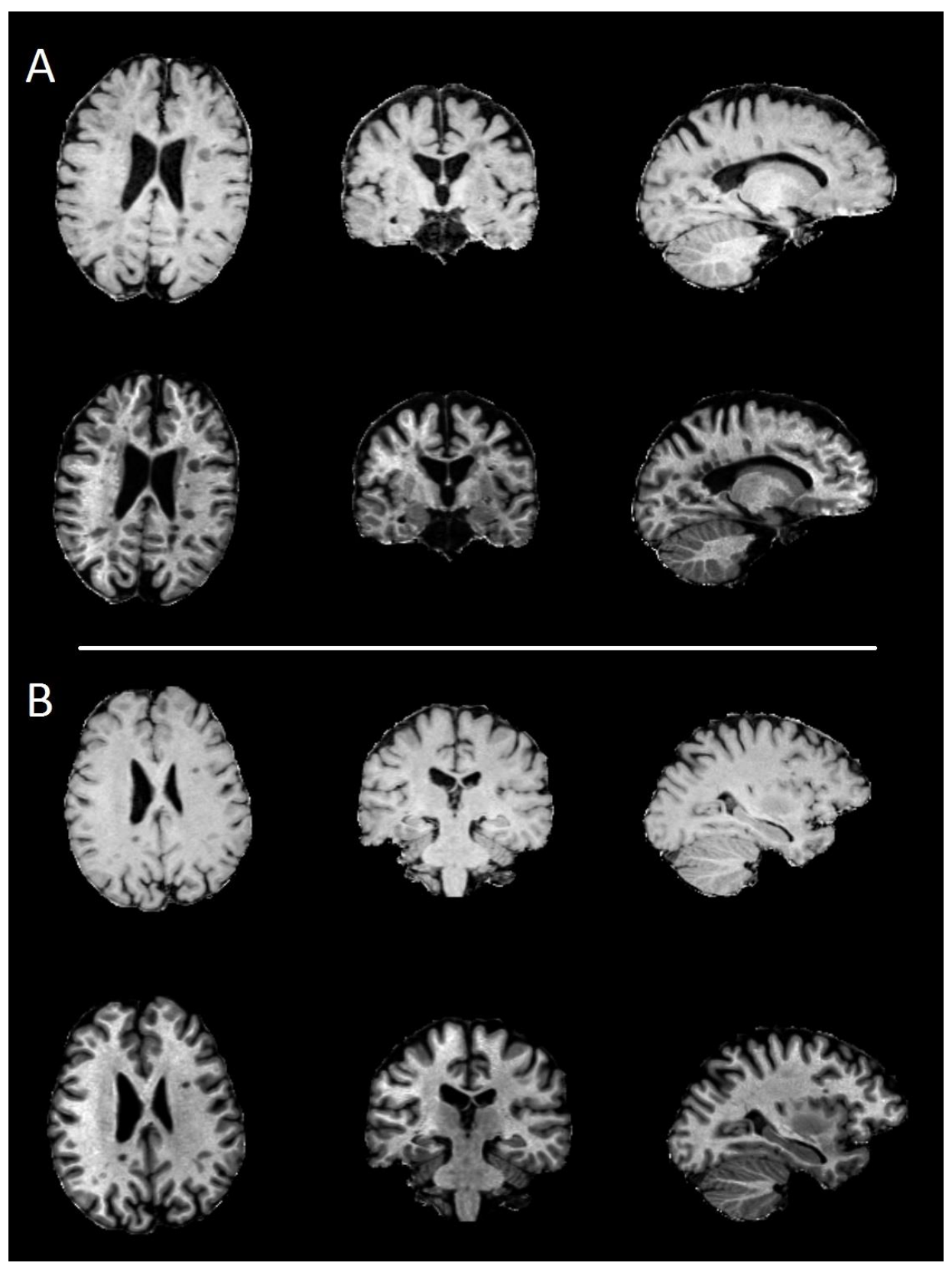

Abbreviations: $\mathrm{MT}=$ magnetization transfer, $\mathrm{MS}=$ multiple sclerosis, $\mathrm{MTR}=$ magnetization transfer ratio, MTsat $=$ magnetization transfer saturation, $\mathrm{RRMS}=$ relapsing remitting multiple sclerosis, EDSS = Expanded Disability Status Scale, $\mathrm{T} 25 \mathrm{FW}=$ timed 25-foot walk.

Figure 5.4: Axial MTR and MTsat maps and whole brain MTR and MTsat histograms of two patients (Study codes Patient A: CRT114127_000037; Patient B: CRT1141127_000088). Patient A is a 55 years old woman with a history of RRMS 
with an EDSS score of 6 and a T25FW of 12.9 sec. Patient B is a 40 years old man with a history of RRMS with an EDSS score of 2 and a T25FW of 4 sec. Differences in the MTR and MTsat histograms show how both MT techniques are able to reflect the burden of disease. The MTR histograms of the more disabled patient shows a histogram with a lower peak height and increased CSF area than the less disabled. MTsat histograms show changes in the CSF and WM modes. There is an increase in the peak height of the CSF region and decreased peak height WM for the patient A.
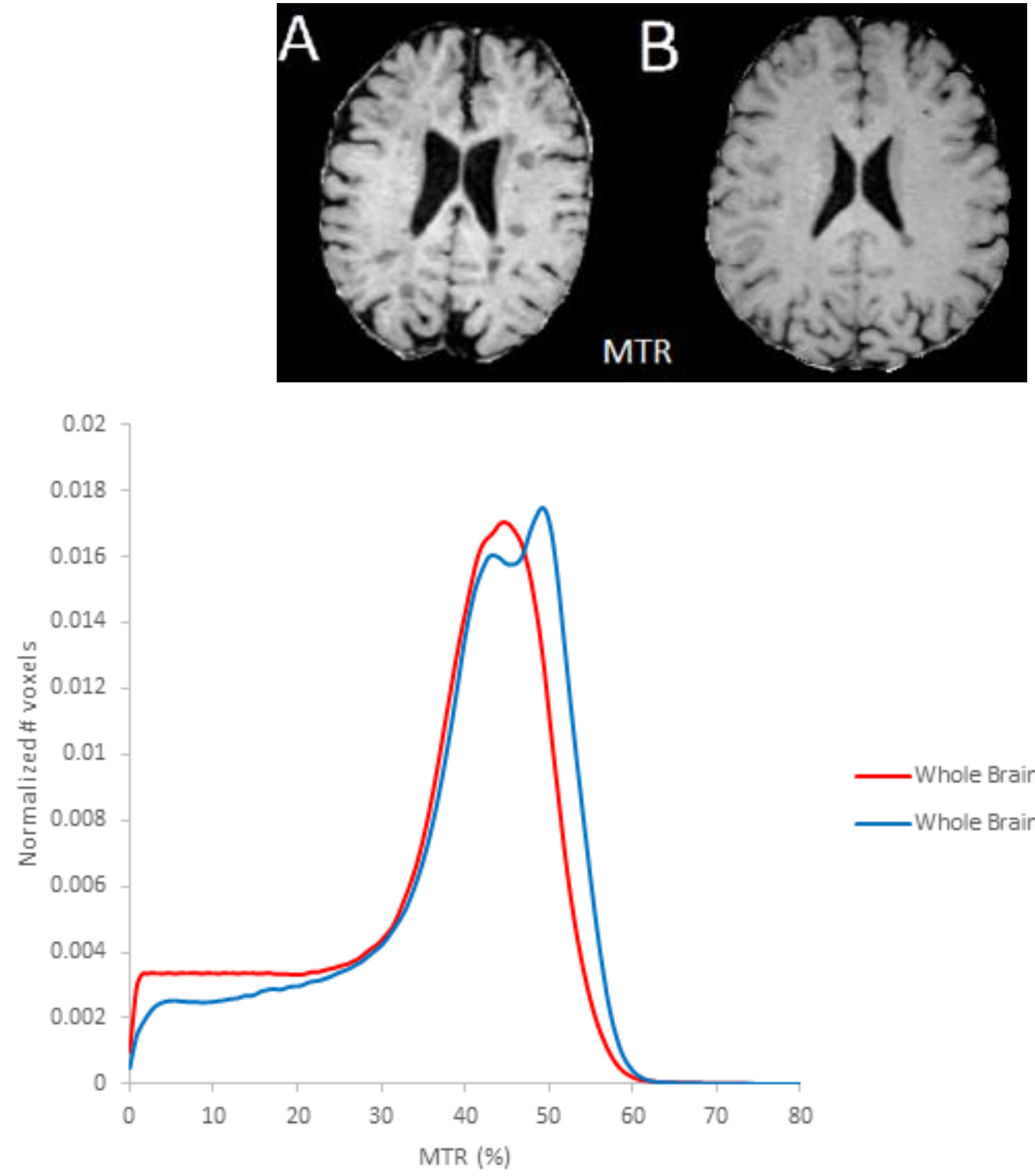

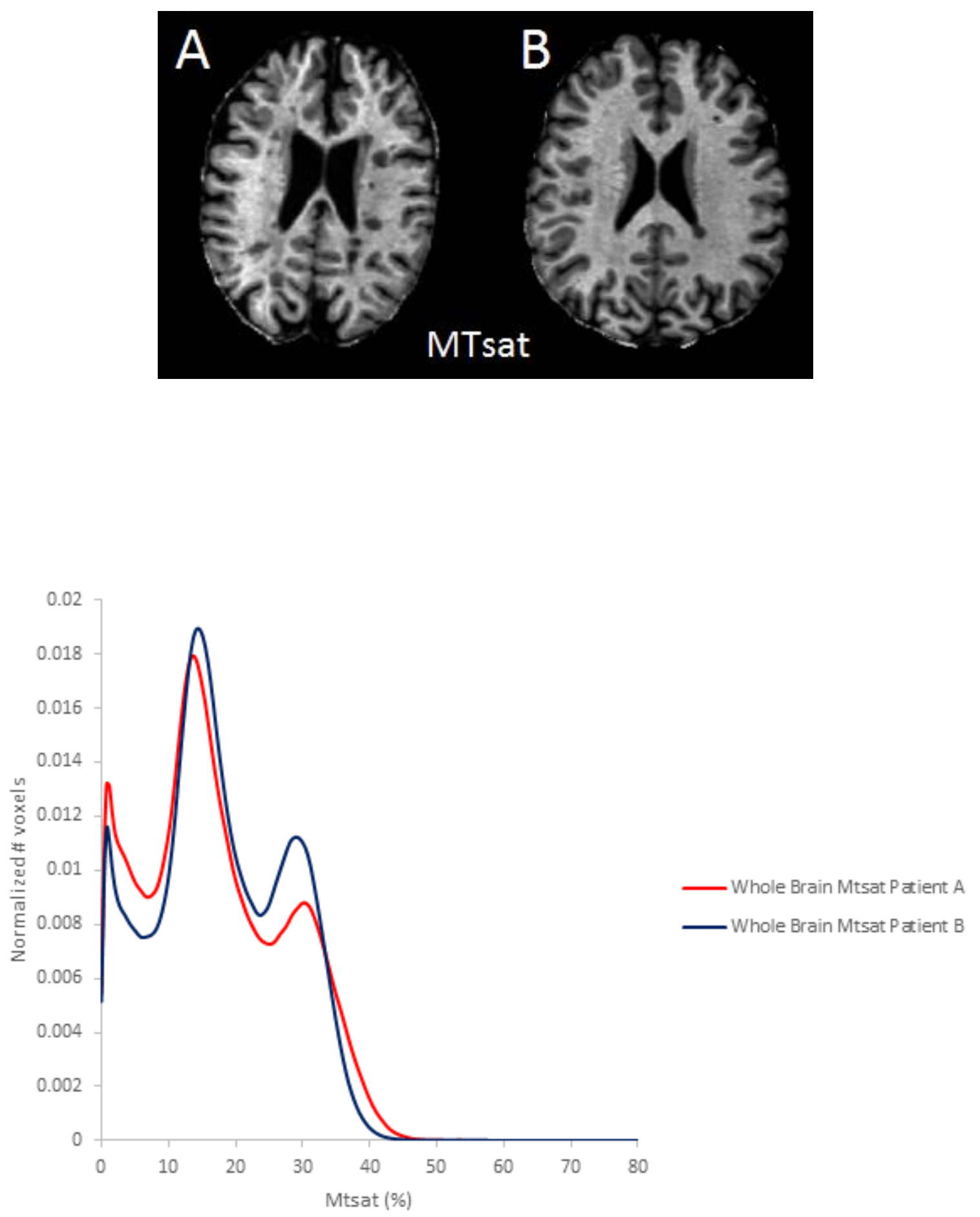

Abbreviations: MTR $=$ magnetization transfer ratio, MTsat $=$ magnetization transfer saturation, RRMS = relapsing remitting multiple sclerosis, EDSS = Expanded Disability Status Scale, $\mathrm{T} 25 \mathrm{FW}=$ timed 25 -foot walk, $\mathrm{WM}=$ white matter, $\mathrm{CSF}=$ cerebrospinal fluid. 
Figure 5.5: Axial NAWM MTR and MTsat of two patients (Study codes Patient A: GSK111009_000159; Patient B: GSK111009_000166) Patient A is a 48 years old woman with a history of RRMS with an EDSS score of 6 and a T25FW of $14.1 \mathrm{sec}$. The patient shows a total brain lesion volume of $19.62 \mathrm{~mL}$. Patient B is a 38 years old man with a history of RRMS with an EDSS score of 2 and T25FW score of $5.4 \mathrm{sec}$. In this case the total brain volume lesion is $1.80 \mathrm{~mL}$. NAWM MTsat is able to discriminate between these two different levels of disability meanwhile NAWM MTR histograms overlap.
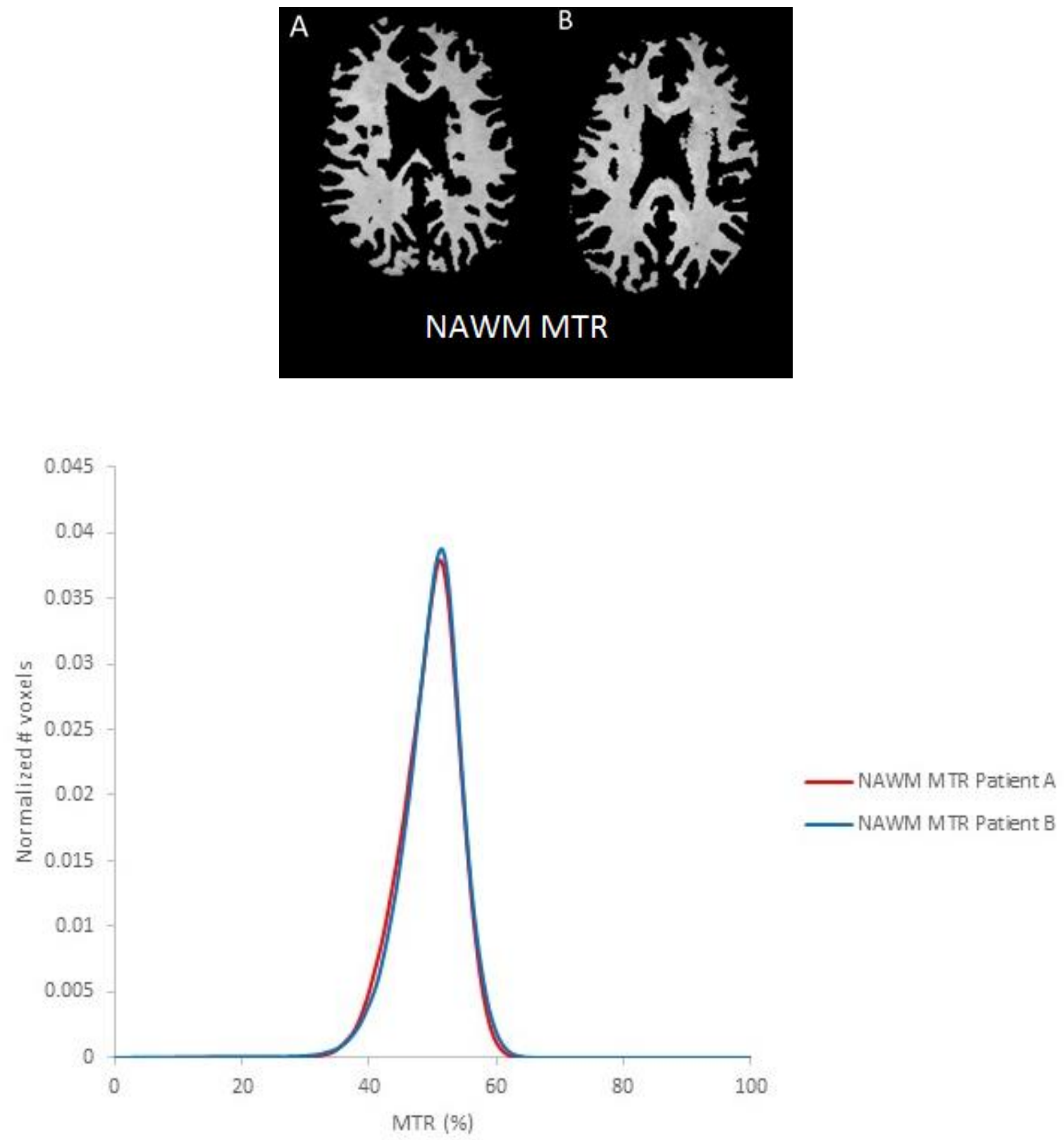

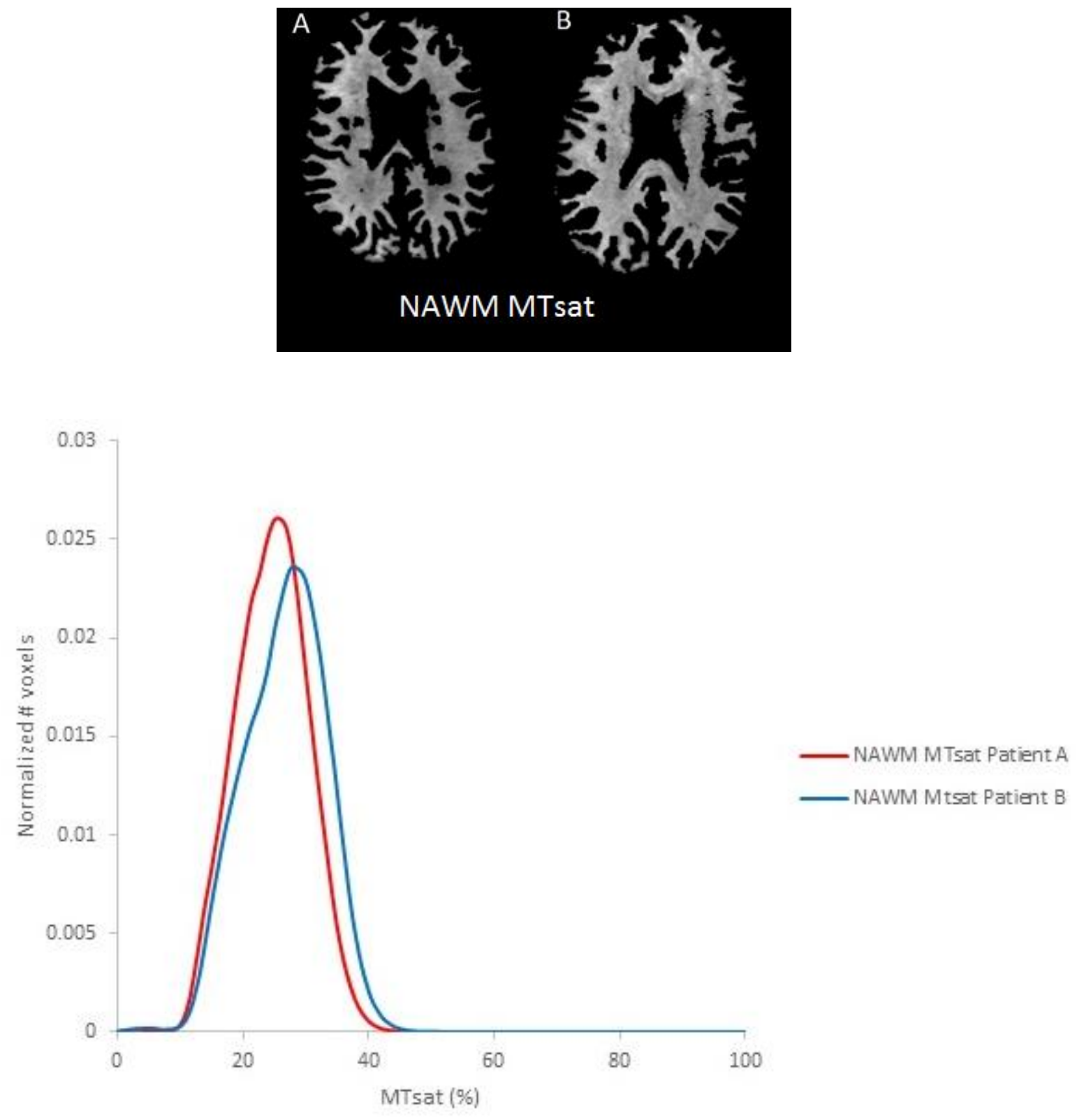

Abbreviations: NAWM = normal appearing white matter, $\mathrm{MTR}=$ magnetization transfer ratio, MTsat $=$ magnetization transfer saturation, $\mathrm{RRMS}=$ relapsing remitting multiple sclerosis, EDSS = Expanded Disability Status Scale, T25FW = timed 25-foot walk. 
Figure 5.6: Scatter plots of median NAWM MTR and median NAWM MTsat against EDSS and T25FW
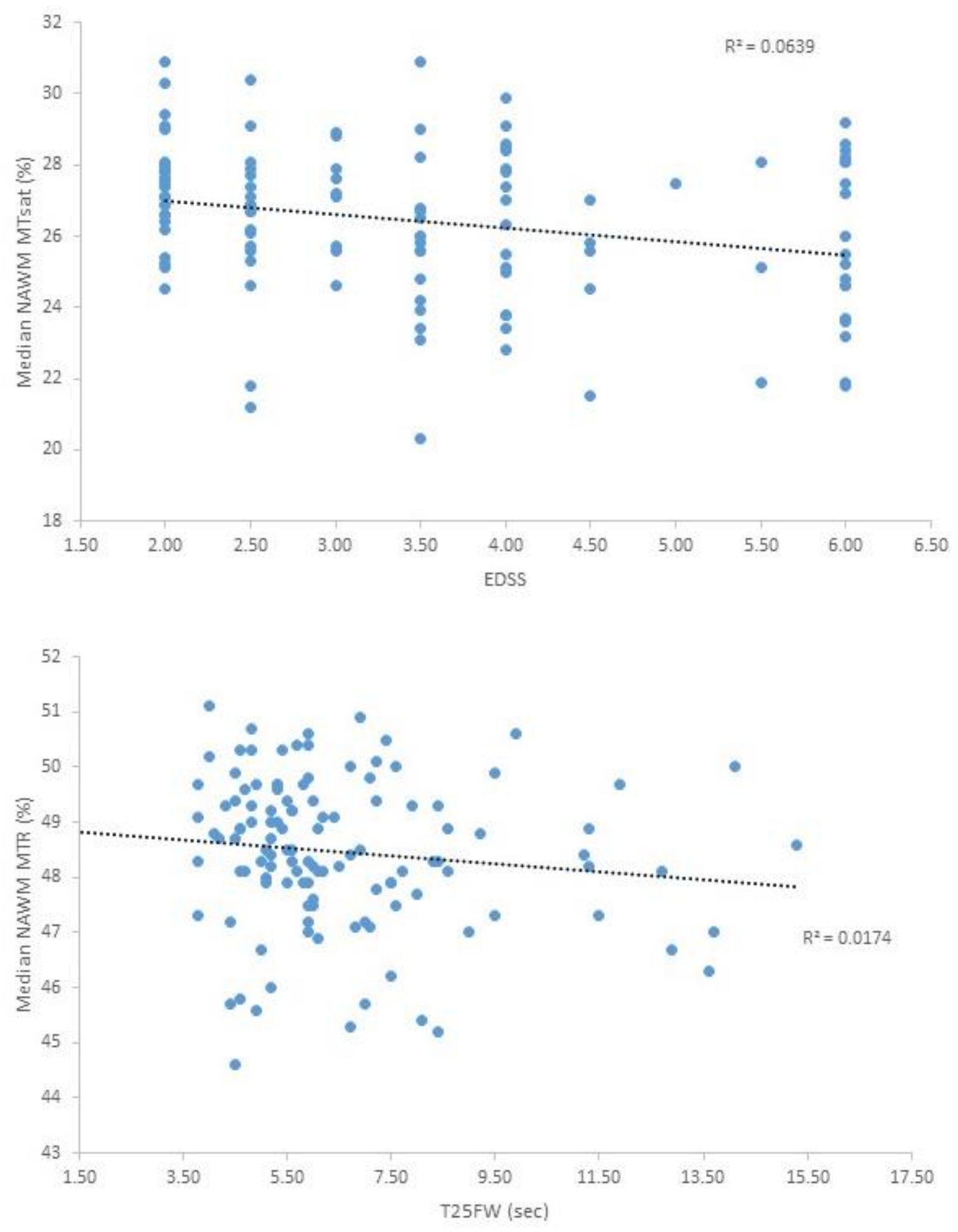


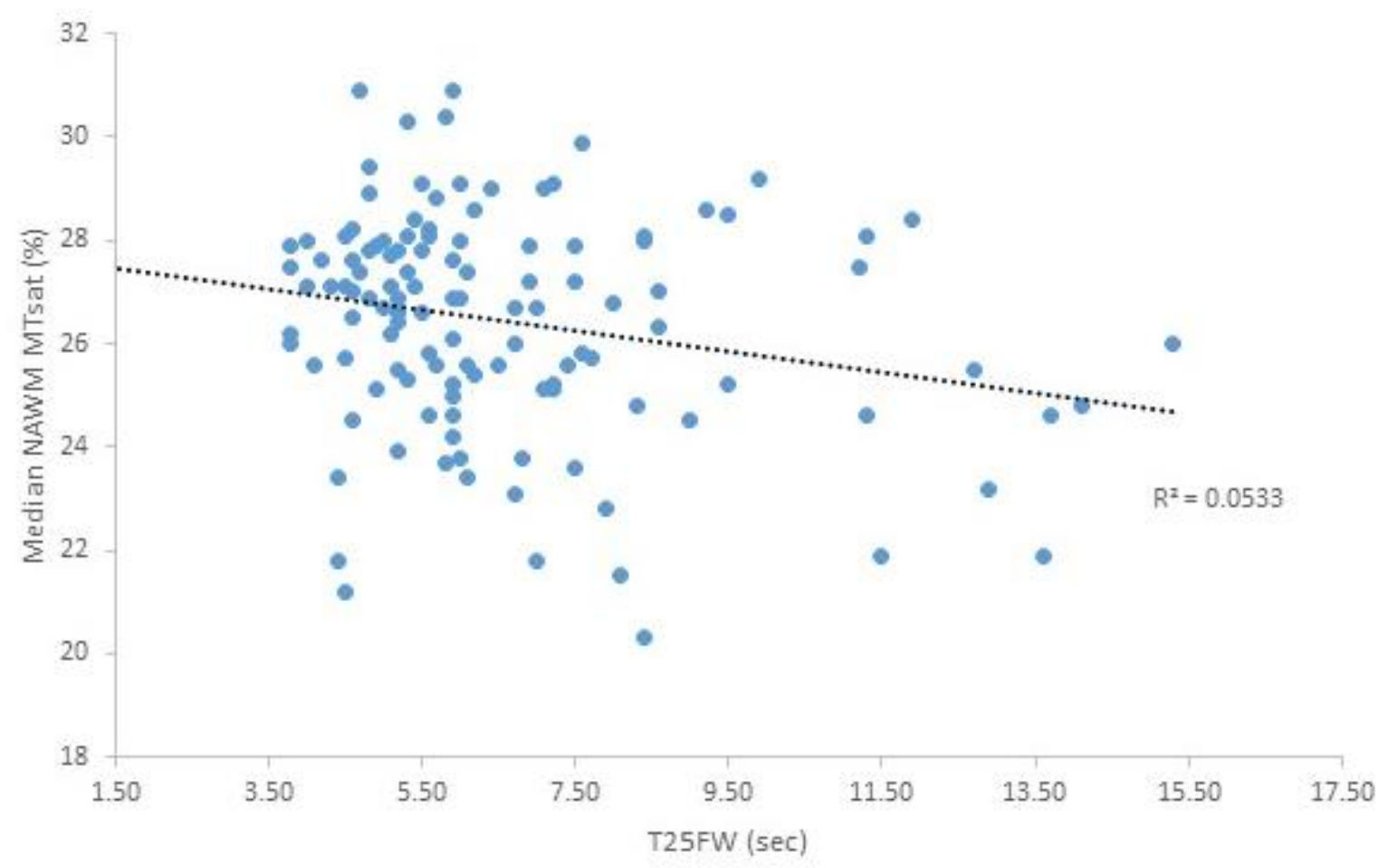

Abbreviations: NAWM $=$ normal appearing white matter, $\mathrm{MTR}=$ magnetization transfer ratio, MTsat $=$ magnetization transfer saturation, EDSS $=$ Expanded Disability Status Scale, T25FW = timed 25-foot walk. 
Figure 5.7: Bland-Altman plots for the whole brain, WM, NAWM and WM lesions. The green line shows the mean of the difference and the red lines are the limits of agreement (mean $\pm 1.96 \mathrm{SD}$ ). There were a significant linear regression for the WM, NAWM and WM lesions, but not for the whole brain. This suggests a proportional error or skewed samples.

\section{Whole Brain}

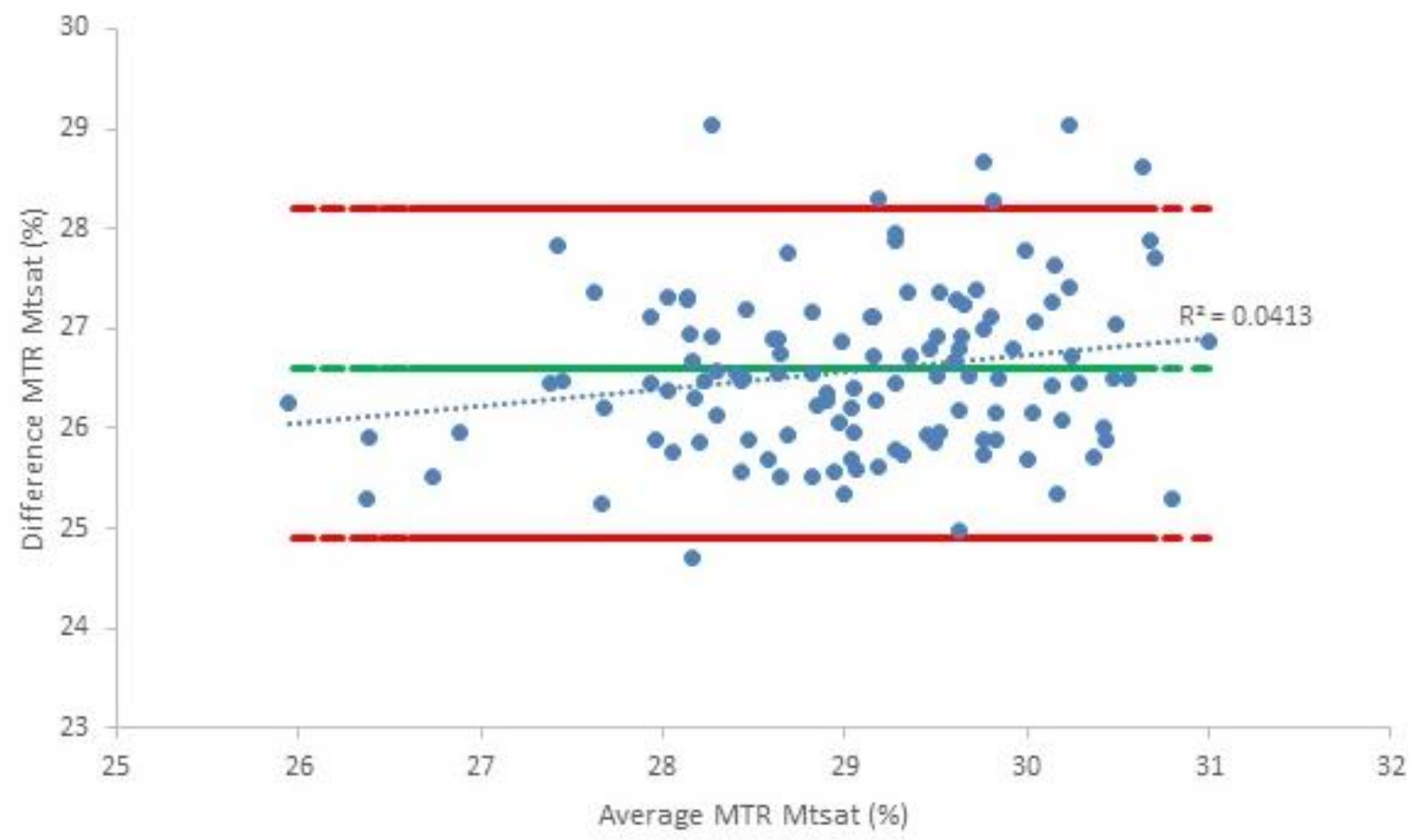



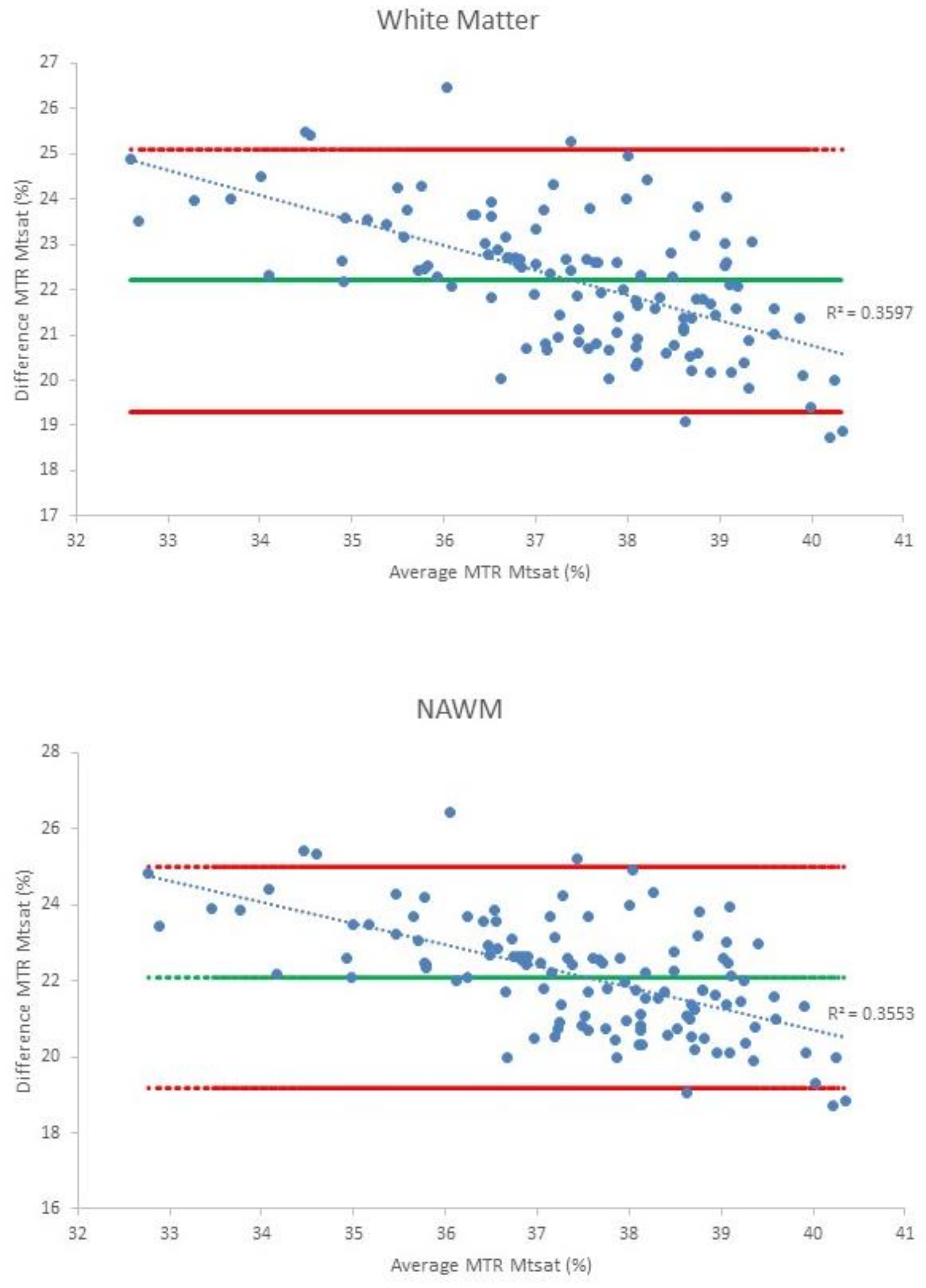


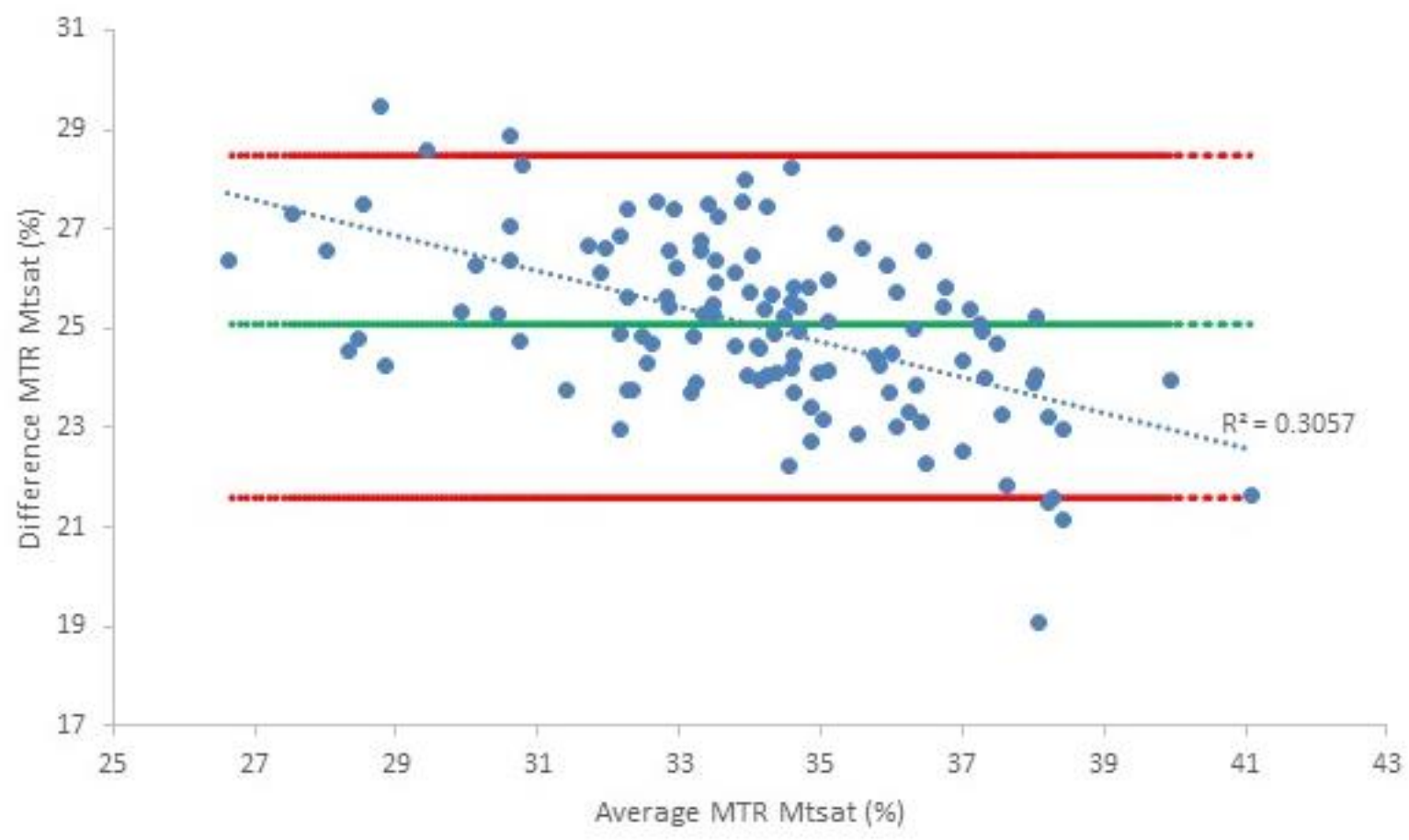

Abbreviations: $\mathrm{WM}=$ white matter, $\mathrm{NAWM}=$ normal appearing white matter, $\mathrm{WML}$ $=$ white matter lesions, MTR $=$ magnetization transfer ratio, MTsat $=$ magnetization transfer saturation. 


\section{Chapter 6}

\section{Cervical cord high resolution MT maps}

\subsection{Introduction}

Multiple sclerosis (MS) is an inflammatory demyelinating disorder of the central nervous system (CNS). The disease involves most prominently the white matter (WM) of the brain and the spinal cord (SC) with loss of myelin and axons. The relationship between the location of the lesions in the brain and the clinically overt symptoms is not always clear. It seems plausible that those in the spinal cord (SC) could have a stronger relationship, but they are more difficult to quantify. Cord atrophy correlates strongly with disability, for example ${ }^{104}$. It is known that clinically silent lesions are found in the SC as well and the understanding of their role in the course of the disease will help us to know the disability properly ${ }^{105}$. Normal appearing brain matter (NABM) refers to the regions without focal lesions on T2 weighted images. However, histopathology post-mortem has revealed subtle changes outside of lesions and it is microscopically abnormal ${ }^{106,107}$. Quantitative MRI techniques can demonstrate similar abnormalities in vivo ${ }^{69,96}$. The normal appearing cord tissue shows diffuse damage which is also relevant and occurs independently of brain pathology $\mathrm{y}^{108}$. 
One of the most promising techniques for characterising the diffuse and microstructural damage is the magnetization transfer ratio (MTR). Significant correlations were found with MTR and disability in the C3/4 level of the cervical cord $^{109}$ demonstrating the utility of such a technique in this kind of analysis. A novel semi quantitative magnetization transfer (MT) technique was developed recently ${ }^{71}$ with potentially greater sensitivity than standard MTR. The magnetization transfer saturation (MTsat) maps show an excellent contrast in the brain and with these highresolution MT maps can be overcome some of the MTR limitations like the influence of excitation and $T_{1}$ relaxation ${ }^{71,89}$. The main difference is that MTR refers to reduction of the steady-state signal, while MTsat refers to the saturation effect of a single MT pulse during the repetition time (TR) interval. Therefore, such MTsat maps are corrected for T1 relaxation changes. A wide range of MT values was observed in MS lesions, from liquid-like cores of acute and chronic lesions to subtle changes in the normal appearing white matter (NAWM). Subtle increased water content normally results in slower longitudinal relaxation and reduced MT. These effects amplify each other in the transient state but they partially cancel each other in the steady state. MTsat overcomes this cancelling effect and it can be a better estimate for those regions of interest without focal and acute lesions like the normal appearing spinal cervical cord tissue (NASC).

Gait impairment and balance are quantified using a combination of clinical examination and patients' reported maximum walking distance. The Kurtzke's functional systems and Expanded Disability Status Scale (EDSS) ${ }^{2}$ are widely used both in clinical practice and clinical trials. The EDSS provides a good overview of current neurologic status and mobility dysfunction. The timed 25 -foot walk (T25FW) measures the time a patient takes to walk 25 feet at maximum speed and it is currently 
the most widely implemented method to objectively quantify gait disability in clinical trials $^{3}$.

In this study we have explored the application of the MTsat maps to the cervical spinal cord of a cohort of patients with a diagnosis of MS searching to find their correlation with the level of disability reflected by EDSS score and T25FW. We compare results obtained using a standard MTR sequence.

\subsection{Methods}

\section{Patients}

This study was approved from the London - Chelsea Regional Ethics Committee and all patients gave their full informed consent. Any detail on the Patient Research Cohort Rapidly Evolving Multiple Sclerosis Study (PRC - REMS) was made publicly available in this site: http://clinicaltrials.gov/. Fifty-two patients were selected. The patients were selected among all those ones who had all the requirements needed to make the cervical cord MT maps and after discarding the cases affected by artifacts. Their ages were between 28-59 years old with a diagnosis of MS according to the revised McDonald's criteria ${ }^{91}$ with relapsing-remitting MS (RRMS) or secondary progressive MS (SPMS), and EDSS score (2.0-6.0) at screening (Table 6.1).

\section{MRI acquisition}

A 3T whole body MR system (Magnetom Verio, Siemens Medical Solutions, Erlangen, Germany) was used for the acquisition of the volumes. It was equipped with 12 channel head and 4 channel phased array neck coil. Several sequences were performed in order to get the volumes needed for the study as follows: T1-weighted 3D MPRAGE volumes before and after the Gadolinium contrast. They were acquired 
based on the ADNI-GO recommended parameters: $1 \mathrm{~mm}^{3}$ isotropic resolution, parallel imaging (PI) factor of 2 and 256x192 mm field of view (FOV), in a total time of 5:21 minutes. A physician injected of Gadolinium (Gadoterate meglumine, Dotarem, Guerbet, $0.1 \mathrm{mmol} / \mathrm{kg}$ ) 5 minutes before the acquisition of the post contrast volume. T2-weighted fluid attenuated inversion recovery 3D (FLAIR) volumes were acquired through the cord with $1 \mathrm{~mm}^{3}$ isotropic and 160 sagittal sections in a single

3D slab were acquired: 250x250 mm FOV, PI factor of 2, inversion time (TI) 1800 ms, repetition time (TR) 5s, and echo time (TE) $395 \mathrm{~ms}$.

MT maps for the CSC used a 3D FLASH sequence with $1 \mathrm{~mm}$ of isotropic resolution, 192 sagittal partitions, $256 * 240 \mathrm{FOV}$ and readout in the head-feet direction with $600 \mathrm{~Hz} /$ pixel bandwidth to reduce susceptibility artifacts. Six bipolar gradient echoes equally spaced between TE of 1.95 and $11.7 \mathrm{~ms}$ were acquired during a TR of $27 \mathrm{~ms}$. The iPAT factor of 2 was used in the GRAPPA parallel acquisition. A flip angle of $5^{\circ}$ was chosen for one of the PD-weighted dataset meanwhile another PD-w was MT-weighting. The magnetization was saturated by a band selective RF pulse of Gaussian shape with a duration of $12.24 \mathrm{~ms}, 2.2 \mathrm{kHz}$ frequency offset and nominal flip angle $540^{\circ}$.

We also acquired a T1-w volume with a TR of $15 \mathrm{~ms}$ and a flip angle of $18^{\circ}$. All volumes were spatially coregistered to the MPRAGE dataset to correct any movement between the acquisitions.

\section{MTsat maps}

In order to get the MTsat maps, two parameters need to be calculated. The first is the apparent proton density: 


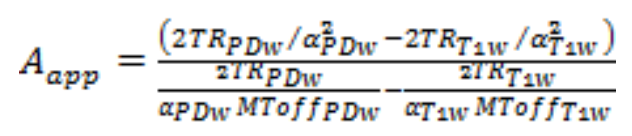

The second is the apparent $\mathrm{T} 1$ relaxation:

$$
T_{1 a p p}=\frac{\left(M T \circ f_{P D W} / \alpha_{P D w}-M T o f f_{T 1 w} / \alpha_{T 1 w}\right)}{M T o f f_{P D w} \alpha_{P D w} / 2 T R_{P D w}}-M T o f f_{T 1 w} \alpha_{T 1 w} / 2 T R_{T 1 w}
$$

The following equation gives the MTsat map:

$$
M T s a t=100 A_{a p p} \alpha_{M T w} \frac{T R_{M T w}}{T_{1 a p p}} / \text { MTon }_{p D w}-\frac{T R_{M T w}}{T_{1 a p p}}-\frac{\alpha_{M T w}^{2}}{2}
$$

The MTsat is an empirical equation for MT and it is the rational approximation of the Ernst equation under low values for the TR and the flip angles.

\section{MTR maps}

MTR is the percentage of reduction in the signal when the saturation is applied. Therefore, it is a relative measure:

$$
M T R=100\left(\text { MTof }_{P D w}-\text { MTon }_{P D w}\right) / M T o f f_{P D w}
$$

Where intensity of the PD-weighted without saturation is $M T o f f_{P D W}$ and the second with saturation is MTon $_{\text {PDw }}$.

\section{Data Analysis}

The CSC (C1/7) was extracted manually with Analyze 11.0 (AnalyzeDirect/Mayo

Clinic, KS). Linear registration was performed using the same software. The intensity distribution for the segmented MTsat and MTR maps were calculated by Matlab (Mathworks Natick, MA) and histograms were made for each subject. They were divided into bins of width 0.25 percent units (p.u.). Smoothing by convolution with a 
Gaussian kernel was performed over the histograms. Cervical lesions were marked manually with the help of the post Gadolinium volumes which were MPRAGE registered in order to make cervical focal enhancing lesion masks and they were identified in nineteen subjects.

Volumes were normalized with intracranial volume (ICV) to reduce interindividual variation and reduce subtle interimage differences. Inter-subjects coefficients of variation $(\mathrm{CoV})$ were calculated. Spearman rank-order correlation coefficient and Spearman's partial correlations (controlling for age, gender and disease duration) assessed associations between volume, MTR, MTsat, and both EDSS and T25FW. A two-tailed p value less than 0.05 was considered significant. Corrected thresholds for $\mathrm{p}$ were applied for multiple comparisons. All statistical analyses were performed with SPSS for Windows (v. 17, SPSS Inc., Chicago, Illinois).

\subsection{Results}

The study included 52 patients with multiple sclerosis (49 RRMS and 3 SPMS). Their median (range) for the age was 38 (59-28) years, disease duration was 2 (13-0) years, EDSS score was 3.5 (6-2), T25FW was 6.1 (14.1-4.1) seconds and they had a mean of 2 (4-0) relapses within the year (Table 6.1).

Cervical spinal cord volumes were normalized by ICV (SCF) and ranged between 0.011 and 0.006 . The mean cervical cord volume represented less than $1 \%$ of the ICV and the mean lesion volume was $0.124 \mathrm{~mL}$ (range: $0.266 \mathrm{~mL}-0.017 \mathrm{~mL}$ ) which was less than $0.01 \%$ of the mean ICV.

MTR and MTsat maps are shown in Figure 6.3. In the axial cut, a greater contrast of the central cord on the MTsat than the MTR can be appreciated. 
The whole and NASC MTR and MTsat histograms did not show different modes for CSF, WM and GM. (Figure 6.1 and Figure 6.2). Therefore, among the histogram features that are appropriate for analysis are peak height, peak location, mean and median parameter values ${ }^{90}$.

Small intra-subject variation was found in the parameter values for the mean/median cord and for segmented components for both MTR and MTsat (Table 6.2). Significant Spearman's correlations with EDSS were found for mean whole SC MTsat $(r=-0.293 ; p=0.035)$, median whole SC MTsat $(r=-0.278 ; p=0.046)$ (Table 6.3). No significant correlations were found for mean whole MTR $(r=-0.235 ; \mathrm{p}=$ 0.094) and median whole MTR ( $\mathrm{r}=-0.228 ; \mathrm{p}=0.104)$. Neither significant Spearman's partial correlations with T25FW for MTR nor MTsat were found. Spearman correlation for age, gender and disease duration adjusted and other results were summarized in the Table 6.4 and 6.6.

\subsection{Discussion}

These results show that pathology associated with different levels of disability can be discriminated based on MTsat of the CSC. The cervical cord is a crossroad for cerebrospinal descending and spinocerebral ascending pathways; damage to the cervical cord thus reflects widespread CNS functions. Conventional MR imaging is not fully sensitive to the heterogeneity of underlying histopathology, particularly in depicting pathologic changes within the NASC region which may include both axonal loss and demyelination. This may be the cause of the so-called clinico-radiological paradox in MS, by which conventional MRI measures do not fully explain disability distributions in populations ${ }^{110}$. 
MTR is a well-recognized MRI technique for studying MS because it provides quantitative microstructural information ${ }^{55,111}$. In general, MS pathology is associated with a decrease in MTR: low MTR has been found in lesions that were apparent on conventional MRI. Myelin loss (and, to a smaller extent, oedema) reduces MTR values in $\mathrm{MS}^{78}$. Several studies have shown that MTR histogram derived measures of the cervical cord correlate well with the clinical manifestations of $\mathrm{MS}^{112}$ and are related to demyelination ${ }^{113}$. This study shows significant correlations for MTsat metrics with EDSS. According to these results MTsat technique is sensitive to the presence of diffuse and mild changes of the NASC suggesting that progressive axonal damage or loss and/or demyelination could be responsible for functional impairment. Results of other studies ${ }^{114}$ indicate that axonal density variance is independent of differences in myelin density. With pathologically lower axon density, there is an increase in T1 relaxation time and a decrease in MTR. But within NASC, changes in relaxation times and MTR in lesions were dominated by myelin changes and less likely to independently reflect smaller contributions from axonal density variation.

The cervical cord volume also reflects remote volume by its reciprocal connectivity with distant brain and thoracolumbar cord axons and neurons. We found a lack of correlation of cord volume measures, MTR and MTsat metrics with the clinical score of ambulatory function $\mathrm{T} 25 \mathrm{FW}$. An explanation for this lack of correlation could be the restricted range in our sample and its mild ambulatory dysfunction (Figure 6.1). 


\subsection{Conclusion}

MTsat can discriminate between MS pathology associated with different disability levels in the cervical spinal cord in both whole cervical cord and normal appearing spinal cord. In this population, it appeared to be more sensitive to the variance than was MTR, which did not show correlations in the same regions. MTsat may provide a parameter more sensitive to the degree of tissue damage than is MTR. However, we recognise limitations to this study. The population size is relatively small and reflects a more aggressive disease population: the extent to which conclusions can be generalised is uncertain.

Table 6.1: Summary of clinical characteristics of the patients involved in the study. Medians with range.

\begin{tabular}{|c|c|}
\hline Subjects & 52 \\
\hline Age (years) & $38(59-28)$ \\
\hline Gender (f/m) & $41 / 11$ \\
\hline Disease Duration (years) & $2(13-0)$ \\
\hline EDSS & $3.5(6-2)$ \\
\hline T25FW (seconds) & $6.1(14.1-4.1)$ \\
\hline RRMS & 49 \\
\hline SPMS & 3 \\
\hline Relapses within last year & $2(4-0)$ \\
\hline SCF & $0.0088(0.0009)$ \\
\hline SCL (mL) & $0.124(0.089)$ \\
\hline
\end{tabular}

Median (range) values are shown for: age, disease duration, EDSS, T25FW and relapses within last year. Mean (standard deviation) values are for: SCF and SCL. Abbreviations: EDSS = Expanded Disability Status Scale, T25FW = timed 25-foot walk, RRMS = relapsing remitting multiple sclerosis, $\mathrm{SPMS}=$ secondary progressive multiple sclerosis, $\mathrm{SCF}=$ spinal cord fraction, $\mathrm{SCL}=$ spinal cord lesions. 
Table 6.2: MTR and MTsat histogram metrics. All in p.u (Peak height is normalized by the total number of voxels)

\begin{tabular}{|c|c|c|c|c|}
\hline & MTR & CoV & MTsat & CoV \\
\hline & & & & \\
\hline Mean whole SC & $39.24(3.30)$ & 8.41 & $16.62(2.34)$ & 14.08 \\
\hline Mean NASC & $39.98(3.70)$ & 9.24 & $16.74(2.32)$ & 13.89 \\
\hline & & & & \\
\hline Median whole SC & $41.44(3.38)$ & 8.16 & $17.25(2.78)$ & 16.12 \\
\hline Median NASC & $42.10(3.79)$ & 9.01 & $17.48(2.74)$ & 15.67 \\
\hline Peak location whole SC & $47.16(3.72)$ & 7.89 & $19.09(3.07)$ & 16.61 \\
\hline & & & & \\
\hline Peak height whole SC & $0.023(0.005)$ & 21.74 & $0.029(0.005)$ & 17.24 \\
\hline
\end{tabular}

Abbreviations: MTR $=$ magnetization transfer ratio, MTsat $=$ magnetization transfer saturation, $\mathrm{CoV}=$ coefficient of variation, $\mathrm{SC}=$ cervical spinal cord, $\mathrm{NASC}=$ normal appearing cervical spinal cord. 
Table 6.3: Spearman rank-order correlation with EDSS for MTR and MTsat ${ }^{*} \mathrm{p}<$ $0.05)$

\begin{tabular}{|c|c|c|}
\hline & \multicolumn{2}{|c|}{ EDSS } \\
\hline MTR & $\mathrm{r}$ & p Value \\
\hline Mean whole SC & -0.235 & 0.094 \\
\hline Mean NASC & -0.271 & 0.052 \\
\hline Mean SCL & -0.386 & 0.102 \\
\hline Median whole SC & -0.228 & 0.104 \\
\hline Median NASC & -0.241 & 0.085 \\
\hline Median SCL & -0.380 & 0.109 \\
\hline Peak location whole SC & -0.251 & 0.073 \\
\hline Peak location SCL & -0.376 & 0.113 \\
\hline Peak height whole SC & -0.465 & $0.001 *$ \\
\hline Peak height SCL & -0.199 & 0.415 \\
\hline MTsat & $\mathrm{r}$ & $\mathrm{p}$ Value \\
\hline Mean whole SC & -0.293 & $0.035^{*}$ \\
\hline Mean NASC & -0.354 & $0.010 *$ \\
\hline Mean SCL & -0.305 & 0.205 \\
\hline Median whole SC & -0.278 & $0.046^{*}$ \\
\hline Median NASC & -0.333 & $0.016^{*}$ \\
\hline Median SCL & -0.468 & $0.043^{*}$ \\
\hline Peak location whole SC & -0.187 & 0.185 \\
\hline Peak location SCL & -0.410 & 0.081 \\
\hline Peak height whole SC & -0.377 & $0.006^{*}$ \\
\hline Peak height SCL & -0.014 & 0.954 \\
\hline
\end{tabular}

Abbreviations: EDSS $=$ Expanded Disability Status Scale, MTR = magnetization transfer ratio, MTsat = magnetization transfer saturation, $\mathrm{SC}=$ cervical spinal cord, $\mathrm{NASC}=$ normal appearing cervical spinal cord, $\mathrm{SCL}=$ cervical spinal cord lesions . 
Table 6.4: Spearman's partial correlations (control variables)) with EDSS for MTR and MTsat $(* * \mathrm{p}<0.017$ and $* * * \mathrm{p}<0.005 ;$ Bonferroni corrected $\mathrm{p}$ value thresholds).

\begin{tabular}{|c|c|c|c|c|}
\hline & \multicolumn{4}{|c|}{ EDSS } \\
\hline & \multicolumn{2}{|c|}{$\begin{array}{l}\text { Partial correlations } \\
\text { (age) }\end{array}$} & \multicolumn{2}{|c|}{$\begin{array}{l}\text { Partial correlations } \\
\text { (age, gender, disease } \\
\text { duration) }\end{array}$} \\
\hline MTR & $\mathrm{r}$ & $\mathrm{p}$ Value & $\mathrm{r}$ & $\mathrm{p}$ Value \\
\hline Mean whole SC & -0.242 & 0.087 & -0.346 & 0.016 \\
\hline Mean NASC & -0.277 & 0.049 & -0.409 & $0.004 * * *$ \\
\hline Mean SCL & -0.270 & 0.279 & -0.512 & 0.043 \\
\hline Median whole SC & -0.230 & 0.104 & -0.335 & 0.020 \\
\hline Median NASC & -0.257 & 0.068 & -0.378 & 0.008 \\
\hline Median SCL & -0.293 & 0.238 & -0.486 & 0.056 \\
\hline Peak location whole SC & -0.290 & 0.039 & -0.401 & 0.005 \\
\hline Peak location SCL & -0.331 & 0.180 & -0.500 & 0.048 \\
\hline Peak height whole SC & -0.442 & $0.001 * *$ & -0.601 & $<0.001 * * *$ \\
\hline Peak height SCL & -0.074 & 0.769 & -0.054 & 0.841 \\
\hline MTsat & $\mathrm{r}$ & $\mathrm{p}$ Value & $\mathrm{r}$ & $\mathrm{p}$ Value \\
\hline Mean whole SC & -0.341 & $0.014 * *$ & -0.394 & 0.006 \\
\hline Mean NASC & -0.321 & $0.005 * *$ & -0.437 & $0.002 * * *$ \\
\hline Mean SCL & -0.213 & 0.397 & -0.423 & 0.103 \\
\hline Median whole SC & -0.328 & 0.019 & -0.371 & 0.009 \\
\hline Median NASC & -0.368 & $0.008 * *$ & -0.422 & $0.003 * * *$ \\
\hline Median SCL & -0.397 & 0.103 & -0.539 & 0.031 \\
\hline Peak location whole SC & -0.234 & 0.099 & -0.277 & 0.057 \\
\hline Peak location SCL & -0.354 & 0.149 & -0.494 & 0.052 \\
\hline Peak height whole SC & -0.442 & $0.001 * *$ & -0.489 & $<0.001 * * *$ \\
\hline Peak height SCL & 0.087 & 0.733 & -0.279 & 0.296 \\
\hline
\end{tabular}

Abbreviations: EDSS $=$ Expanded Disability Status Scale, MTR $=$ magnetization transfer ratio, MTsat = magnetization transfer saturation, $\mathrm{SC}=$ cervical spinal cord, $\mathrm{NASC}=$ normal appearing cervical spinal cord, $\mathrm{SCL}=$ cervical spinal cord lesions. 
Table 6.5: Spearman rank-order correlation coefficients with T25FW for MTR and MTsat $(* p<0.05)$.

\begin{tabular}{|c|c|c|}
\hline & \multicolumn{2}{|c|}{$\mathrm{T} 25 \mathrm{FW}$} \\
\hline MTR & $\mathrm{r}$ & $\mathrm{p}$ Value \\
\hline Mean whole SC & -0.039 & 0.785 \\
\hline Mean NASC & -0.038 & 0.792 \\
\hline Mean SCL & -0.261 & 0.280 \\
\hline Median whole SC & -0.055 & 0.700 \\
\hline Median NASC & -0.018 & 0.900 \\
\hline Median SCL & -0.275 & 0.254 \\
\hline Peak location whole SC & -0.065 & 0.645 \\
\hline Peak location SCL & -0.229 & 0.346 \\
\hline Peak height whole SC & -0.190 & 0.176 \\
\hline Peak height SCL & -0.281 & 0.243 \\
\hline MTsat & $\mathrm{r}$ & $\mathrm{p}$ Value \\
\hline Mean whole SC & -0.087 & 0.541 \\
\hline Mean NASC & -0.124 & 0.381 \\
\hline Mean SCL & -0.319 & 0.183 \\
\hline Median whole SC & -0.094 & 0.506 \\
\hline Median NASC & -0.128 & 0.365 \\
\hline Median SCL & -0.378 & 0.110 \\
\hline Peak location whole SC & -0.153 & 0.280 \\
\hline Peak location SCL & -0.337 & 0.158 \\
\hline Peak height whole SC & -0.090 & 0.525 \\
\hline Peak height SCL & -0.037 & 0.881 \\
\hline
\end{tabular}

Abbreviations: $\mathrm{T} 25 \mathrm{FW}=$ timed 25 -foot walk, $\mathrm{MTR}=$ magnetization transfer ratio, MTsat $=$ magnetization transfer saturation, $\mathrm{SC}=$ cervical spinal cord, $\mathrm{NASC}=$ normal appearing cervical spinal cord, $\mathrm{SCL}=$ cervical spinal cord lesions. 
Table 6.6: Spearman's partial correlations (control variables) with T25FW for MTR and MTsat $(* * \mathrm{p}<0.017$ and $* * * \mathrm{p}<0.005$; Bonferroni corrected $\mathrm{p}$ value thresholds)

\begin{tabular}{|c|c|c|c|c|}
\hline & \multicolumn{4}{|c|}{$\mathrm{T} 25 \mathrm{FW}$} \\
\hline & \multicolumn{2}{|c|}{$\begin{array}{l}\text { Partial correlations } \\
\text { (age) }\end{array}$} & \multicolumn{2}{|c|}{$\begin{array}{l}\text { Partial correlations } \\
\text { (age, gender, } \\
\text { disease duration) }\end{array}$} \\
\hline MTR & $\mathrm{r}$ & p Value & $\mathrm{r}$ & $\mathrm{p}$ Value \\
\hline Mean whole SC & -0.035 & 0.808 & -0.099 & 0.503 \\
\hline Mean NASC & -0.030 & 0.832 & -0.108 & 0.463 \\
\hline Mean SCL & -0.059 & 0.816 & -0.175 & 0.516 \\
\hline Median whole SC & -0.047 & 0.743 & -0.115 & 0.438 \\
\hline Median NASC & -0.021 & 0.881 & -0.096 & 0.516 \\
\hline Median SCL & -0.132 & 0.545 & -0.192 & 0.476 \\
\hline Peak location whole SC & -0.093 & 0.517 & -0.158 & 0.282 \\
\hline Peak location SCL & -0.153 & 0.545 & -0.189 & 0.483 \\
\hline Peak height whole SC & -0.149 & 0.296 & -0.253 & 0.083 \\
\hline Peak height SCL & -0.122 & 0.629 & -0.146 & 0.590 \\
\hline MTsat & $\mathrm{r}$ & $\mathrm{p}$ Value & $\mathrm{r}$ & $\mathrm{p}$ Value \\
\hline Mean whole SC & -0.121 & 0.398 & -0.145 & 0.325 \\
\hline Mean NASC & -0.143 & 0.318 & -0.169 & 0.252 \\
\hline Mean SCL & -0.182 & 0.470 & -0.273 & 0.306 \\
\hline Median whole SC & -0.132 & 0.356 & -0.151 & 0.305 \\
\hline Median NASC & -0.151 & 0.289 & -0.178 & 0.226 \\
\hline Median SCL & -0.265 & 0.288 & -0.305 & 0.250 \\
\hline Peak location whole SC & -0.197 & 0.167 & -0.225 & 0.124 \\
\hline Peak location SCL & -0.254 & 0.308 & -0.292 & 0.272 \\
\hline Peak height whole SC & -0.136 & 0.341 & -0.163 & 0.268 \\
\hline Peak height SCL & 0.061 & 0.810 & 0.119 & 0.662 \\
\hline
\end{tabular}

Abbreviations: $\mathrm{T} 25 \mathrm{FW}=$ timed 25-foot walk, $\mathrm{MTR}=$ magnetization transfer ratio, MTsat = magnetization transfer saturation, $\mathrm{SC}=$ cervical spinal cord, $\mathrm{NASC}=$ normal appearing cervical spinal cord, SCL = cervical spinal cord lesions. 


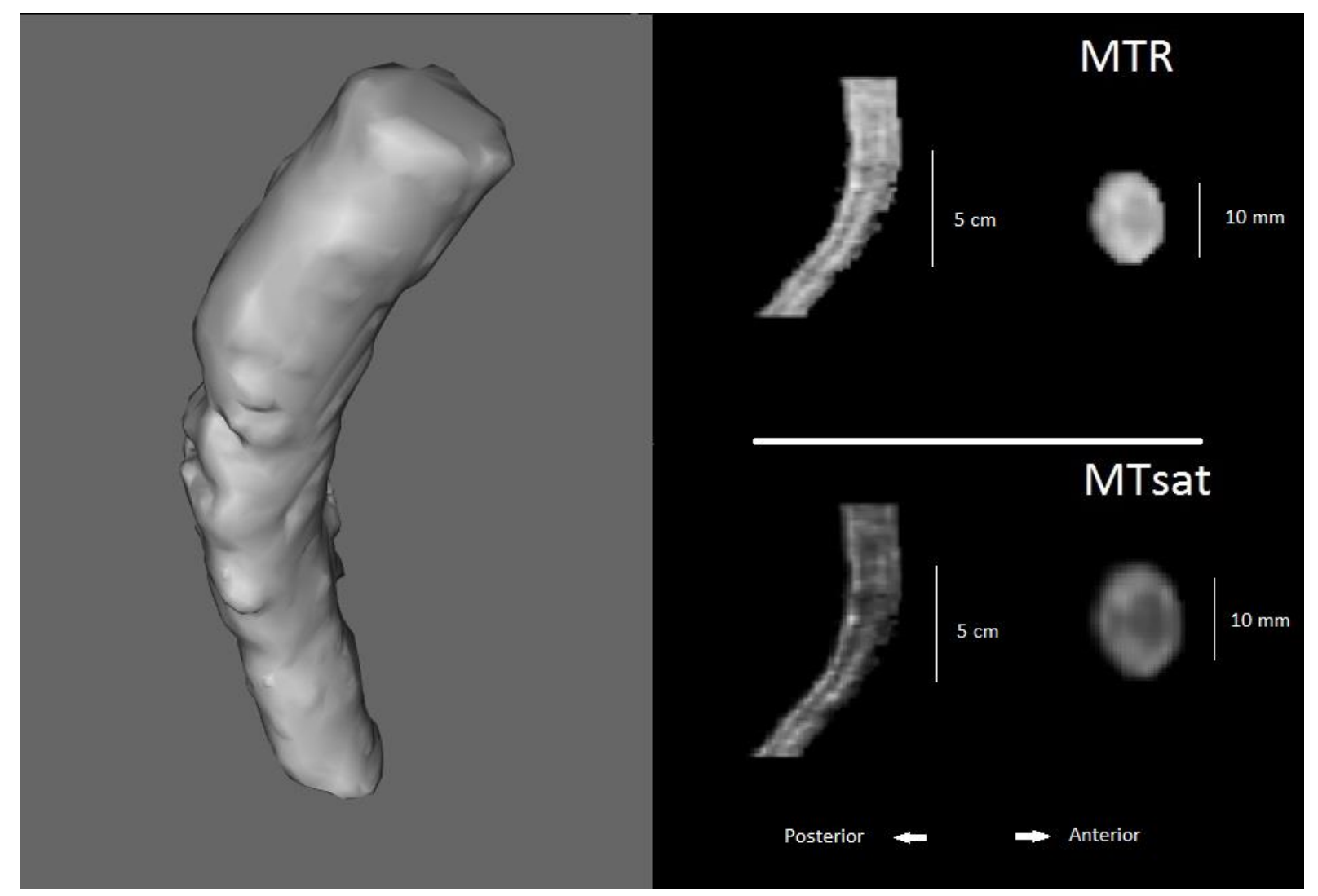

Figure 6.1: On the left, 3D representation of the volume of the cervical cord. On the right, MTR and MTsat maps with axial detail of C3. MTsat can improve the contrast in the inner structures and shows correlation with EDSS. Spinal cord atrophy measurements still are not commonly included in MRI protocols, most likely due to practical reasons: inclusion of both brain and cord imaging in the same MRI examination greatly prolongs the scanning time and the challenging of the cord segmentation methods.

Abbreviations: MTR = magnetization transfer ratio, MTsat $=$ magnetization transfer saturation, EDSS = Expanded Disability Status Scale. 
Figure 6.2: Average whole cervical cord MTR and MTsat histograms.

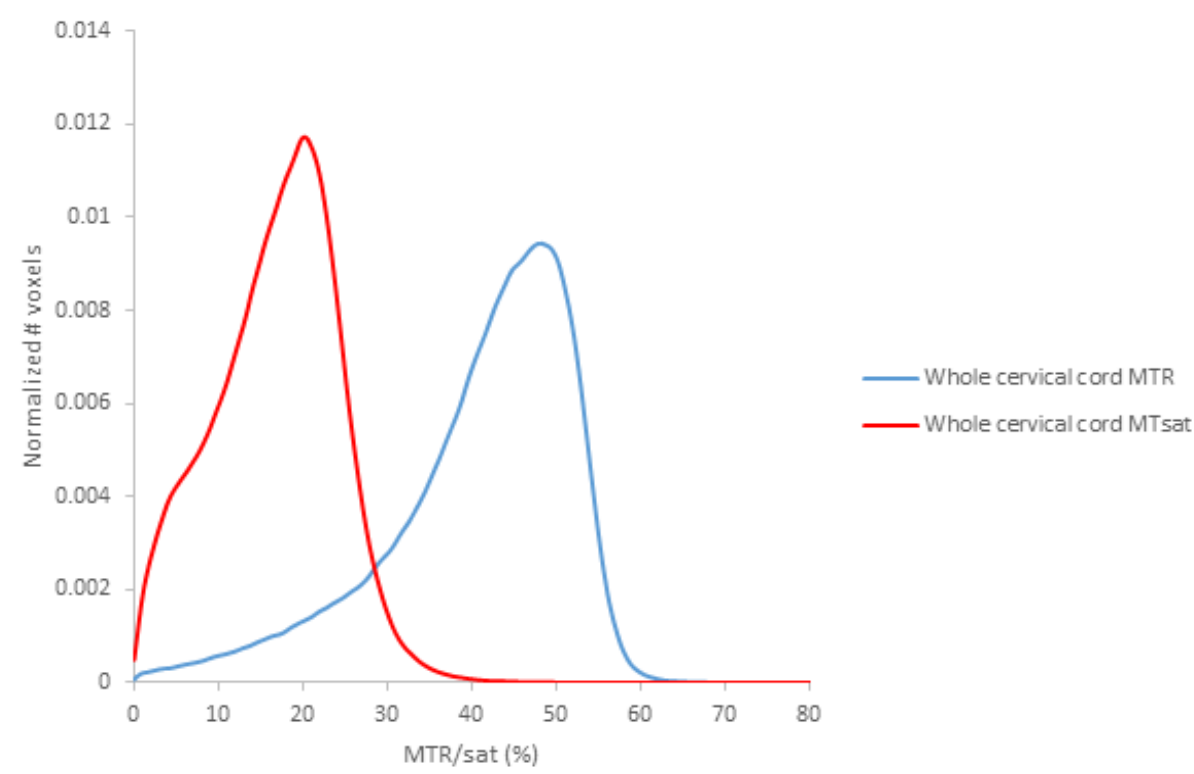

Abbreviations: MTR $=$ magnetization transfer ratio, MTsat $=$ magnetization transfer saturation.

Figure 6.3: Average NASC and SCL MTR/sat histograms

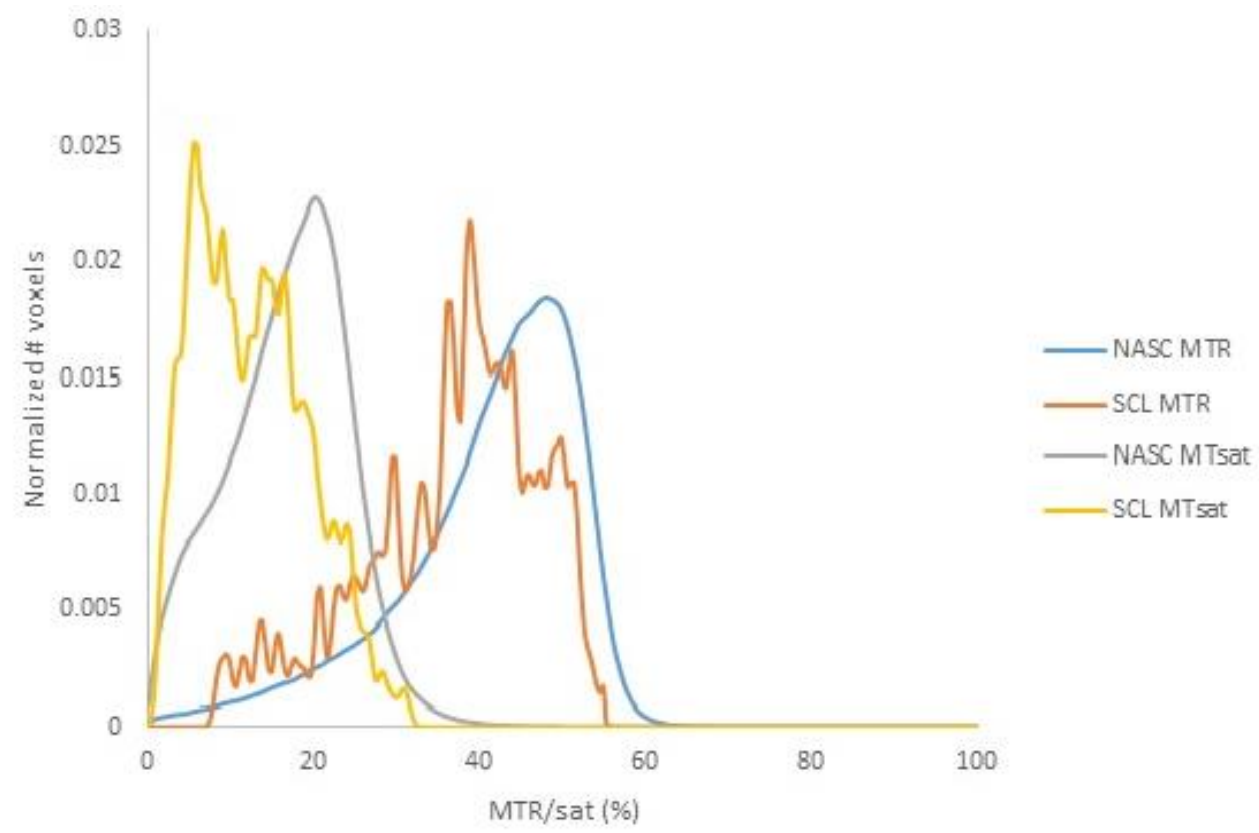

Abbreviations: MTR $=$ magnetization transfer ratio, MTsat $=$ magnetization transfer saturation, NASC = normal appearing spinal cord, SCL = spinal cord lesions. 
Figure 6.4: Scatter plots of whole MTsat against EDSS.

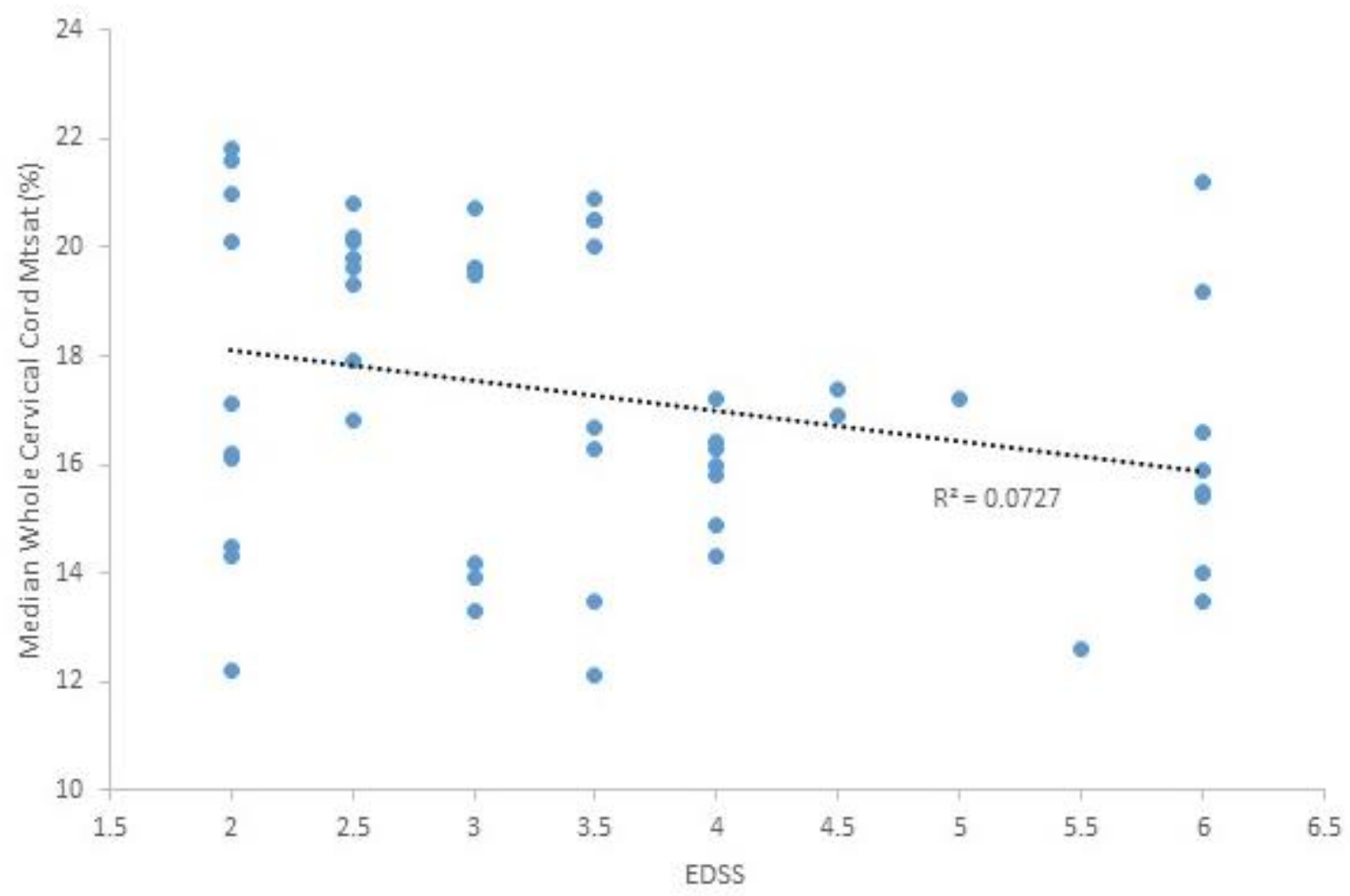

Abbreviations: MTsat $=$ magnetization transfer saturation, EDSS $=$ Expanded Disability Status Scale. 


\section{Chapter 7}

\section{Relationships between microstructure of the}

\section{brain and cervical cord across different levels of}

\section{disability in patients with multiple sclerosis:}

\section{atrophy and MT histogram analyses}

\subsection{Introduction}

Multiple sclerosis (MS) produces both inflammatory and degenerative pathology of brain and spinal cord. Histological studies showed there is neuronal loss in the cortical and deep grey matter (GM), axonal loss in white matter (WM) and also in the cervical spinal cord ${ }^{27,115-118}$. Conventional magnetic resonance imaging of the brain is highly sensitive for detecting focal lesions in patients with $\mathrm{MS}^{119}$. Therefore, the assessment of the burden of brain $\mathrm{T} 2$ lesions is used to monitor evolution of $\mathrm{MS}^{74}$. However the magnitude of the correlation between brain MRI lesion load and clinical disability is only modest ${ }^{99}$. Spinal cord MRI is less sensitive than brain MRI for detecting MS lesions ${ }^{113,120}$, but there is evidence that the occurrence of MS lesions in the cord gives rise to clinical symptoms more often than lesions located in the brain ${ }^{121}$. The assessment of MS pathology using T2 weighted images is limited by the poor specificity of this technique to the heterogeneous substrates of MS lesions ${ }^{122}$ and by 
its inability to provide information about the microscopic changes occurring in the normal appearing white matter (NAWM) ${ }^{95}$.

Magnetisation transfer imaging (MTI) provides quantitative metrics which are sensitive to the most destructive aspects of MS pathology $y^{69,123}$ and allows macroscopic and microscopic disease burdens to be estimated ${ }^{35}$. MTI reflects the exchange of magnetization between freely mobile protons and those associated with macromolecules in myelin or axonal membranes ${ }^{124}$. For example, low MT ratio (MTR) is strongly correlated with the percentage of residual myelin and axons present in MS lesions and $\mathrm{NAWM}^{55}$. In addition to the standard MTR parameter for magnetization, the novel quantitative MT saturation (MTsat) parameter is of particular interest as, unlike MTR, it is minimally affected by T1 relaxation, is less sensitive to B1 inhomogeneity, and WM/GM contrast in MTsat maps is increased relative to that in MTR maps ${ }^{71}$. Therefore, the MTsat method has the potential to provide insight into pathological processes occurring in the cervical cord affected by neurological disorder such as MS.

The relationship between the destructive processes in MS that affect the brain versus the spinal cord in the same subjects is not well defined. The goals of this study are to evaluate the connection between brain and cervical cord atrophy with disability, to relate this to correlations between MTR and MTsat histogram metrics of the brain and the cervical cord and to investigate the relationship between brain pathology and cervical cord MTR/MTsat histogram metrics with disability. 


\subsection{Methods}

\section{Patients}

The cohort of patients was taken from the Patient Research Cohort Rapidly Evolving Multiple Sclerosis Study (PRC - REMS), which has the approval of the London Chelsea Regional Ethics Committee. For this study, forty-three MS patients were selected within two phenotypes: relapsing-remitting MS (RRMS) and secondary progressive MS (SPMS). The patients were selected among all those whose examinations provided adequate brain and cervical cord MT maps after discarding the cases affected by artifacts. The degree of disability was estimated according to the Kurtzke's Expanded Disability Status Scale (EDSS) and the Timed 25-Foot Walk (T25FW) scale from the Multiple Sclerosis Functional Composite (MSFC).

\section{MRI acquisition}

The study was performed on a 3T clinical MR system (Magneton Verio, Siemens Medical Solutions, Erlangen, Germany). A twelve channel phased array head coil and four channel phased array neck coil were used for reception. T1-weighted 3D MPRAGE volumes were acquired with these parameters: $1 \mathrm{~mm}^{3}$ isotropic resolution, parallel imaging (PI) factor of 2 and 256x192 mm field of view (FOV). A physician injected of Gadolinium (Gadoterate meglumine, Dotarem, Guerbet, $0.1 \mathrm{mmol} / \mathrm{kg}$ ) 5 minutes before the acquisition of the post contrast volume. T2-weighted Fluid Attenuated Inversion Recovery (FLAIR) volumes were acquired with 1 $\mathrm{mm}^{3}$ isotropic and 160 sagittal sections in a single 3D slab, 250x250 mm FOV, PI

factor of 2, inversion time (TI) $1800 \mathrm{~ms}$, repetition time (TR) 5s, and echo time (TE) $395 \mathrm{~ms}$. 3D FLASH sequence with $1 \mathrm{~mm}$ of isotropic resolution, 192 sagittal 
partitions, 256*240 FOV and readout in the head-feet direction with $600 \mathrm{~Hz} /$ pixel bandwidth to reduce susceptibility artifacts. Six bipolar gradient echoes equally spaced between TE of 1.95 and $11.7 \mathrm{~ms}$ were acquired during a TR of $27 \mathrm{~ms}$. The iPAT factor of 2 was used in the GRAPPA parallel acquisition. A flip angle of $5^{\circ}$ was chosen for one of the PD-weighted dataset meanwhile another PD-w was MTweighting. The magnetization was saturated by a band selective RF pulse of Gaussian shape with a duration of $12.24 \mathrm{~ms}, 2.2 \mathrm{kHz}$ frequency offset and nominal flip angle $540^{\circ}$. T1-w volume with a TR of $15 \mathrm{~ms}$ and a flip angle of $18^{\circ}$.

\section{Data Analysis}

MTR maps were produced on a voxel by voxel basis according to the equation:

$$
M T R=100 \frac{M_{0}-M_{S}}{M_{0}}
$$

Where $M_{s}$ and $M_{0}$ are signal intensities with application of saturation pulse and without respectively. MTR values are reported in percent units (p.u). The MTsat parameter was calculated by inserting the estimated $A_{a p p}$ and $T_{1 a p p}$ values in the approximate signal equation for the MT FLASH experiment ${ }^{71}$. The MTsat parameter represents the additional percentage reduction in the steady state FLASH signal caused by the saturation effect of a single MT pulse. It is calculated from this equation:

$$
\operatorname{MTon}_{p D w}=A_{a p p} \alpha\left(T R / T_{1 a p p}\right) /\left(T R / T_{1 a p p}+\alpha^{2} / 2+M T s a t\right)
$$

Using $A_{a p p}$ and $T_{1 a p p}$ obtained from this equation: 


$$
\operatorname{MTon}_{p D w}=A_{\text {app }} \alpha\left(T R / T_{1 a p p}\right) /\left(T R / T_{1 a p p}+\alpha^{2} / 2\right)
$$

MTsat has been shown to be insensitive to inhomogeneities in RF transmit field and receive fields. Also, unlike MTR, the MTsat is not affected by T1 relaxation.

An automated technique based on prior probabilities was used on T1-w images in the extraction of the brain and segmentation of GM and WM. The cervical cord was extracted manually with Analyze 11.0 (AnalyzeDirect/Mayo Clinic, KS). Linear registration was performed using the same software. The intensity distribution for the segmented MTsat and MTR maps were calculated by Matlab (Mathworks Natick, MA) and histograms were made for each subject. They were divided into bins of width 0.25 percent units (p.u.). Smoothing by convolution with a Gaussian kernel was performed over the histograms. Linear registration of datasets, brain extraction and arithmetic calculations were performed using (Flirt, Sienax, FSL, Centre for Functional Magnetic Resonance Imaging of the Brain, University of Oxford, UK). WM lesions were marked manually and extracted with Analize. T2 hyperintensities of the FLAIR volumes were marked and co-registered in order to make the masks to get the NAWM. They were identified in forty-three cases. Cervical lesions were marked manually with the help of the post Gadolinium volumes which were MPRAGE registered in order to make cervical focal enhancing lesion masks and they were identified in sixteen subjects.

Volumes were normalized with intracranial volume (ICV) to reduce interindividual variation and reduce subtle interimage differences. Spearman rankorder correlation coefficient and Spearman's partial correlations (controlling for age, gender and disease duration) assessed associations between volumes, MTR, MTsat, and both EDSS and T25FW. 
Multivariate regressions were used to evaluate the effect of cervical cord MTR/MTsat histogram measures and T2 lesion volume (T2LV) on the disability measured by EDSS and T25FW.

A two tailed $\mathrm{p}$ value less than 0.05 was considered significant and corrected thresholds for $\mathrm{p}$ were applied for multiple comparisons. All statistical analyses were performed with SPSS for Windows (v. 17, SPSS Inc., Chicago, Illinois).

\subsection{Results}

The study included 43 patients with MS (40 RRMS and 3 SPMS). Their median (range) in age was 38 (59-28) years, disease duration was 2 (13-0) years, EDSS score was 3 (6-2) and T25FW was 5.9 (14.1-4.1) seconds. They had a median of 2 (4-0) relapses within the year (Table 7.1).

Significant Spearman correlations were found for the total brain lesion volume (T2LV) with EDSS $(r=0.357 ; p=0.019)$ and cervical cord fractional volume with T25FW ( $\mathrm{r}=-0.339 ; \mathrm{p}=0.026)$, although neither explains substantial proportions of the total variance in disability (Table 7.2). Scatter plots of brain and cord volumes are shown in Figure 7.2. However, for individual patients, there was no correlation between brain T2LV and the cervical cord fraction volume (Figure 7.3). Images illustrating this are shown in Figure 7.1 for two patients.

The histogram metrics median, mean, peak height and peak location of the whole brain, NAWM, whole cervical cord and NASC MTR/MTsat histograms were analyzed. None of the correlations between MTR/MTsat histogram derived metrics obtained from the brain and the cervical cord were significant after correction. In two cases we found a trend toward significant correlations (i.e., those that reached $\mathrm{p}<$ 
0.017, the Bonferroni corrected $\mathrm{p}$ value threshold): Spearman's partial correlation (control variable: age) between mean whole brain MTsat and peak height whole cervical cord MTsat $(r=0.357 ; \mathrm{p}=0.020)$ and median whole brain MTsat and peak height whole cervical cord MTsat $(\mathrm{r}=0.348 ; \mathrm{p}=0.024)$.

We explored multivariate regressions (Tables 7.4 and 7.5), controlling for age, gender and brain T2LV, we found that the median whole cervical cord MTsat was significantly explained a proportion of the variation of EDSS as a dependent variable, but a similar relationship was not found for T25FW.

\subsection{Discussion}

Previous studies found T2 lesion volume in the brain or spinal cord have not tended to show strong or significant correlations with the $\operatorname{EDSS}^{125,126}$, although, in our population, there was a significant Spearman significant correlation of brain T2LV with EDSS. By contrast, we did not find simple correlation between brain and cord volumes with EDSS and T25FW, although we found a significant correlation of the SCF with T25FW. Failure to identify associations reported by others ${ }^{127}$ may reflect the restricted range of our sample of patients, who were mildly disabled (median EDSS $=3$ ), with a well preserved ambulatory function (median $\mathrm{T} 25 \mathrm{FW}=5.9$ seconds) and shorter disease duration (median disease duration $=2$ years); studies which involved larger cohorts with a longer disease duration ( $>20$ years) and higher EDSS (median of 4, range 0-8) have reported associations between upper spinal cord cross sectional area and disability ${ }^{127}$. Additional, technical considerations also may have influenced the outcome of our study. There are significant challenges to performing reliable measurement of spinal cord cross sectional area and to its segmentation. The cord is small in relation to the voxel resolution, so partial volume 
effects are significant and care must be taken to align the imaging planes exactly perpendicular to the cord and to select consistent landmarks (vertebral discs usually) to frame measurements.

Significant relationship between brain and cervical cord volumes were not found and this suggest a non-uniform disease process in MS. The inflammatory processes may affect each region independently or show differential involvement between patients. In the brain tissue, axonal transection has been documented at the center and the periphery of demyelinating lesions ${ }^{128}$ leading to axonal and tissue volume loss in the adjacent WM, but in a large cross sectional MRI study ${ }^{113}$ and in a post mortem study ${ }^{129}$ no relationship between spinal cord atrophy and brain lesions was found.

There is a complex dependency of volume measures on tissue destruction in the brain and cervical cord. Brain or cervical cord volume are determined by multiple factors: tissue loss, inflammatory infiltration, edema and toxic agents. In an earlier study of a broad population of MS patients ${ }^{130}$, a trend toward increased spinal cord volume throughout the cervical cord in RRMS reached statistical significance with a statistically significant decrease in spinal cord volume at the upper cervical levels in PPMS and in the group with SPMS vs RRMS. Confounds of inflammatory edema related cord expansion may be greater in RRMS patients.

Our results did not demonstrate a correlation between brain pathology measures and cervical cord MTR/MTsat histogram metrics, but a trend toward significant correlation were found in the cases of the median and mean whole brain MTsat and the peak height whole cervical cord MTsat. Pathological studies, which assessed mostly SPMS and PPMS patients, have demonstrated that individual cord lesions play a minor role in local atrophy ${ }^{116}$. This suggest that Wallerian degeneration 
of long fiber tracts, rather than local damage to the tracts, may be one of the main factors leading to tissue loss in the cord with MS. Also, previous studies ${ }^{113}$ found very modest correlations between the extent of brain lesions and the cord pathology, suggesting that Wallerian degeneration has a minor role in the cervical cord abnormalities in MS. Our results therefore suggest that MTsat could be sensitive to some of these second order effects reflecting the Wallerian degeneration of fiber tracts crossing the brain lesions, although this needs direct confirmation.

The non-significant correlation between brain cMRI and cervical cord MTR/MTsat measures indicates that MS abnormalities from these modalities are largely independent of each other.

\subsection{Conclusions}

In a cohort of mildly disabled MS patients, the weak relationship between cervical cord and brain volume measures suggests that atrophy in the brain and cord progress independently in patients with MS. Nevertheless, the trend toward significant correlation between some brain and cervical cord MTsat metrics, suggests some global relationships in burden of disease. 
Table 7.1: Summary of clinical characteristics of the patients involved in the study.

\begin{tabular}{|c|c|}
\hline Subjects & 43 \\
\hline Age (years) & $38(59-28)$ \\
\hline Gender (f/m) & $36 / 7$ \\
\hline Disease Duration (years) & $2(13-0)$ \\
\hline EDSS & $3(6-2)$ \\
\hline T25FW & $5.9(14.1-4.1)$ \\
\hline RRMS & 40 \\
\hline SPMS & 3 \\
\hline Relapses within last year & $2(4-0)$ \\
\hline WMF & $0.38(0.02)$ \\
\hline GMF & $0.37(0.04)$ \\
\hline BPF & $0.75(0.04)$ \\
\hline SCF & $0.0089(0.0009)$ \\
\hline T2LV (mL) & $17.9(11.9)$ \\
\hline SCL (mL) & $0.127(0.082)$ \\
\hline
\end{tabular}

Median (range) values are shown for: age, disease duration, EDSS, T25FW and relapses within last year. Mean (standard deviation) values are for: WMF, GMF, BPF, SCF, T2LV and SCL.

Abbreviations: EDSS = Expanded Disability Status Scale, T25FW = timed 25-foot walk, RRMS = relapsing remitting multiple sclerosis, SPMS = secondary progressive multiple sclerosis, $\mathrm{WMF}=$ white matter fraction, $\mathrm{GMF}=$ grey matter fraction, $\mathrm{BPF}=$ brain parenchyma fraction, $\mathrm{SCF}=$ cervical spinal cord fraction, $\mathrm{T} 2 \mathrm{LV}=\mathrm{T} 2$ lesion volume, SCL = cervical spinal cord lesions. 
Table 7.2: Spearman rank-order correlations for fractional volumes and total lesion volumes with EDSS and T25FW $(* \mathrm{p}<0.05)$

\begin{tabular}{|c|c|c|}
\hline & \multicolumn{2}{|c|}{ EDSS } \\
\hline & $\mathrm{r}$ & $\mathrm{p}$ Value \\
\hline WMF & 0.278 & 0.071 \\
\hline GMF & -0.101 & 0.520 \\
\hline BPF & -0.018 & 0.910 \\
\hline T2 LV & 0.357 & $0.019^{*}$ \\
\hline SCF & -0.062 & 0.692 \\
\hline SCL & -0.368 & 0.161 \\
\hline \multicolumn{2}{|c|}{ T25FW } \\
\hline & $\mathrm{r}$ & $\mathrm{p}$ Value \\
\hline WMF & 0.159 & 0.309 \\
\hline GMF & -0.250 & 0.106 \\
\hline BPF & -0.199 & 0.200 \\
\hline T2 LV & 0.173 & 0.268 \\
\hline SCF & -0.339 & $0.026^{*}$ \\
\hline SCL & -0.145 & 0.593 \\
\hline
\end{tabular}

Abbreviations: EDSS = Expanded Disability Status Scale, T25FW = timed 25-foot walk, $\mathrm{WMF}=$ white matter fraction, $\mathrm{GMF}=$ grey matter fraction, $\mathrm{BPF}=$ brain parenchyma fraction, $\mathrm{SCF}=$ cervical spinal cord fraction, $\mathrm{T} 2 \mathrm{LV}=\mathrm{T} 2$ lesion volume, $\mathrm{SCL}=$ cervical spinal cord lesions. 
Table 7.3: Spearman's partial correlations (control variables) with EDSS and T25FW $(* * \mathrm{p}<0.017$ and $* * * \mathrm{p}<0.005$; Bonferroni corrected $\mathrm{p}$ value thresholds).

\begin{tabular}{|c|c|c|c|c|}
\hline & \multicolumn{5}{|c|}{ EDSS } \\
\hline & \multicolumn{2}{|c|}{ Partial correlation (age) } & \multicolumn{2}{c|}{$\begin{array}{c}\text { Partial correlation (age, } \\
\text { gender, disease duration) }\end{array}$} \\
\hline & $\mathrm{r}$ & $\mathrm{p}$ Value & $\mathrm{r}$ & $\mathrm{p}$ Value \\
\hline WMF & 0.213 & 0.176 & 0.073 & 0.655 \\
\hline GMF & 0.019 & 0.904 & 0.089 & 0.584 \\
\hline BPF & 0.065 & 0.684 & 0.056 & 0.730 \\
\hline T2 LV & 0.328 & 0.034 & 0.247 & 0.125 \\
\hline SCF & -0.019 & 0.905 & 0.013 & 0.938 \\
\hline SCL & -0.443 & 0.098 & -0.394 & 0.131 \\
\hline \multicolumn{5}{|c|}{ T25FW } \\
\hline WMF & 0.082 & 0.606 & -0.034 & 0.837 \\
\hline GMF & -0.150 & 0.343 & -0.113 & 0.487 \\
\hline BPF & -0.132 & 0.405 & -0.157 & 0.334 \\
\hline T2 LV & 0.131 & 0.407 & 0.056 & 0.732 \\
\hline SCF & -0.312 & 0.045 & -0.310 & 0.051 \\
\hline SCL & -0.254 & 0.361 & --- & -- \\
\hline
\end{tabular}

Abbreviations: EDSS = Expanded Disability Status Scale, T25FW = timed 25-foot walk, $\mathrm{WMF}=$ white matter fraction, $\mathrm{GMF}=$ grey matter fraction, $\mathrm{BPF}=$ brain parenchyma fraction, $\mathrm{SCF}=$ cervical spinal cord fraction, $\mathrm{T} 2 \mathrm{LV}=\mathrm{T} 2$ lesion volume, $\mathrm{SCL}=$ cervical spinal cord lesions. 
Table 7.4: Two multivariate regressions with a cord MTR metric among the independent variables. In the first regression the dependent variable is EDSS and in the second is T25FW $(* \mathrm{p}<0.05)$.

\begin{tabular}{|c|c|c|}
\hline & $\begin{array}{c}\text { EDSS } \\
\left(R^{2}=0.390\right)\end{array}$ & $\begin{array}{c}\text { T25FW } \\
\left(R^{2}=0.397\right)\end{array}$ \\
\hline Independent variables & \multicolumn{2}{|c|}{$\mathrm{p}$ value } \\
\hline Age & $0.006^{*}$ & $0.009^{*}$ \\
\hline Gender & 0.053 & $0.013^{*}$ \\
\hline T2LV & 0.078 & 0.752 \\
\hline SCF & 0.949 & 0.166 \\
\hline Brain volume & 0.464 & 0.128 \\
\hline Median whole cord MTR & 0.090 & 0.330 \\
\hline
\end{tabular}

Abbreviations: EDSS = Expanded Disability Status Scale, T25FW = timed 25-foot walk, $\mathrm{T} 2 \mathrm{LV}=\mathrm{T} 2$ lesion volume, $\mathrm{SCF}=$ cervical spinal cord fraction, $\mathrm{MTR}=$ magnetization transfer ratio.

Table 7.5: Two multivariate regressions with a cord MTsat metric among the independent variables. In the first regression the dependent variable is EDSS and in the second is T25FW $(* \mathrm{p}<0.05)$.

\begin{tabular}{|c|c|c|}
\hline & $\begin{array}{c}\text { EDSS } \\
\left(R^{2}=0.418\right)\end{array}$ & $\begin{array}{c}\text { T25FW } \\
\left(R^{2}=0.400\right)\end{array}$ \\
\hline Independent variables & \multicolumn{2}{|c|}{$\mathrm{p}$ value } \\
\hline Age & $0.002^{*}$ & $0.006^{*}$ \\
\hline Gender & 0.066 & $0.015^{*}$ \\
\hline T2LV & 0.117 & 0.845 \\
\hline SCF & 0.862 & 0.204 \\
\hline Brain volume & 0.637 & 0.161 \\
\hline Median whole cord MTsat & $0.034^{*}$ & 0.288 \\
\hline
\end{tabular}

Abbreviations: EDSS = Expanded Disability Status Scale, T25FW = timed 25-foot walk, $\mathrm{T} 2 \mathrm{LV}=\mathrm{T} 2$ lesion volume, $\mathrm{SCF}=$ cervical spinal cord fraction, $\mathrm{MTsat}=$ magnetization transfer saturation. 
Figure 7.1: MRI and MT maps from two MS patients of the brain and cervical spinal cord (with axial detail of a lesion area) illustrating the unreliable correlation between brain and cord involvement. (Study codes Patient A: CRT114127_000115; Patient B: GSK111009_000176). The top row (A) is a 31 years old woman with a history of RRMS with an EDSS score of 2. The patient shows a total brain lesion volume of $6.58 \mathrm{~mL}$ and a spinal cord lesion volume of $0.110 \mathrm{~mL}$. The bottom row (B) is a 42 years old woman with a history of RRMS with an EDSS score of 6. In this case the patient shows a total brain lesion volume of $29.44 \mathrm{~mL}$ but a low total spinal cord lesion volume of $0.060 \mathrm{~mL}$.

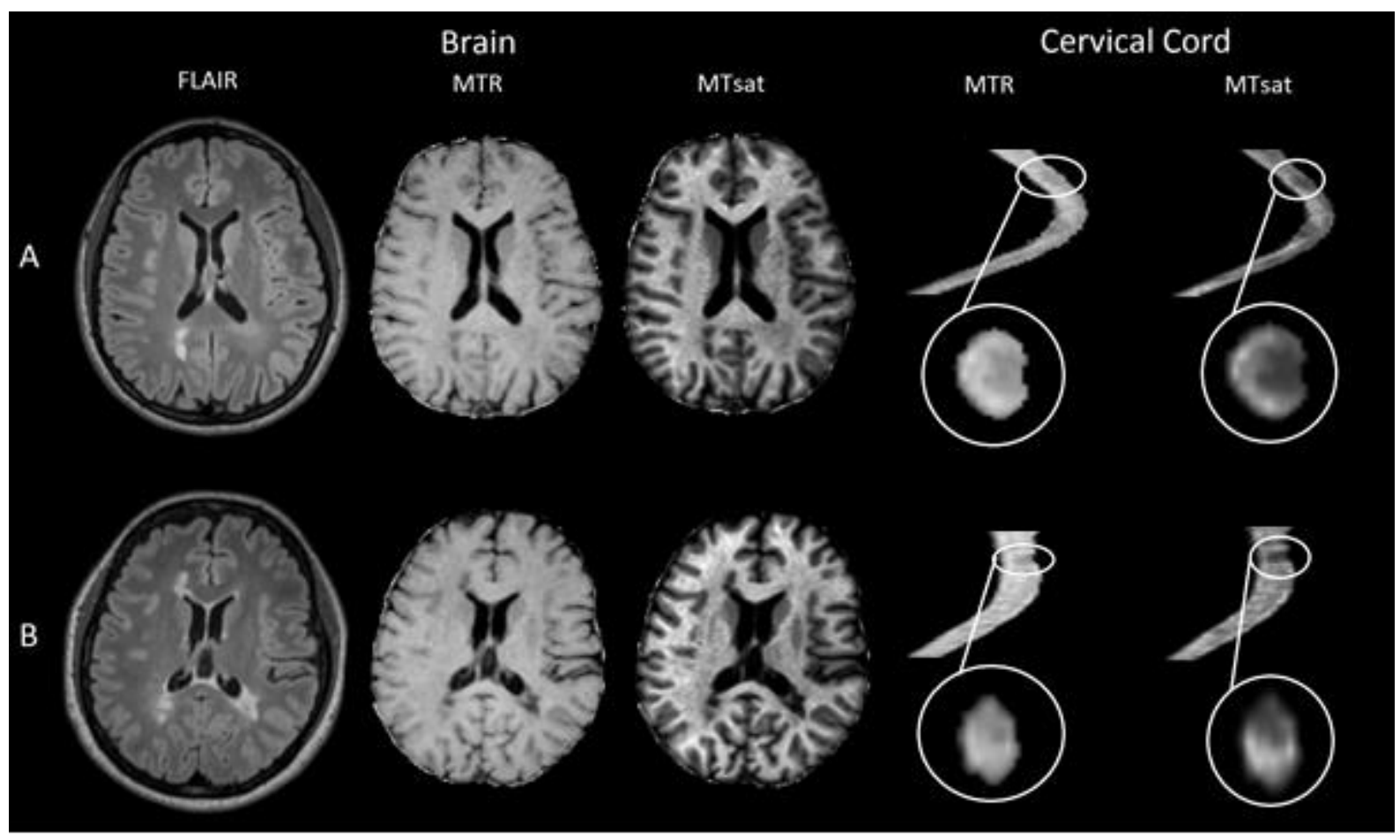

Abbreviations: $\mathrm{MT}=$ magnetization transfer, $\mathrm{MTR}=$ magnetization transfer ratio, MTsat $=$ magnetization transfer saturation, $\mathrm{MS}=$ multiple sclerosis, $\mathrm{RRMS}=$ relapsing remitting multiple sclerosis, EDSS = Expanded Disability Status Scale. 
Figure 7.2: Scatter plots of the cervical cord volume and the fractional volume against EDSS and T25FW.
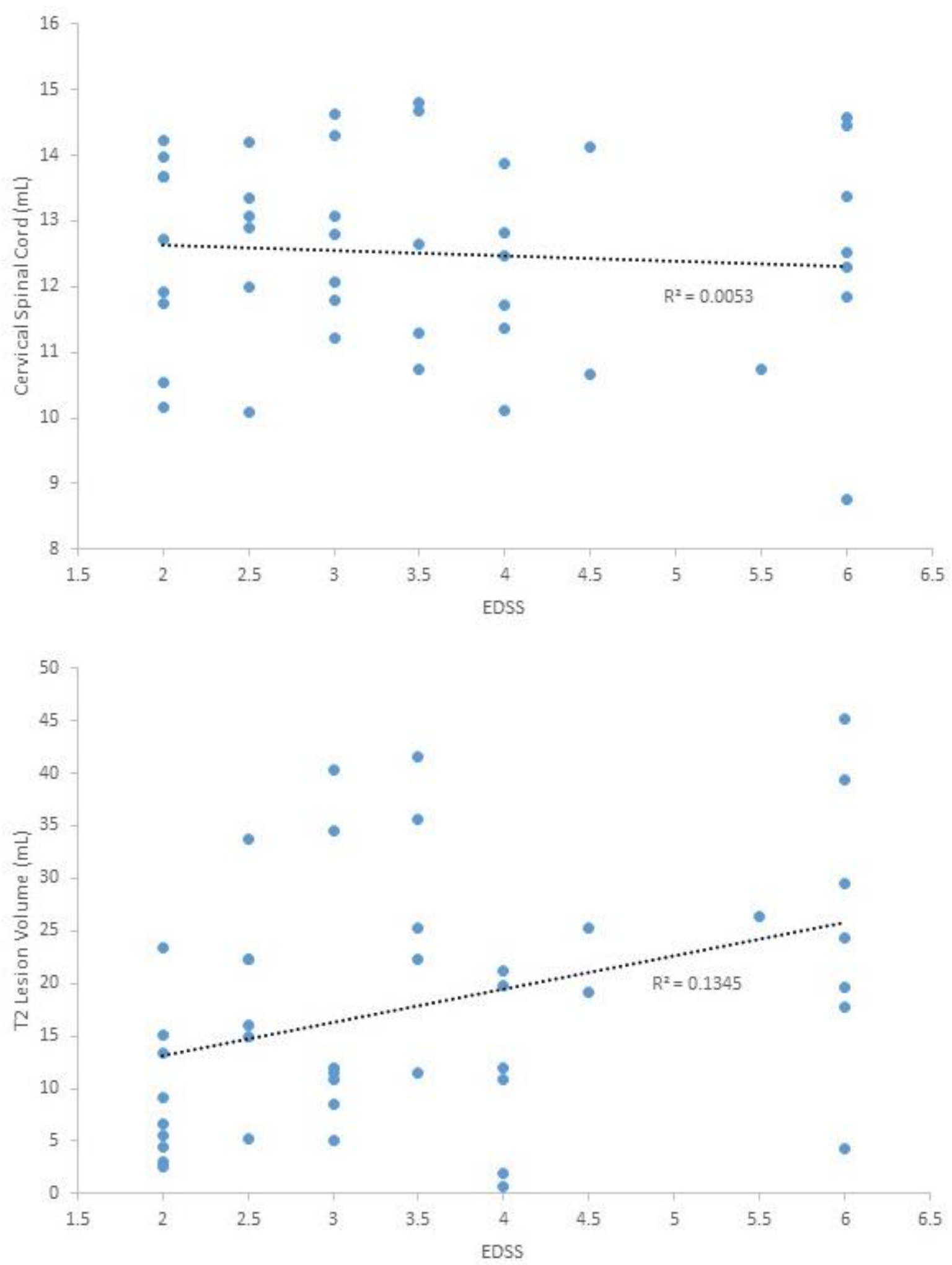

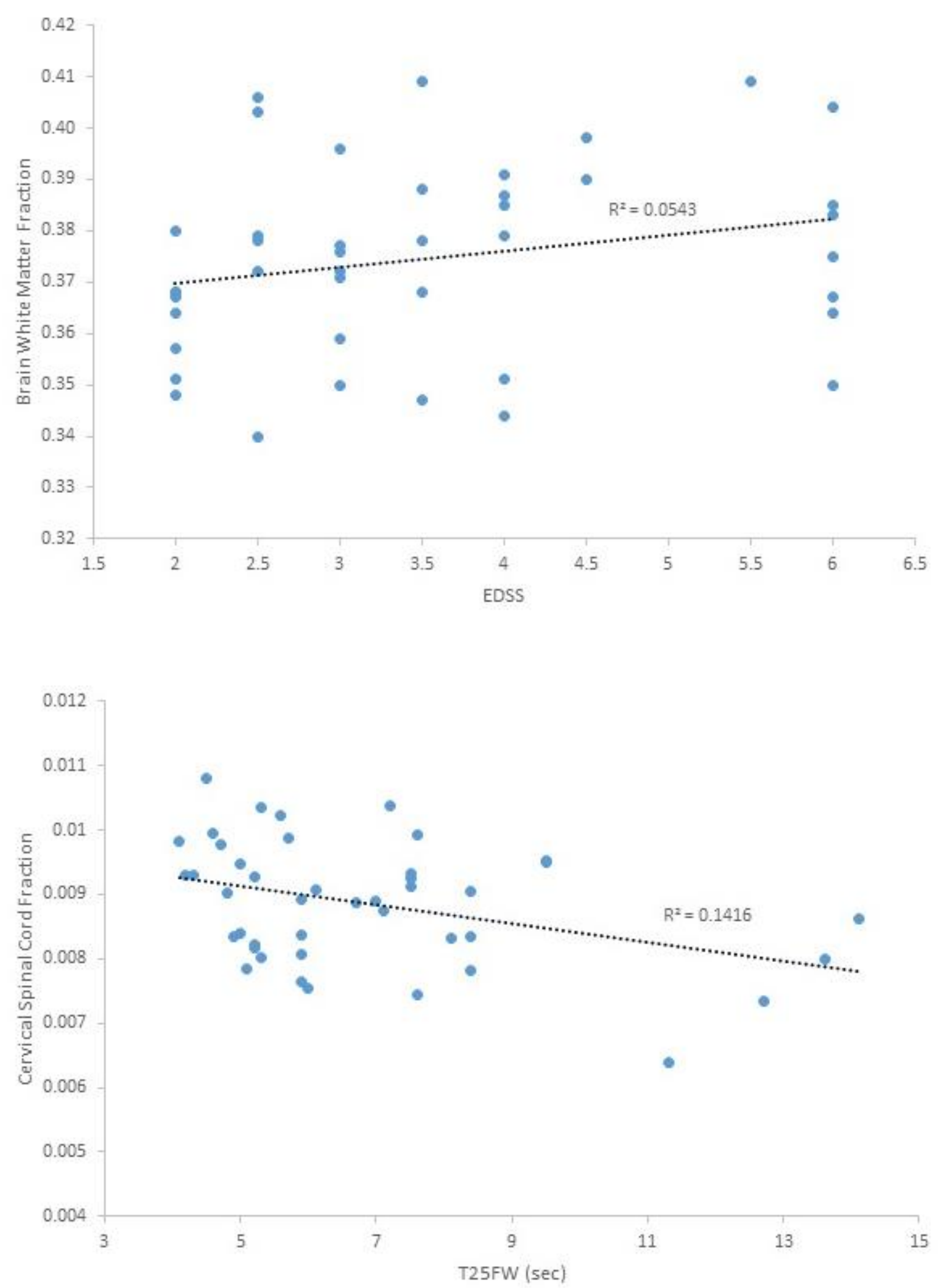

Abbreviations: EDSS = Expanded Disability Status Scale, T25FW = timed 25-foot walk. 
Figure 7.3: Scatter plot of the brain T2 lesion volume against the cervical spinal cord fraction.

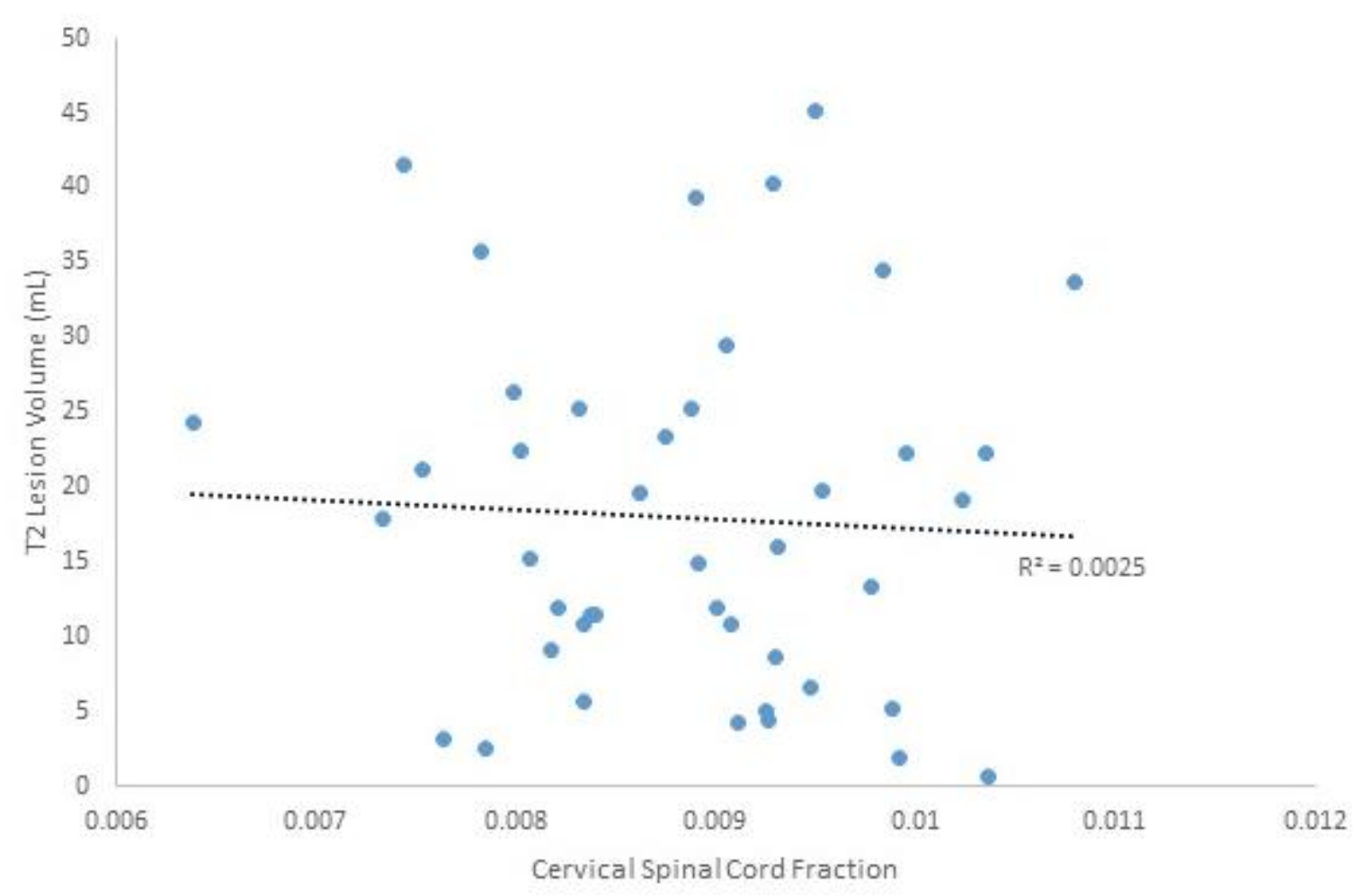




\section{Chapter 8}

\section{Conclusions}

\subsection{Achieving the objectives}

The main goal of this thesis was to measure magnetization transfer in the brain and the cervical cord in order to assess whether it can discriminate between disability levels in MS. MTsat achievements for those regions make of this quantitative magnetization transfer parameter an alternative to the wide used MTR. A complete database was acquired within the REMS project scanning patients at the Charing Cross Hospital and Hammersmith Hospital of London (UK) and enabling the selection of medium and large cohort of patients for this thesis.

The clinico-radiological paradox or disconnection between clinical and radiologic findings is frequent in MS and is particularly striking for studies of the spinal cord, where lesions might be expected to cause clinical impairment given the "eloquence" of the ascending and descending fibre tracts. While it may seem obvious that patients who develop new lesions are worse than those without new lesions, the association between clinical findings and radiological extent of involvement generally is limited and poor.

Normal appearing tissue in MS- at least by criteria of conventional MRI (without focal T2W lesions) - is microscopically abnormal. The quantitative MTsat appears to be more sensitive to abnormalities in the NAWM correlating with 
disability than is the standard MTR parameter. This quantitative parameter could enhance sensitivity to detect neuropathological changes that are clinically meaningful.

Increased water content results in slower longitudinal relaxation and tends to reduce MT. After an MT pulse has been applied, these effects amplify each other transiently, but they partially cancel each other in the steady state achieved with the FLASH imaging sequences widely employed in clinical scanners. This is a fundamental limitation within the MTR. The MTsat represents the effort to overcome this limitation. This parameter detects effects more specifically associated with the magnetisation saturation.

This thesis revealed that MTsat can be used to differentiate disability levels based on observations in the cervical cord as has previously been shown for MTR. After extracting the focal lesions, the NASC show significant correlations for both mean and median MTsat with just EDSS (which is heavily weighted toward spinal cord functions, such as ambulation). This work emphasised that, although the most visually evident imaging abnormality in MS by conventional MRI, focal T2 lesions do not correlate well with disability. Potentially relevant is the clinical observation that T2 spinal cord lesions are often clinically silent; asymptomatic spinal cord lesions have been reported in about $50 \%$ of patients with early $\mathrm{MS}^{131}$.

Although a minor effect in our hands, cervical cord atrophy study may reflect some anatomical considerations. The cervical volume may be an accurate correlate of upper motor neuron and trunk motor function as it contains all descending corticospinal fibers destined for motor targets in the trunk, arms and legs. Also, the cervical cord is a crossroads for all cerebrospinal descending and spinocerebral ascending pathways. Thus, as compared to other brain and cord locations, damage to cervical cord disproportionately affects lots of functions. 


\subsection{Future challenges}

After studying the potential of the MTsat in a region of the brain with such an interest in terms of silent lesions and microstructural changes caused by the course of the disease like in the case of the NAWM, other regions of interest could be potentially target for further researches. This is the case of the dirty appearing white matter (DAWM) which refers to brain regions of intermediate signal intensity between those of focal lesions and NAWM on T2W images. Such an abnormality tends to affect more frequently the deep WM, especially around the ventricles. During the course of this thesis some attempts to delineate this region were made, but defining the extent of DAWM is, however, a challenging task, because signal intensity changes associated with it are difficult to clearly separate from the surrounding tissues. The pathologic substrates of DAWM abnormalities have been investigated only recently and was found that a loss of myelin phospholipids as well as axonal reduction whose extents are intermediate between those discrete lesions and NAWM.

The MTsat is limited at high field strengths by specific absorption rate (SAR, the amount of energy per second absorbed per kilogram of body mass) which limits the acquisition speeds. In order to acquire MT data at higher field, such as $7 \mathrm{~T}$ and 9.4T magnets, changes should be developed in how the MT data is acquired. New protocols can be made in order to measure the size of MT exchanging pools, based on recent methods for mapping the relative density of the macromolecular protons. This is the case of a new method, where labelled spins by a stimulated echo, serves as an intrinsic indicator ${ }^{132}$. Following the free distribution of this indicator, the macromolecular pool size is determined using indicator dilution theory (after adding an indicator to the free protons pool, an exchange process will cause a new steadystate concentration. The fractional size of the restricted pool can be calculated from 
the change in the indicator concentration). This approach allows the SAR to be kept at a very low level. 


\section{Binary spin bath model and MTsat}

\section{A1. Modelling MT}

This model consists of two pools (Figure A.1): a free (bulk water) and a restricted (macromolecular) pool. Both pools represent longitudinal magnetization (unshaded) and other magnetization (shaded), which may be transverse or saturated. It is assumed that each compartment is associated with an intrinsic longitudinal relaxation time $T 1$, shown as the relaxation constants $R_{f}$ (free pool) and $R_{r}$ (restricted pool). The equilibrium magnetization of the free pool is called $M_{0 f}$ and the restricted pool is $M_{0 r}$.

The restricted pool is shown smaller than the free pool because its magnetization at equilibrium is only a few percent of the free pool for an agar gel system.

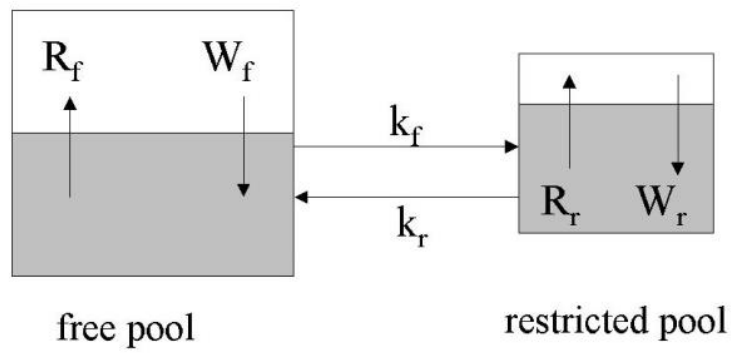

Figure A.1: Two pools model for magnetization transfer between free and restricted protons. 
Two exchange rate constants $k_{f}$ and $k_{r}$ are added to characterize the forward and reverse exchange process between the free and restricted pools. When an off resonance RF pulse is applied, saturation of the pools takes place. This saturation depends on the RF pulse characteristics and the absorption line shape of the restricted pool. This rate of loss of longitudinal magnetization is shown as $W_{f}$ and $W_{r}$ for the free and restricted pool respectively.

The evolution of the magnetization of this system is described by a set of coupled differential equations. The free pool is described by a modified version of the Bloch equations that includes saturation due to the MT pulse and exchange of the longitudinal magnetization:

$$
\begin{gathered}
\frac{d M_{x f}}{d t}=-\frac{M_{x f}}{T_{x f}}-\Delta M_{y f}-\operatorname{Im} \omega_{1}(t) M_{z f} \\
\frac{d M_{y f}}{d t}=-\frac{M_{y f}}{T_{z f}}-\Delta M_{x f}-\operatorname{Re} \omega_{1}(t) M_{z f} \\
\frac{d M_{z f}}{d t}=R_{1 f}\left(M_{o f}-M_{z f}\right)-k_{f} M_{z f}+k_{r} M_{z r}+\operatorname{Im} \omega_{1}(t) M_{z f}-\operatorname{Re} \omega_{1}(t) M_{y f} \\
\frac{d M_{z x}}{d t}=R_{1 r}\left(M_{o r}-M_{z r}\right)-k_{r} M_{z r}+k_{f} M_{z f}-W_{r} M_{z r}
\end{gathered}
$$

These equations can be written in matrix form:

$$
\frac{d M(t)}{d t}=\boldsymbol{A}(t) \boldsymbol{M}(t)+\boldsymbol{B} \boldsymbol{M}_{0}
$$

where $\mathbf{M}$ is the magnetization vector, $\boldsymbol{M}_{0}$ is the fully relaxed state of the magnetization and $\mathbf{A}$ and $\mathbf{B}$ are matrices with the coefficients of the equations A.1A.4. The subscripts $f$ and $r$ denote the free and restricted pools and the subscripts $x, y$ and $z$ denote the components of the magnetization vector. The subscripts 1 and 2 
denote the longitudinal and transverse relaxation of the magnetization. $\mathrm{F}$ is the ratio of the pool sizes or the fractional pool size:

$$
F=\frac{M_{0 r}}{M_{0 f}}
$$

In equilibrium: $k_{r}=k_{f} / F$. The transition rate $W_{r}$ of the restricted pool under $\mathrm{CW}$

irradiation is given by:

$$
W_{r}=\pi \omega_{1 C W}^{2} G(\Delta)
$$

$\mathrm{G}$ is the absorption line shape of the restricted pool, which depends on the tissue and the frequency offset ( $\Delta$ is the frequency offset of the CW irradiation relative to $\omega_{0}$ ) and $\omega_{1 C W}$ is the frequency of the continuous wave associated with its power $\left(\omega_{1 C W}=\gamma B_{1 C W}\right)$. A Gaussian line shape has been found appropriate for gels:

$$
G(\Delta)=\frac{T_{2 \pi}}{\sqrt{2 \pi}} e^{-2\left(\pi \Delta T_{2 \pi}\right)^{2}}
$$

Brain tissue can be described by a super-Lorentzian line shape:

$$
G(\Delta)=T_{2 r} \int_{0}^{1} \frac{1}{\left|3 u^{2}-1\right|} \exp \left(-2\left(\frac{2 \pi \Delta T_{2 r}}{3 u^{2}-1}\right)\right) d u
$$

It was proved that the equation A.7 can also be used in the case of RF pulses ${ }^{133}$ :

$$
W_{r}=\pi \omega_{1}^{2}(t) G(\Delta)
$$

Where $\Delta$ is the frequency offset of the pulse relative to $\omega_{0}$.

The CW case gives a simplification of the Bloch equations A.1-A.4 because the MT pulse is not time dependent and the equations can be solved analytically. Therefore, an equation for the magnetization as a function of the frequency offset can be written: 


$$
M_{z f}=\frac{M_{0 f}\left(R_{1 r} k_{f}+R_{1 r} R_{1 f}+R_{1 f} k_{r}+W_{r} R_{1 f}\right)}{\left(R_{1 f}+\left(\frac{\omega_{1 C W^{T}} T_{2 f}}{1+\left(\omega T_{2 f}\right)^{2}}\right)+k_{f}\right)\left(R_{1 r}+W_{r}+k_{r}\right)-k_{f} k_{r}}
$$

MT measurements were made of an agar gel system, avoiding the assumption that the macromolecular pool was completely saturated and that the water pool was unaffected $^{134}$. These measurements showed a decreased signal due to both direct saturation of water protons and MT. The $T_{1}$ and $T_{2}$ values of the agar samples were used to calculate the decreased signal from direct saturation making possible to determine the amount of MT.

The equations A.1-A.4 do not include the possibility of MT exchange between the pools due to the transverse magnetization. The magnitude of such an exchange is expected to be very small because the very short $T_{2}$ relaxation time of the macromolecular pool. It was confirmed that the effect is negligible ${ }^{133}$.

The free water should be represented by at least two pools with different relaxation behaviour and some exchange between them, also tissue water exhibits multiple relaxation times and it has been shown that each of these relaxation species experiences its own magnetization transfer ${ }^{63}$. However, in the case of agar gel, the model is a good description of the transfer processes.

The model for the macromolecular pool is not precise either because we should also take into account the dipolar coupling. These dipolar coupling is due to intermolecular forces which arise in solids or semisolids and produces an extra splitting of the energy levels beside the Zeeman effect. 


\section{A.2 Pulsed irradiation and MTsat}

The effect of both the MT pulse and the excitation pulse on the free pool is modelled as an instantaneous fractional saturation of the longitudinal magnetization $S_{f}{ }^{135}$. The

Pike's model includes these approximations: instantaneous saturation of the free pool, free precession of the free pool and CW irradiation of the restricted pool. The relation of the magnetization after the pulses $M_{z f}^{+}$and before the pulses $M_{z f}^{-}$is given by:

$$
M_{z f}^{+}=S_{1 f} S_{2 f} M_{z f}^{-}=S_{f} M_{z f}^{-}
$$

The fraction of $M_{z f}$ that remains after the MT pulse is $S_{1 f}$, and the fraction of $M_{z f}$ that remains after the excitation pulse is $S_{2 f}$. The effect on the magnetization can be written like:

$$
\left[\begin{array}{l}
M_{z f}^{-} \\
M_{z r}^{-}
\end{array}\right]=\left[\begin{array}{cc}
s_{f} & 0 \\
0 & S_{r}
\end{array}\right]\left[\begin{array}{l}
M_{z f}^{+} \\
M_{z r}^{+}
\end{array}\right]
$$

In this matrix equation, $\boldsymbol{M}^{+}=\boldsymbol{S M}^{-}, S_{f}$ and $S_{r}$ denote the fractions of longitudinal magnetization that remains after the pulses for the free and restricted pools. The equations A.3 and A.4 can be simplified when the effect of a continuous wave excitation during TR of equivalent total energy is taken as the shaped MT pulse:

$$
\begin{gathered}
\frac{d M_{z f}}{d t}=R_{1 f}\left(M_{0 f}-M_{z f}\right)-k_{f} M_{z f}+k_{r} M_{z r} \\
\frac{d M_{z r}}{d t}=R_{1 r}\left(M_{0 r}-M_{z r}\right)-k_{r} M_{z r}+k_{f} M_{z f}-W_{r} M_{z r}
\end{gathered}
$$

The transverse components disappear completely through relaxation and spoiling at the end of a pulse period. The equations A.14 and A.15 can be expressed in matrix form: 


$$
\left[\begin{array}{c}
\frac{d M_{z f}(t)}{d t} \\
\frac{d M_{z r}(t)}{d t}
\end{array}\right]=\left[\begin{array}{cc}
-R_{1 f}-k_{f} & k_{r} \\
k_{f} & -R_{1 r}-k_{r}-W_{r}
\end{array}\right]\left[\begin{array}{cc}
R_{1 f} & 0 \\
0 & R_{1 r}
\end{array}\right]\left[\begin{array}{c}
M_{0 f} \\
M_{0 r}
\end{array}\right]
$$

The general solution for this equation is:

$$
\boldsymbol{M}(t)=e^{-A_{C W} t} \boldsymbol{M}(0)+\left[\boldsymbol{I}-e^{-A_{C W} t}\right] \boldsymbol{M}_{C W}^{S S}
$$

The $\boldsymbol{M}_{C W}^{S S}$ matrix depicts the magnetization in the steady state and equals the magnetization due to a long period of continuous wave irradiation of the restricted pool. When the effect of the instantaneous saturation of the free pool is combined with the continuous wave saturation of the restricted pool, the magnetization is:

$$
\boldsymbol{M}(t)=e^{-A_{C W} t} \boldsymbol{M}^{-}+\left[\boldsymbol{I}-e^{-A_{C W} t}\right] \boldsymbol{M}_{C W}^{S S}
$$

The steady state signal in Pike's model is obtained for $\tau=T R$ and a readout by $\sin \alpha$.

It can be written in formal analogy to the Ernst equation:

$$
S=A \sin \alpha \frac{1-\exp \left(-R_{1} T R\right)}{1+\left[S_{f}+T\left(S_{T}-S_{f}\right) \frac{1-\exp \left(-R_{T} T R\right)}{1-\left(1-S_{T}\right) \exp \left(-\left(R_{T}+R_{1}\right) T R\right)}\right] \exp \left(-R_{1} T R\right)}
$$

Where $T=k_{f} /\left(k_{f}+k_{r}\right)$ and $R_{T}=k_{f}+k_{r}$. After linear approximation and by comparison with the equation 4.11 , it can be written:

$$
\text { MTsat } \cong T\left(S_{r}-S_{f}\right) \frac{1-\exp \left(-R_{T} T R\right)}{1-\left(1-S_{r}\right) \exp \left(-\left(R_{T}+R_{1}\right) T R\right)}+S_{f}
$$

The MTsat is proportional to saturation terms and shows minor influence of relaxation. The MTsat is expected to increase with the energy of the MT pulse and decrease for larger offset frequencies. 


\title{
APPENDIX B
}

\section{A Comparison of Magnetization Transfer Methods to Assess Brain and Cervical Cord Microstructure in Multiple Sclerosis}

Alfonso Lema, Courtney Bishop, Omar Malik, Miriam Mattoscio, Rehiana Ali, Richard Nicholas, Paolo A. Muraro, Paul M. Matthews, Adam D. Waldman, Rexford D. Newbould

Fromthe Dixision of Brain Sciences, Department of Medicine, Imperial College London, London, UK (AL, OM, MM, RA, RN, PAM, PMM); Imanova Centre for Imaging Sciences, London, UK (CB, RDN); and Department of Imaging, Imperial College Healthcare NHS Trust, London, UK (ADW)

\begin{abstract}
A B S T R A C T
BACKGROUND: Demyelination is a core pathological feature of multiple sclerosis (MS) and spontaneous remyelination appears to be an important mechanism for repair in the disease. Magnetization transfer ratio imaging (MTR) has been used extensively to evaluate demyelination, although limitations to its specificity are recognized. MT saturation imaging (MTsat) removes some of the T1 dependence of MTR. We have performed a comparative evaluation of MTR and MTsat imaging in a mixed group of subjects with active MS, to explore their relative sensitivity to pathology relevant to explaining clinical outcomes.

METHODS: A total of 134 subjects underwent MRI of their brain and cenvical spinal cord. Isotropic 3-dimensional pre- and postcontrast T1-weighted and T2-weighted fluid-attenuated inversion recovery (FLAIR) volumes were segmented into brain normal appearing white matter (NAWM), brain WM lesions (WML), normal appearing spinal cord (NASC), and spinal cord lesions. Volu mes and metrics for MTR and MTsat histograms were calculated for each region.

RESULTS: Significant Spearman correlations were found with the Expanded Disability Status Scale and timed 25-foot walk for the whole brain and WML MTR, but not in that from the NAWM or any cervical spinal cord region. By contrast, the MTsat was correlated with both disability metrics in all these regions in both the brain and spine.

CONCLUSIONS: This study extends prior work relating atrophy and lesion load with disability, by characterization of MTsat parameters. MTsat is practical in routine clinical applications and may be more sensitive to tissue damage than MTR for both brain and cervical spinal cord.
\end{abstract}

Keywords: Magnejzation transfer, MRI, multiple sclerosis, atrophy, spinal cord

Acceptance: Received May 9, 2016, and in revised form June 25, 2016. Accepted for publication June 26, 2016.

Cor respondence: Address correspondence to Prof. Paul M. Matthews, Edmond and Lilly Safra Chair, Head, Division of Brain Sciences, Imperial College London, Burlington Danes Building, DuCane Road, London WC12 ONN, UK. E-mail: p.matthews @imperial.ac.uk.

Paul M. Matthews, Adam D. Waldman, and Rexford D. Newbould are joint senior authors.

Acknowledgments: This work was supported by the Imperial College Healthcare Trust Biomedical Research Centre, Medical Research Council (Ref. No. G0800679), GlaxoSmithKline and the EC. PMM acknowledges personal support from the Edmond J Safra Foundation and Lily Safra.

Disclosure: Dr. Muraro has received travel support and speaker honoraria from Bayer HealthCare, Bayer Pharma, Biogen Idec, Merck-Serono, Sanofi Aventis, and Novartis. Prof. Matthews and Dr. Newbould were employees of GlaxoSmithKline Research and Development at the time of the study. Prof. Matthews has received research or educational funding or has had honoraria paid to Imperial College from Biogen, Novartis, IXICO, and Transparency Life Sciences. Prof. Waldman has received educational grant funding or speaker honoraria from Bayer HealthCare, Biogen, and Novartis.

J Neuroimaging 2016;00:1-6.

DOI: $10.1111 /$ jon. 12377

\section{Introduction}

Multiple sclerosis (MS) produces both inflammatory and degenerative pathology in the brain and spinal cord. Conventional magnetic resonance imaging (MRI) of the brain is highly sensitive for detecting focal inflammatory lesions in patients with MS. Assessments of the burden of brain T2 lesions are used to monitor evolution of MS clinically. However, only modest correlations between brain MRI lesion load and clinical disability have been found. ${ }^{1}$ One explanation for this is that comprehensive detection of lesions in spinal cord MRI still is more challenging than for the brain although occurrence of MS lesions in the cord is believed to give rise to clinical symptoms more often than lesions located in the brain. ${ }^{2,3}$ The assessment of MS pathology using T2-weighted images is also limited by the poor specificity of this technique to the heterogeneous substrates of MS lesions, ${ }^{4}$ and by its inability to provide information about the microscopic changes occurring in the normal appearing white matter (NAWM) ${ }^{5}$ Perhaps, most important, however, is that substantial neuronal and axonal loss are found in the brain and spinal cord, in addition to demyelination. ${ }^{6-10}$

Magnetization transfer imaging (MTI) provides quantitative metrics sensitive to microstructural changes reflecting some neurodegenerative aspects of MS pathology that, ${ }^{11,12}$ in conjunction with conventional MRI measures, allow the combined macroscopic and microscopic disease burdens to be estimated. ${ }^{13}$ More specifically, MTI reflects the exchange of magnetization between freely mobile protons and those associated with macromolecules in myelin or axonal membranes. ${ }^{14}$ The MT ratio (MTR) is strongly correlated with the percentage of residual myelin and axons present in MS lesions and NAWM. ${ }^{15}$ However, conventional MTR is sensitive to biophysical parameters that may limit its precision, particularly in the spinal cord. 
The alternative approach of quantitative MT saturation (MTsat) offers a measure that, unlike MTR, is minimally affected by $\mathrm{T} 1$ relaxation and is less sensitive to $\mathrm{B} 1$ inhomogeneities. WM to gray matter (GM) contrast in MTsat maps is increased relative to that in MTR maps. ${ }^{16}$ MTsat thus may enhance insights into clinicopathological correlations related to changes in the cervical cord in MS relative to the MTR.

The goals of this study are to compare MTR and MTsat measures across an MS population and to analyze their capability to discriminate between disability levels when they are applied to the brain and cervical spinal cord of a cohort of 134 patients. Correlations between MTR and MTsat histogram metrics of the brain and the cervical cord and disability were explored.

\section{Methods}

Patients

The cohort of patients formed part of the Patient Research Cohort Rapidly Evolving Multiple Sclerosis Study (PRC-REMS), which had the approval of the London-Chelsea Regional Ethics Committee (NCT01044576) and was conducted in accordance with the principles of Good Clinical Practice (GCP). MRI scans of the brain and cervical spine for 134 patients were available for this study. The degree of disability was estimated according to the Kurtzke's Expanded Disability Status Scale (EDSS) and the timed 25-foot walk (T25FW) scale from the Multiple Sclerosis Functional Composite.

\section{MRI Acquisition}

The study was performed on a 3T clinical MR system (Magnetom Verio, Siemens Medical Solutions, Erlangen, Germany). A combined head and neck radiofrequency (RF) coil system consisting of a 12-channel phased array head coil and four-channel phased array neck coil was used for reception. The system body coil was used for all excitation and MT $\mathrm{RF}$ pulses, and no repositioning occurred between sequences. Pre- and postcontrast T1-weighted 3-dimensional (3D) Magnetization Prepared Rapid Gradient Echo (MPRAGE) volumes were acquired similarly to the Alzheimer's Disease Neuroimaging Initiative-recommended parameters except $1 \mathrm{~mm}$ isotropic resolution, ${ }^{17}$ parallel imaging (PI) factor of 2 , and $256 \times 192 \mathrm{~mm}$ field of view (FOV) in $5 \mathrm{~m}: 21$ seconds in the brain. A .6 $\times .6 \times$ $2.0 \mathrm{~mm}$ resolution and $160 \times 160 \times 160 \mathrm{~mm}$ FOV were used in the cervical spinal cord in $3 \mathrm{~m}: 43$ seconds. A physician injected gadolinium contrast (Gadoterate meglumine, Dotarem, Guerbet, $.1 \mathrm{mmol} / \mathrm{kg}$ ) 5 minutes before the acquisition of the final image volumes: the postcontrast MPRAGE volumes of the brain and c-spine. All other images were acquired before the contrast injection. T2-weighted fluid-attenuated inversion recovery (FLAIR) volumes were acquired with $1 \mathrm{~mm}$ isotropic resolution and 160 sagittal sections in a single $3 \mathrm{D}$ slab, $250 \times$ $250 \mathrm{~mm}$ FOV, PI factor of 2 , inversion time (TI) $1,800 \mathrm{~ms}$, repetition time (TR) 5 seconds, and echo time (TE) $395 \mathrm{~ms}$, in $5 \mathrm{~m}: 52$ seconds in the brain. A 3D fast low-angle shot (FLASH) sequence with $1 \mathrm{~mm}$ isotropic resolution, 192 sagittal partitions, and $256 \times 240$ FOV was employed in the brain (with a readout in the head-feet direction with $600 \mathrm{~Hz} /$ pixel bandwidth to reduce susceptibility artifacts). Six monopolar gradient echoes equally spaced between TE of 1.95 and $11.7 \mathrm{~ms}$ were acquired during a TR of $27 \mathrm{~ms}$. A PI factor of 2 was used for the GRAPPA parallel acquisitions. A flip angle of $5^{\circ}$ was chosen for the proton density-weighted (PD-w) dataset, meanwhile another was MT-weighted by a band selective RF pulse of Gaussian shape with a duration of $12.24 \mathrm{~ms}, 2.2 \mathrm{kHz}$ frequency offset, and nominal $540^{\circ}$ flip angle, in $7 \mathrm{~m}: 20$ seconds. A T1-weighted volume, similar to the PD-w but with a TR of $15 \mathrm{~ms}$ and a flip angle of $18^{\circ}$, was also acquired in $4 \mathrm{~m}: 05$ seconds. A similar set of three contrasts at $1 \mathrm{~mm}$ isotropic resolution was acquired in the cervical spinal cord, but with a smaller FOV of $192 \times 156 \times$ $120 \mathrm{~mm}$. The PD-w and MT-weighted acquisitions used a TR of $26 \mathrm{~ms}$, requiring $4 \mathrm{~m}: 59$ seconds each. The T1-weighted volume used a TR of $13 \mathrm{~ms}$ and flip angle of $15^{\circ}$. Other parameters remained the same as the brain acquisitions.

\section{Data Analysis}

MTR maps were produced on a voxel by voxel basis according to the equation:

$$
M T R=100 \frac{M_{0}-M_{s}}{M_{0}}
$$

where $M_{s}$ and $M_{0}$ are signal intensities with application of saturation pulse and without respectively. MTR values are reported in percent units (p.u.). The MTsat parameter was calculated by inserting the estimated $A_{a p p}$ and $T_{1 a p p}$ values in the approximate signal equation for the MT FLASH experiment. ${ }^{18}$ The MTsat parameter represents the additional percentage reduction in the steady-state FLASH signal caused by the saturation effect of a single MT pulse. It is calculated from this equation:

$M T_{o n_{P D w}}=A_{\alpha p p} \alpha\left(T R / T_{1 \alpha p p}\right) /\left(T R / T_{1 \alpha p p}+\alpha^{2} / 2+M T s a t\right)$

Using $A_{\alpha p p}$ and $T_{1 a p p}$ obtained from these equations:

$$
\begin{gathered}
A_{a p p}=\frac{\left(2 T R_{P D w} / \alpha_{P D w}^{2}-2 T R_{T 1 w} / \alpha_{T 1 w}^{2}\right)}{\frac{2 T R_{P D w}}{\alpha_{P D w} M T f_{P D w}}-\frac{2 T R_{T 1 w}}{\alpha_{T 1 w} M T o f f_{T 1 w}}} \\
T_{1 a p p}=\frac{\left(M T o f f_{P D w} / \alpha_{P D w}-M T o f f_{T 1 w} / \alpha_{T 1 w}\right)}{M T o f f_{P D w} \alpha_{P D w} / 2 T R_{P D w}} \\
-M T o f f_{T 1 w} \alpha_{T 1 w} / 2 T R_{T 1 w}
\end{gathered}
$$

MTsat has been shown to be insensitive to inhomogeneities in RF transmit field and receive fields. ${ }^{16}$ Unlike MTR, the MTsat is not affected by $\mathrm{T} 1$ relaxation.

SIENAX from FSL was used on T1-weighted images in the extraction of the brain and segmentation of GM and WM using a partial volume of threshold of .70 and estimation of their volumes. ${ }^{19}$ The cervical cord, brain WM lesions, and cervical spinal cord lesions were extracted manually with Analyze 11.0 (AnalyzeDirect/Mayo Clinic, KS). For each region, intensity distribution maps for segmented MTsat and MTR were calculated by Matlab (Mathworks Natick, MA) and histograms were generated for each subject with bins of width .25 p.u. ${ }^{20}$ The histogram median and height of the highest peak were recorded. Linear coregistration of datasets was performed using FLIRT. ${ }^{21}$

Volumes were normalized with intracranial volume (ICV) to reduce interindividual variation. Intersubjects coefficients of variation $(\mathrm{CoV})$ were calculated:

$$
\mathrm{CoV}=\mathrm{SD} / \text { mean } \times 100 \%
$$


Spearman rank-order correlation coefficient and Spearman's partial correlations (controlling for age, gender, and disease duration) assessed associations between volumes, MTR and MTsat histogram median and peak height, and both EDSS and T25FW.

Mixed models with patient as a random factor were calculated after the imputation of missing values. They determined the correlation between magnetization transfer histogram metrics and the volumes (segmented lesions, brain, and cord) with disability scales EDSS and T25FW. Parameter estimates and marginal means with $95 \%$ confidence intervals were presented.

A two tailed $P$ value less than .05 was considered significant and corrected thresholds for $P$ were applied for multiple comparisons. All statistical analyses were performed with SPSS for Windows (v. 17, SPSS Inc., Chicago, IL) except the comparison of correlations, which was done with the cocor package. $^{22}$

\section{Results}

Subjects included people with both relapsing-remitting MS (RRMS) and secondary progressive MS (SPMS) (Table 1). T1 lesions or T2 hyperintensities in the FLAIR volumes were identified in 121 cases. Cervical T2 lesions were identified in 19 subjects. 121/123 datasets met blinded image QC criteria.

Manual spinal cord segmentation was performed in 52 subjects. The MTsat maps showed higher contrast between WM, GM, and cerebrospinal fluid (CSF) relative to the MTR maps. The average histograms for the whole brain and for WM, GM, and CSF for the cohort of patients illustrate this (Figs 1A and B); the MTR histogram did not show a clear mode for the CSF. The MTsat peak height in the brain parenchyma was correlated with the MTR peak height $\{r=.590 ; P<.001\}$. This was not found for $\operatorname{CSF}(r=.122 ; P=.188)$. Segmentation of the spinal cord into $\mathrm{WM}$ and GM was not performed for tissue-specific analyses in the spinal cord.

Both whole-brain MTR and MTsat were correlated with median WM lesion (WML) volumes $(r=.654, P<.001$ for MTR and $r=.491, P<.001$ for MTsat), and their comparison showed they were significantly different $(z=2.892 ; P=$ .004). Significant correlations were also found between both magnetization transfer and clinical disability measures (EDSS and T25FW) (Table 2). For MTsat, significant correlations were

Table 1. Summary of Clinical Characteristics of Patients Involved in the Study

\begin{tabular}{lccc}
\hline & All & Brain & C-Spine \\
\hline Subjects & 134 & 121 & 52 \\
RRMS & 128 & 115 & 49 \\
SPMS & 6 & 6 & 3 \\
Age (years) & 39 & 39 & 38 \\
& $(25-66)$ & $(25-66)$ & $(28-59)$ \\
Gender (F/M) & $107 / 27$ & $97 / 24$ & $41 / 11$ \\
Disease duration (years) & $3(0-16)$ & $3(0-16)$ & $2(0-13)$ \\
EDSS & $3.5(2.0-6.0)$ & $3.5(2.0-6.0)$ & $3.5(2.0-6.0)$ \\
T25FW (seconds) & $6.0(1.3-15.3)$ & $5.9(1.3-15.3)$ & $6.1(4.1-14.1)$ \\
Relapses within last year & $2(0-5)$ & $2(0-5)$ & $2(0-4)$ \\
\hline
\end{tabular}

Median (range) values are shown for: age, disease duration, EDSS, T25FW, and relapses within last year. RRMS = relapsing reemitting multiple sclerosis; SPMS = secondary progressive multiple sclerosis; EDSS = Expanded Disability Status Scale; T25FW = timed 25-foot walk.

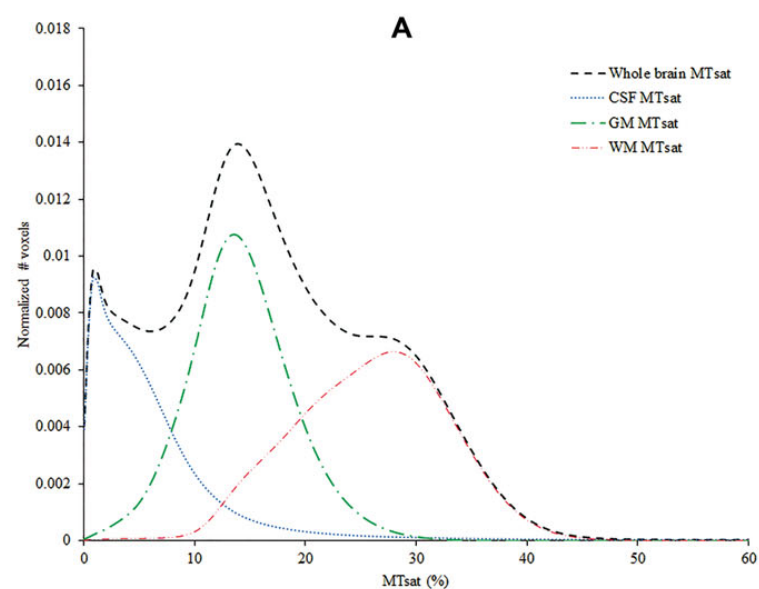

B

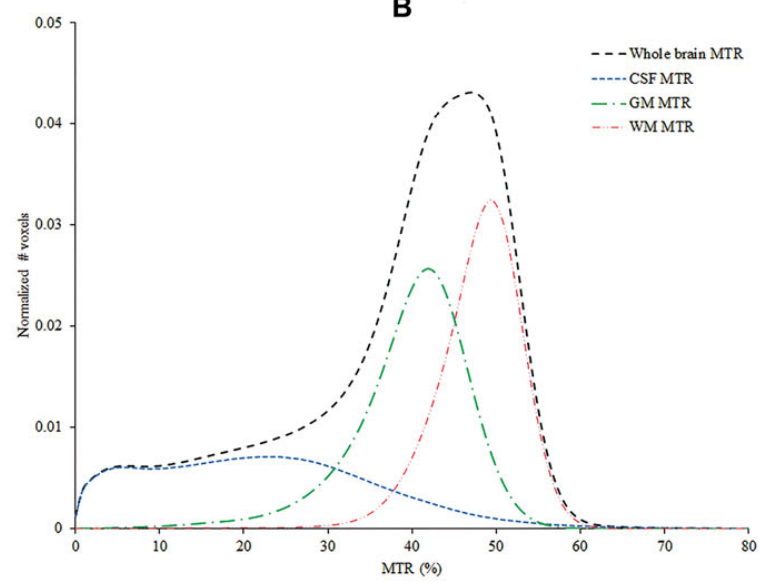

C

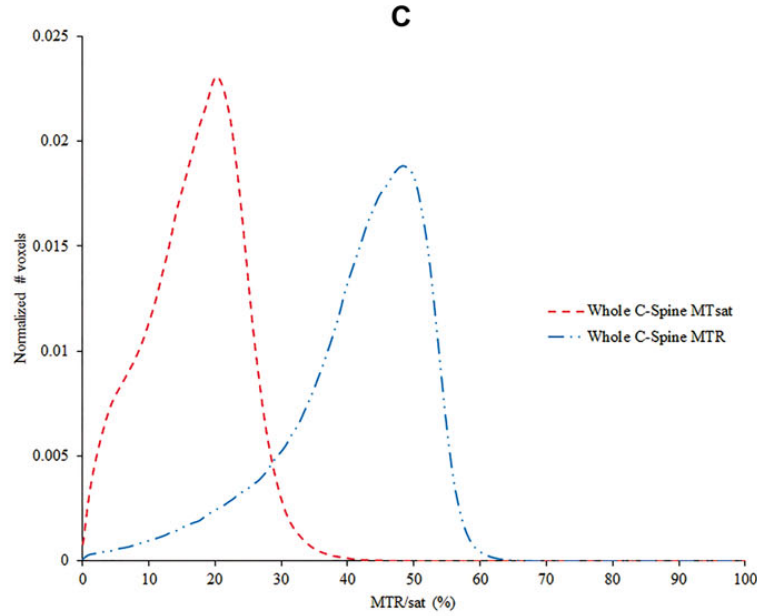

Fig 1. Average brain histograms across all subjects of MTsat (A) and MTR (B). The whole-brain MTR histogram is unimodal, while the MTsat is multimodal, as a result of the increased contrast between WM and GM. C-spine histograms (C) of MTR and MTsat show similar differences as in the brain. The spinal cord was not further segmented into gray and white matter. MTR = magnetization transfer ratio; MTsat = magnetization transfer saturation; $\mathrm{WM}=$ white matter $\mathrm{GM}=$ gray matter; $\mathrm{CSF}=$ cerebrospinal fluid . 
Table 2. Spearman Rank-Order Correlation Coefficients with Both Disability Scales for MTR and MTsat in the Brain ${ }^{*} P<.05$, Corrected for Multiple Comparisons)

\begin{tabular}{cccccc}
\hline & \multicolumn{2}{c}{ EDSS } & & \multicolumn{2}{c}{ T25FW } \\
\cline { 2 - 3 } \cline { 5 - 6 } MTR & $r$ & $P$ Value & & $R$ & $P$ Value \\
\hline Median whole brain & -.271 & $.003^{*}$ & & -.263 & $.004^{*}$ \\
Median NAWM & -.178 & .051 & & -.170 & .062 \\
Median WML & -.264 & $.003^{*}$ & -.328 & $<.001^{*}$ \\
Peak height whole brain & .033 & .719 & .022 & .807 \\
Peak height NAWM & -.042 & .646 & & -.118 & .196 \\
Peak height WML & -.101 & .273 & -.209 & $.021^{*}$ \\
MTsat & & & & \\
\hline Median whole brain & -.288 & $.001^{*}$ & -.225 & $.013^{*}$ \\
Median NAWM & -.238 & $.008^{*}$ & -.180 & $.048^{*}$ \\
Median WML & -.230 & $.011^{*}$ & -.251 & $.005^{*}$ \\
Peak height whole brain & .014 & .882 & .140 & .125 \\
Peak height NAWM & -.011 & .905 & -.004 & .964 \\
Peak height WML & -.017 & .852 & -.014 & .878 \\
\hline
\end{tabular}

EDSS $=$ Expanded Disability Status Scale; T25FW = timed 25-foot walk; MTR = magnetization transfer ratio; MTsat = magnetization transfer saturation; $\mathrm{NAWM}=$ brain normal appearing white matter; WML = brain white matter lesion volume.

found between median values in NAWM and both EDSS and T25FW. No significant disability correlations were found for median NAWM MTR.

Significant correlations were found between the median MTsat and EDSS for all cervical spinal cord regions (Table 3). No significant correlations were found between median MTR values and either the EDSS or T25FW.

Correlations between MTR and MTsat histogram metrics (median values, peak heights, and location) for the whole brain and either NAWM and the whole cervical spinal cord or the normal appearing spinal cord (NASC) were explored. No correlations were found between MTR or MTsat metrics obtained from the brain and the cervical cord although we found a trend toward a significant correlation (ie, those that reached $P<.017$, Bonferroni-corrected) between the median whole-brain MTsat and peak height of the whole cervical cord MTsat when controlling for age $(r=.348 ; P=.024)$.

Mixed model analyses revealed that median and peak height MTsat explained significant amounts of disability measures. In a model with the following variables: age, gender, and metrics (median and peak height) for the whole brain and c-spine, both the median whole-brain MTsat $(\beta=-.22[-.43 ;-.01], P=$ $.041)$ and peak height of the whole cervical spinal cord MTsat ( $\beta=-17.11$ [-32.84; -1.38$], P=.033$ ) showed significant associations with EDSS. The mixed model with age, gender, histogram metrics (median whole brain and c-spine), and region volumes (segmented lesions, brain, and cord) as independent variables showed significant associations for the median wholebrain MTsat $(\beta=-.30[-.59 ;-.01], P=.047)$ and median whole cervical spinal cord MTsat $(\beta=-.21[-.35 ;-.06], P=$ .006 ) with $\mathrm{T} 25 \mathrm{FW}$.

The mixed models did not provide evidence that wholebrain volume or individual (GM or WM) tissue volume fraction measures explained a significant proportion of the disability. Spearman rank-order correlations with EDSS were significant for T2 lesion volume $(r=-.254 ; P=.005)$, WM $(r=.181 ; P=$ $.048)$, and $\mathrm{GM}(r=-.189 ; P=.038)$ fractions.
Table 3. Spearman Rank-Order Correlation with Both Disability Scales for MTR and MTsat in the Cervical Spinal Cord ( $P<.05$, Corrected for Multiple Comparisons)

\begin{tabular}{cccccc}
\hline & \multicolumn{2}{c}{ EDSS } & & \multicolumn{2}{c}{ T25FW } \\
\cline { 2 - 3 } \cline { 5 - 6 } MTR & $r$ & $P$ Value & & $r$ & $P$ Value \\
\hline Median whole SC & -.228 & .104 & & -.055 & .700 \\
Median NASC & -.241 & .085 & & -.018 & .900 \\
Median SCL & -.380 & .109 & & -.275 & .254 \\
Peak height whole SC & -.465 & $.001^{*}$ & & -.190 & .176 \\
Peak height SCL & -.199 & .415 & & -.281 & .243 \\
MTsat & & & & \\
\hline Median whole SC & -.278 & $.046^{*}$ & -.094 & .506 \\
Median NASC & -.333 & $.016^{*}$ & -.128 & .365 \\
Median SCL & -.468 & $.043^{*}$ & -.378 & .110 \\
Peak height whole SC & -.377 & $.006^{*}$ & -.090 & .525 \\
Peak height SCL & -.014 & .954 & -.037 & .881 \\
\hline
\end{tabular}

EDSS = Expanded Disability Status Scale; T25FW = timed 25-foot walk MTR = magnetization transfer ratio; MTsat $=$ magnetization transfer saturation; $\mathrm{SC}=$ cervical spinal cord; NASC $=$ normal appearing spinal cord; $\mathrm{SCL}=$ cervical spinal cord lesions.

\section{Discussion}

Magnetization transfer provides a potentially useful complement to conventional MRI in assessment of patients with MS. ${ }^{23}$ Several studies have shown that MTR values in NAWM are altered in all of the major phenotypes of MS. Reduced MTR values have been found in NAWM, even in patients with clinically definite MS and very few or no T2 visible lesions. ${ }^{24}$ Quantitative magnetization transfer (qMT) allows parameters that reflect myelin integrity to be estimated independently of $\mathrm{T} 1$ and cross-relaxation rates between bound and unbound proton fractions. ${ }^{25}$ However, although qMT has a higher specificity for pathological processes underlying MS and is independent of MRI sequence, it requires long acquisition times and complex postprocessing, which have limited its application to animal models, postmortem, and experimental phase human imaging. ${ }^{26,27}$

MTR provides a measure reflecting microstructural information. ${ }^{15,20}$ In general, MS pathology is associated with a decrease in MTR: low MTR has been found in lesions that were not apparent on conventional MRI. Myelin loss (and, to a smaller extent, edema) reduces MTR values in $\mathrm{MS}^{21}$ Several studies have shown that MTR histogram-derived measures of the cervical cord correlate well with the clinical manifestations of MS and are related to demyelination. ${ }^{23,28}$ Our cohort had by definition a low to midlevel EDSS, with a maximum EDSS of 6.0. Other studies have included subjects with higher EDSS, which may explain our failure to identify a correlation between NAWM MTR and disability in this population.

Previous studies found that T2 lesion volume in the brain or spinal cord have not tended to show strong or significant correlations with the EDSS, $1,29,30$ although, in our population, there was a significant Spearman significant correlation of the brain T2 lesion volume, WM and GM fractions with EDSS. By contrast, we did not find simple correlation between cord volumes with EDSS and T25FW. Failure to identify some associations reported by others may reflect the restricted range of our sample of patients, who were mildly disabled (median EDSS $=3.5$ ), with a well-preserved ambulatory function and shorter disease duration (median disease duration $=2$ years). 
Studies that involved larger cohorts with a longer disease duration ( $>20$ years) and higher EDSS (median of 4, range $0-8$ ) have reported associations between upper spinal cord cross-sectional area and disability. ${ }^{31}$

No significant relationships between brain and cervical cord volumes and MTR/MTsat metrics were found and this suggests a nonuniform disease process in MS. The processes may affect each region independently or show differential involvement between patients. In the brain tissue, axonal transection has been documented at the center and the periphery of demyelinating lesions leading to axonal and tissue volume loss in the adjacent $\mathrm{WM}$, but in a large cross-sectional MRI study and in a postmortem study, no relationship between spinal cord atrophy and brain lesions was found. ${ }^{28,32,33}$

We hypothesized that MTsat measures, which take into account biophysical variations that may reduce the precision of MTR, may enhance the potential for discovery of clinicopathological correlations related to magnetization transfer. Consistent with this, we found significant correlations between median NAWM MTsat and EDSS and T25FW that were not found for MTR. Similar results were found for the cervical spinal cord, which also showed a correlation between median MTsat values and EDSS, although the same relationship was not found for MTR.

This apparent difference in sensitivity is likely to reflect differences between the two acquisition sequences to biophysical properties of tissue. A major contribution to decreased magnetization transfer in MS arises from reduced myelin volume, although increased tissue water also reduces MTR values in MS through effects on regional T $1{ }^{32}$ Lower axon density (which can vary between people with MS independently of differences in myelin density) also increases tissue $\mathrm{T} 1$ recovery time, and can itself decrease MTR $^{34}$ MTsat is relatively insensitive to T1-dependent effects, which are a significant source of variance in the MTR signal; this is likely to account for the greater contrast between tissue compartments seen with MTsat in the population observed here. The significantly lower correlation between MTsat and WML size versus MTR and lesion size that we observed this provides indirect evidence that MTsat is less dependent than MTR on lesion volumes, suggesting that it reflects microstructural damage outside of lesions to a greater extent.

Other studies are necessary to explore the limitations or advantages of MTsat versus conventional MT imaging in MS, preferably with a gold standard for comparison, such as histology from postmortem tissue. This could allow a direct determination of whether the MTsat technique was more sensitive to underlying axonal or cellular loss. Visual inspection of histograms noted that GM, WM, and CSF were much more separated in the MTsat versus MTR histograms. Similarly, the whole cervical spinal cord MTR histogram was unimodal, while that of MTsat was bimodal. A full comparison of the ability of MTR versus MTsat to discriminate tissue type needs to be performed.

The absence of a healthy control cohort is a major limitation of our study. Furthermore, no longitudinal data are available. Comparisons can only be made within the diseased group at a single timepoint. A full study tracking both healthy and MS subjects over time is the next logical step. The manual segmentations in the spinal cord were only performed by a single researcher due to the high labor required; no metrics of interreader reliability are available.
In conclusion, MTsat discriminated between disability levels in MS better than MTR in the NAWM and in the spinal cord in this study population of low to moderate EDSS and short disease duration. We speculate that MTsat may be more sensitive to microstructural tissue damage in MS than is MTR, and may prove useful and practicable for imaging in larger scale clinical studies and therapeutic trials.

\section{References}

1. Goodin DS. Magnetic resonance imaging as a surrogate outcome measure of disability in multiple sclerosis: have we been overly harsh in our assessment? Ann Neurol 2006;59(4):597-605.

2. Rovira A, Tintoré M. Spinal cord MRI should always be performed in clinically isolated syndrome patients: no. Mult Scler J $2014 ; 20(13): 1686-7$.

3. Tartaglino LM, Friedman DP, Flanders AE, et al. Multiple sclerosis in the spinal cord: MR appearance and correlation with clinical parameters. Radiology 1995;195(3):725-32.

4. Filippi M. Non-conventional MR techniques to monitor the evolution of multiple sclerosis. Neurol Sci 2001;22(2):195-200.

5. Barbosa S, Blumhardt LD, Roberts N, et al. Magnetic resonance relaxation time mapping in multiple sclerosis: normal appearing white matter and the "invisible" lesion load. Magn Reson Imaging $1994 ; 12(1): 33-42$.

6. DeLuca GC, Ebers GC, Esiri MM. Axonal loss in multiple sclerosis: a pathological survey of the corticospinal and sensory tracts. Brain 2004;127(5): 1009-18.

7. Evangelou N, DeLuca GC, Owens T, et al. Pathological study of spinal cord atrophy in multiple sclerosis suggests limited role of local lesions. Brain 2005; 128(Pt 1):29-34.

8. Gilmore CP, DeLuca GC, Bö L, et al. Spinal cord neuronal pathology in multiple sclerosis. Brain Pathol 2009;19(4):642-9.

9. Peterson JW, Bö L, Mörk S, et al. Transected neurites, apoptotic neurons, and reduced inflammation in cortical multiple sclerosis lesions. Ann Neurol 2001;50(3):389-400.

10. Vogt J, Paul F, Aktas O, et al. Lower motor neuron loss in multiple sclerosis and experimental autoimmune encephalomyelitis. Ann Neurol 2009;66(3):310-22.

11. Dousset V, Brochet B, Vital A, et al. Lysolecithin-induced demyelination in primates: preliminary in vivo study with MR and magnetization transfer. Am J Neuroradiol 1995;16(2):225-31.

12. Dousset V, Grossman RI, Ramer KN, et al. Experimental allergic encephalomyelitis and multiple sclerosis: lesion characterization with magnetization transfer imaging. Radiology 1992;182(2):48391.

13. Filippi M, Campi A, Dousset V, et al. A magnetization transfer imaging study of normal-appearing white matter in multiple sclerosis. Neurology 1995; $45(3 \mathrm{Pt} 1): 478-82$.

14. Tofts PS, Steens SCA, van Buchem MA. MT: magnetization transfer. In: Tofts P, ed. Quantitative MRI of the Brain. Chichester, UK: John Wiley \& Sons, Ltd, 2003:257-98.

15. Van Waesberghe JHTM, Kamphorst W, De Groot CJA, et al. Axonal loss in multiple sclerosis lesions: magnetic resonance imaging insights into substrates of disability. Ann Neurol 1999;46(5):747-54.

16. Helms G, Dathe H, Kallenberg K, Dechent P. High-resolution maps of magnetization transfer with inherent correction for RF inhomogeneity and T1 relaxation obtained from 3D FLASH MRI. Magn Reson Med 2008;60(6):1396-407.

17. Jack CR, Bernstein MA, Fox NC, et al. The Alzheimer's Disease Neuroimaging Initiative (ADNI): MRI methods. J Magn Reson Imaging 2008;27:685-91.

18. Helms G, Dathe H, Dechent P. Quantitative FLASH MRI at $3 T$ using a rational approximation of the Ernst equation. Magn Reson Med 2008;59(3):667-72.

19. Smith SM, Zhang Y, Jenkinson M, et al. Accurate, robust, and automated longitudinal and cross-sectional brain change analysis. Neuroimage 2002;17(1):479-89. 
20. van Buchem MA, McGowan JC, Kolson DL, et al. Quantitative volumetric magnetization transfer analysis in multiple sclerosis: estimation of macroscopic and microscopic disease burden. Magn Reson Med 1996;36(5):632-6.

21. Jenkinson M, Bannister P, Brady M, et al. Improved optimization for the robust and accurate linear registration and motion correction of brain images. Neuroimage 2002;17(2):825-41.

22. Diedenhofen B, Musch J. Cocor: a comprehensive solution for the statistical comparison of correlations. PLoS One 2015;10(4):e0121945.

23. Rovaris M, Filippi M. The value of new magnetic resonance tech niques in multiple sclerosis. Curr Opin Neurol 2000;13(3):249-54

24. De Stefano N, Narayanan S, Francis SJ, et al. Diffuse axonal and tissue injury in patients with multiple sclerosis with low cerebral lesion load and no disability. Arch Neurol 2002;59(10):1565-71

25. Morrison C, Henkelman RM. A model for magnetization transfer in tissues. Magn Reson Med 1995;33(4):475-82.

26. Schmierer K, Tozer DJ, Scaravilli F, et al. Quantitative magnetization transfer imaging in postmortem multiple sclerosis brain. $J$ Magn Reson Imaging 2007;26(1):41-51.

27. Dortch RD, Moore J, Li K, et al. Quantitative magnetization transfer imaging of human brain at 7T. Neuroimage 2013;64(1):640-9.
28. Lycklama A, Nijeholt GJ, Van Walderveen MAA, et al. Brain and spinal cord abnormalities in multiple sclerosis: correlation between MRI parameters, clinical subtypes and symptoms. Brain $1998 ; 121(4): 687-97$.

29. Gass A, Barker GJ, Kidd D, et al. Correlation of magnetization transfer ratio with clinical disability in multiple sclerosis. Ann Neurol 1994;36(1):62-7.

30. Barkhof $\mathrm{F}$. The clinico-radiological paradox in multiple sclerosis revisited. Curr Opin Neurol 2002;15(3):239-45.

31. Kearney H, Rocca MA, Valsasina P, et al. Magnetic resonance imaging correlates of physical disability in relapse onset multiple sclerosis of long disease duration. Mult Scler 2014;20(1): $72-80$.

32. Schmierer K, Scaravilli F, Altmann DR, et al. Magnetization transfer ratio and myelin in postmortem multiple sclerosis brain. Ann Neurol 2004;56(3):407-15.

33. Filippi M, Bozzali M, Horsfield MA, et al. A conventional and magnetization transfer MRI study of the cervical cord in patients with MS. Neurology 2000;54(1):207-13.

34. Bergers E, Bot JCJ, De Groot CJA, et al. Axonal damage in the spinal cord of MS patients occurs largely independent of T2 MRI lesions. Neurology 2002;59(11):1766-71. 


\section{APPENDIX C}

\section{Valorisation}

The work for this thesis is the outcome of a multidisciplinary research partnership between Maastricht University (Netherlands), GlaxoSmithKline plc (GSK) (United Kingdom), Imanova Ltd (United Kingdom) and the Marie Skłodowska-Curie Actions Innovative Training Networks (MSCA-ITN) (European Commission). The research was performed at the GSK Clinical Imaging Centre and Imanova facilities at the Hammersmith Hospital of Imperial College London with the aim of leveraging interactions between academia and industry in order to maximize the potential valorisation.

There is a long history of productive collaboration between biomedical researchers in academia and in the pharmaceutical industry. The primary beneficiary of this collaboration has been the public, who have benefited from the advances to healthcare and quality of life made possible, as well as the jobs creation and economic benefits arising from this strong sector of the European economy. Translational research interactions with academia have long contributed to the vibrancy of the pharmaceutical and related healthcare diagnostics industry. My research, jointly supervised by academics and industry mentors, provided an excellent opportunity to 
contribute to this and to derive considerable personal advantages for development of my capabilities.

The MSCA-ITN programme proved to be an ideal vehicle to overcome and cross boundaries in ways that promise to realise future applications of value for the healthcare, industry and the general public because of its emphasis on bringing stakeholders together for valorisation. In addition, the programme offered high level of mobility for the researcher involved and initiatives that support the process of strengthening knowledge and the experience. The international aspect of the programme made an important contribution to extending the scope of valorisation by helping me to better understand how to diffuse the ideas and work in multiple countries. The project implemented the ideas of Marie Skłodowska-Curie, who wrote: "I believe that international cooperation is a very difficult task, which has to be undertaken, should it even be at the cost of many efforts and true sacrifice"

Multiple sclerosis is an autoimmune disease of the central nervous system. It causes major neurological disability. Currently, there are more than 700,000 people in Europe, 100,000 people in the United Kingdom, 46,000 in Spain, 14,000 in Netherlands and more than 2 million people throughout the world with this disease. It is the most common disabling illness of young adults in the UK and it is twice as common in women as in men. Imaging plays a central role in the diagnosis and monitoring of the disease: the diagnosis made by the neurologists currently is based on the so called McDonald's criteria which allows diagnosis of multiple sclerosis on the basis of clinical findings and supported evidence from tests, the most important of which are magnetic resonance imaging of the brain and spinal cord. Magnetic resonance imaging is used not only to assess the diagnosis, but also to monitor the patients during their life. 
Conventional imaging has limitations in the evaluation of the causes of disability and lacks pathological specificity. My work has further explored an advanced method that promises greater sensitivity. Researchers, neurologists and neuropathologists can take advantage of new imaging techniques that can overcome those limitations. Among them is magnetization transfer, which can give quantitative information on the nature of pathological changes and add specificity to diagnostic images, thus enhancing assessment of lesion burden and the degree of disability.

Academia and industry have substantive differences in their approaches, but they share the common goal of improving the health of the patients. Industry relies on academia for basic research that identifies novel molecular targets, which in turn can be used in clinical trials that evaluate the efficacy and safety of derived interventions. Industry does not have the vast basic research laboratories and hospitals that exist in academia. Conversely, academia is reliant on industry for their discoveries to be translated into medicines and technologies used in healthcare. The discovery in the present thesis of potential brain and c-spine biomarkers from a novel quantitative MRI technique promises to allow future translation from academia to industry and healthcare applications. The present thesis invites for testing the proposed methodologies on larger groups of patients, after which further technical developments of developed technologies may become possible. In addition, the discovered biomarkers could be sensitive to other diseases of the central nervous system and could be used in other diagnostic studies, focusing perhaps on other regions of interest in the brain. In addition, the methods developed here could be useful in clinical trials for testing effects of existing and new immunosuppressive drugs. 
The implementation of the quantitative techniques performed in this thesis can be achieved in most of the European biomedical imaging research facilities and hospitals of the national health systems using stablished protocols. This provides a wide access to a broad established platform and an operational network where the derived biomarkers and parameters could contribute to the work of industry researchers and clinicians. It has highlighted a research and discovery space in new protocols could be developed to generalize the developed technology to ultra- high magnetic field technologies, as an attempt to overcome previous limitations, to further shed light to the genesis of the microstructural changes of the central nervous system tissues involved in neurodegenerative diseases and to create new opportunities for value realisation.

The pharmaceutical industry lives and breathes translational research. There are few organizations on the planet that can do what it has successfully accomplished repeatedly: transform knowledge of a potential drug target into a medicine for patients around the world. What the pharmaceutical industry does is often referred to as drug discovery using a wide bunch of techniques, among them, quantitative MRI. There are many opportunities for industry-academia collaborations using the big databases that are often collected by pharmaceutical companies (e.g., the GSK-Imperial College collaboration). These collaborations using 'big data' are expected to become increasingly important to achieve progress in the arena of the treatment of both neurodevelopmental and neurodegenerative diseases (such as multiple sclerosis). In this way, collaboration will spur innovation in the development of new medicines and in healthcare delivery. What my thesis work, embedded in an academic programme within industry, is that no single sector working alone can bring the needed medicines, delivery of care, and infrastructure to prevent and treat diseases of the 
world's population; collaboration between academia and the pharmaceutical industry is absolutely necessary and our results can be taken as the outcome of such an interaction. 


\section{Bibliography}

1. McDonald WI, Compston A, Edan G, Goodkin D, Hartung HP, Lublin FD, et al. Recommended diagnostic criteria for multiple sclerosis: Guidelines from the International Panel on the Diagnosis of Multiple Sclerosis. Ann Neurol. 2001;50(1):121-7.

2. Kurtzke JF. Rating neurologic impairment in multiple sclerosis: an expanded disability status scale (EDSS). Neurology. 1983;33(11):1444-52.

3. Fischer JS, Rudick R a, Cutter GR, Reingold SC. The Multiple Sclerosis Functional Composite Measure (MSFC): an integrated approach to MS clinical outcome assessment. National MS Society Clinical Outcomes Assessment Task Force. Mult Scler. 1999;5(4):244-50.

4. Medaer R. Does the history of multiple sclerosis go back as far as the 14th century? Acta Neurol Scand. 1979;60(3):189-92.

5. Charcot J. Histologie de la sclerose en plaques. Gaz Hop Civ Milit. 1868;41:554-8.

6. Charcot J. Lectures on diseases of the nervous system. Sigerson G, trans. 1877; Vol 1, Lon(The New Sydenham):158-222.

7. Ehrenberg C. Nothwendigkeit einer feineren mechanischen Zerlegung des Gehirns und der nerven von der chemischen, dargestellt aus beobachtungen von C. G. Ehrenberg. Annln Phys. 1833;28:449-73.

8. MCDONALD WI. THE EFFECTS OF EXPERIMENTAL DEMYELINATION ON CONDUCTION IN PERIPHERAL NERVE: A HISTOLOGICAL AND ELECTROPHYSIOLOGICAL STUDY. II. ELECTROPHYSIOLOGICAL OBSERVATIONS. Brain. ENGLAND; 1963 Sep;86:501-24.

9. McDonald WI, Sears TA. The effects of experimental demyelination on conduction in 
the central nervous system. Brain. ENGLAND; 1970;93(3):583-98.

10. Waxman SG. Conduction in myelinated, unmyelinated, and demyelinated fibers. Arch Neurol. 1977;34(10):585-9.

11. Prineas JW, Connell F. Remyelination in multiple sclerosis. Ann Neurol. UNITED STATES; 1979 Jan;5(1):22-31.

12. Rudick RA, Goodman A, Herndon RM, Panitch HS. Selecting relapsing remitting multiple sclerosis patients for treatment: the case for early treatment. J Neuroimmunol. NETHERLANDS; 1999 Jul;98(1):22-8.

13. Rudick R, Antel J, Confavreux C, Cutter G, Ellison G, Fischer J, et al. Recommendations from the National Multiple Sclerosis Society Clinical Outcomes Assessment Task Force. Ann Neurol. 1997;42(3):379-82.

14. Lechner-Scott J, Spencer B, de Malmanche T, Attia J, Fitzgerald M, Trojano M, et al. The frequency of CSF oligoclonal banding in multiple sclerosis increases with latitude. Mult Scler J. 2012;18(7):974-82.

15. Gronseth GS, Ashman EJ. Practice parameter: the usefulness of evoked potentials in identifying clinically silent lesions in patients with suspected multiple sclerosis (an evidence-based review): Report of the Quality Standards Subcommittee of the American Academy of Neurology. Neurology. UNITED STATES; 2000 May;54(9):1720-5.

16. Ebers GC, Bulman DE, Sadovnick AD, Paty DW, Warren S, Hader W, et al. A population-based study of multiple sclerosis in twins. $\mathrm{N}$ Engl $\mathrm{J}$ Med. 1986;315(26):1638-42.

17. Ebers GC, Sadovnick AD, Risch NJ. A genetic basis for familial aggregation in multiple sclerosis. Canadian Collaborative Study Group. Nature. 1995. p. 150-1.

18. Oksenberg JR, Baranzini SE, Sawcer S, Hauser SL. The genetics of multiple sclerosis: 
SNPs to pathways to pathogenesis. Nat Rev Genet. 2008;9(7):516-26.

19. Noseworthy JH. Progress in determining the causes and treatment of multiple sclerosis. Nature. 1999;399(6738 Suppl):A40-7.

20. Sadovnick AD, Ebers GC. Epidemiology of multiple sclerosis: a critical overview. Can J Neurol Sci. 1993;20(1):17-29.

21. Fainardi E, Castellazzi M, Seraceni S, Granieri E, Contini C. Under the microscope: focus on Chlamydia pneumoniae infection and multiple sclerosis. Curr Neurovasc Res. 2008;5(1):60-70.

22. Hayes CE, Donald Acheson E. A unifying multiple sclerosis etiology linking virus infection, sunlight, and vitamin D, through viral interleukin-10. Med Hypotheses. 2008;71(1):85-90.

23. Willis SN, Stadelmann C, Rodig SJ, Caron T, Gattenloehner S, Mallozzi SS, et al. Epstein-Barr virus infection is not a characteristic feature of multiple sclerosis brain. Brain. 2009;132(12):3318-28.

24. Geurts JJG, Calabrese M, Fisher E, Rudick RA. Measurement and clinical effect of grey matter pathology in multiple sclerosis. Lancet Neurol. England; 2012 Dec;11(12):1082-92.

25. Charil A, Dagher A, Lerch JP, Zijdenbos AP, Worsley KJ, Evans AC. Focal cortical atrophy in multiple sclerosis: Relation to lesion load and disability. Neuroimage. 2007;34(2):509-17.

26. Reynolds R, Roncaroli F, Nicholas R, Radotra B, Gveric D, Howell O. The neuropathological basis of clinical progression in multiple sclerosis. Acta Neuropathologica. 2011. p. 155-70.

27. Peterson JW, B?? L, M??rk S, Chang A, Trapp BD, Bö L, et al. Transected neurites, apoptotic neurons, and reduced inflammation in cortical multiple sclerosis lesions. Ann 
Neurol. 2001;50(3):389-400.

28. Miller DH, Rudge P, Johnson G, Kendall BE, Macmanus DG, Moseley IF, et al. Serial gadolinium enhanced magnetic resonance imaging in multiple sclerosis. Brain. 1988;111 ( Pt 4(Pt 4):927-39.

29. Kutzelnigg A, Lucchinetti CF, Stadelmann C, Brück W, Rauschka H, Bergmann M, et al. Cortical demyelination and diffuse white matter injury in multiple sclerosis. Brain. 2005;128(11):2705-12.

30. Frohman TC, Davis SL, Beh S, Greenberg BM, Remington G, Frohman EM. Uhthoff's phenomena in MS--clinical features and pathophysiology. Nat Rev Neurol. 2013;9(9):535-40.

31. Sears TA, Bostock H. Conduction failure in demyelination: is it inevitable? Adv Neurol. UNITED STATES; 1981;31:357-75.

32. Lucchinettil CF, Bruckz W, Rodriguez ' M, Lassmann3 H, Lassmann H. Distinct Patterns of Multiple Sclerosis Pathology Indicates Heterogeneity in Pathogenesis. Brain Pathol. 1996;6:259-74.

33. Losseff NA, Wang L, Lai HM, Yoo DS, Gawne-Cain ML, McDonald WI, et al. Progressive cerebral atrophy in multiple sclerosis. A serial MRI study. Brain. 1996;119(6):2009-19.

34. Naruse S, Horikawa Y, Tanaka C, Hirakawa K, Nishikawa H, Yoshizaki K. Proton nuclear magnetic resonance studies on brain edema. J Neurosurg. 1982;56(6):747-52.

35. Filippi M, Campi a, Dousset V, Baratti C, Martinelli V, Canal N, et al. A magnetization transfer imaging study of normal-appearing white matter in multiple sclerosis. Neurology. 1995;45(3 Pt 1):478-82.

36. Edzes HT, Samulski ET. The measurement of cross-relaxation effects in the proton NMR spin-lattice relaxation of water in biological systems: Hydrated collagen and 
muscle. J Magn Reson. Academic Press; 1978 Aug;31(2):207-29.

37. Matthews PM, Pioro E, Narayanan S, De Stefano N, Fu L, Francis G, et al. Assessment of lesion pathology in multiple sclerosis using quantitative MRI morphometry and magnetic resonance spectroscopy. Brain. 1996;119 ( Pt 3:715-22.

38. BROWNELL B, HUGHES JT. The distribution of plaques in the cerebrum in multiple sclerosis. J Neurol Neurosurg Psychiatry. Not Available; 1962 Nov;25:315-20.

39. Lumsden C. The neuropathology of multiple sclerosis. Handbook o. Amsterdam: Elsevier Science Publishers; 1970. 217-309 p.

40. Bozzali M, Cercignani M, Sormani MP, Comi G, Filippi M. Quantification of brain gray matter damage in different MS phenotypes by use of diffusion tensor MR imaging. Am J Neuroradiol. 2002;23(6):985-8.

41. Bakshi R, Benedict RHB, Bermel RA, Caruthers SD, Puli SR, Tjoa CW, et al. T2 hypointensity in the deep gray matter of patients with multiple sclerosis: a quantitative magnetic resonance imaging study. Arch Neurol. 2002;59(1):62-8.

42. Grimaud J, Millar J, Thorpe JW, Moseley IF, McDonald WI, Miller DH. Signal intensity on MRI of basal ganglia in multiple sclerosis. J Neurol Neurosurg Psychiatry. 1995;59(3):306-8.

43. Moraal B, Roosendaal SD, Pouwels PJW, Vrenken H, van Schijndel R a, Meier DS, et al. Multi-contrast, isotropic, single-slab 3D MR imaging in multiple sclerosis. Eur Radiol. 2008;18(10):2311-20.

44. ARKIN H, SHERMAN IC, WEINBERG SL. Tetraethylammonium chloride in the treatment of multiple sclerosis. AMA Arch Neurol Psychiatry. Not Available; 1950 Oct;64(4):536-45.

45. ALEXANDER L. New concept of critical steps in course of chronic debilitating neurologic disease in evaluation of therapeutic response; a longitudinal study of 
multiple sclerosis by quantitative evaluation of neurologic involvement and disability. AMA Arch Neurol Psychiatry. Not Available; 1951 Sep;66(3):253-71.

46. KURTZKE JF. A new scale for evaluating disability in multiple sclerosis. Neurology. Not Available; 1955 Aug;5(8):580-3.

47. Rosti-Otajärvi E, Hämäläinen P, Koivisto K, Hokkanen L. The reliability of the MSFC and its components. Acta Neurol Scand. 2008;117(6):421-7.

48. Hobart J, Blight AR, Goodman A, Lynn F, Putzki N. Timed 25-Foot Walk: Direct evidence that improving $20 \%$ or greater is clinically meaningful in MS. Neurology. 2013;80(16):1509-17.

49. Goldman MD, Motl RW, Scagnelli J, Pula JH, Sosnoff JJ, Cadavid D. Clinically meaningful performance benchmarks in MS:Timed 25-Foot Walk and the real world. Neurology. 2013;81(21):1856-63.

50. Lauterbur PC. Image Formation by Induced Local Interactions: Examples Employing Nuclear Magnetic Resonance. Nature. 1973;242(5394):190-1.

51. Damadian R, Goldsmith M, Minkoff L. NMR in cancer: XVI. FONAR image of the live human body. Physiological chemistry and physics. 1977. p. 97.

52. Hinshaw WS, Bottomley PA, Holland GN. Radiographic thin-section image of the human wrist by nuclear magnetic resonance. Nature. 1977;270(5639):722-3.

53. Rydberg JN, Hammond CA, Grimm RC, Erickson BJ, Jack CR, Huston J, et al. Initial Clinical-Experience in Mr-Imaging of the Brain with a Fast Fluid-Attenuated Inversion-Recovery Pulse Sequence. Radiology. 1994;193(1):173-80.

54. Bailes DR, Young IR, Thomas DJ, Straughan K, Bydder GM, Steiner RE. NMR imaging of the brain using spin-echo sequences. Clin Radiol. ENGLAND; 1982 Jul;33(4):395-414.

55. Van Waesberghe JHTM, Kamphorst W, De Groot CJ a, Van Walderveen MAA, 
Castelijns J a., Ravid R, et al. Axonal loss in multiple sclerosis lesions: Magnetic resonance imaging insights into substrates of disability. Ann Neurol. 1999;46(5):74754.

56. Larsson H, Frederiksen J. Assessment of demyelination, edema, and gliosis by in vivo determination of $\mathrm{T} 1$ and $\mathrm{T} 2$ in the brain of patients with acute attack of multiple sclerosis. Magn Reson Med. 1989;348:337-48.

57. Larsson HB, Frederiksen J, Kjaer L, Henriksen O, Olesen J. In vivo determination of $\mathrm{T} 1$ and $\mathrm{T} 2$ in the brain of patients with severe but stable multiple sclerosis. Magn Reson Med. 1988;7(1):43-55.

58. Molyneux PD, Barker GJ, Barkhof F, Beckmann K, Dahlke F, Filippi M, et al. Clinical-MRI correlations in a European trial of interferon beta-1b in secondary progressive MS. Neurology. 2001;57(12):2191-7.

59. Simon JH, Jacobs LD, Campion M, Wende K, Simonian N, Cookfair DL, et al. Magnetic resonance studies of intramuscular interferon beta-1a for relapsing multiple sclerosis. The Multiple Sclerosis Collaborative Research Group. Ann Neurol. UNITED STATES; 1998 Jan;43(1):79-87.

60. Forsén S, Hoffman R, Forsén S, Hoffman R, Forsén S, Hoffman R. Study of moderately rapid chemical exchange reactions by means of nuclear magnetic double resonance. J Chem Phys. 2004;39(11):2892-901.

61. Edzes, HT Samulski E. Cross relaxation and spin diffusion in proton NMR of hydrated collagen. Nature. 1977;165:521-3.

62. Wolff SD, Balaban RS. Magnetization transfer contrast (MTC) and tissue water proton relaxation in vivo. Magn Reson Med. 1989;10(1):135-44.

63. Harrison R, Bronskill MJ, Henkelman RM. Magnetization transfer and T2 relaxation components in tissue. Magn Reson Med. 1995;33(4):490-6. 
64. Morris GA, Freemont AJ. Direct observation of the magnetization exchange dynamics responsible for magnetization transfer contrast in human cartilage in vitro. Magn Reson Med. 1992;28(1):97-104.

65. Balaban RS, Ceckler TL. Magnetization transfer contrast in magnetic resonance imaging. Magn Reson Q. 1992;8(2):116-37.

66. Eng J, Ceckler TL, Balaban RS. Quantitative $1 \mathrm{H}$ magnetization transfer imaging in vivo. Magn Reson Med. 1991;17(2):304-14.

67. Dixon W. Use of magnetization transfer pulse sequences for clinical use. J Comput Assist Tomogr. 1992;16:7-18.

68. Ordidge RJ, Knight RA, Helpern JA. Magnetization transfer contrast (MTC) in FLASH MR imaging. Magn Reson Imaging. 1991;9(6):889-93.

69. Dousset V, Grossman RI, Ramer KN, Schnall MD, Young LH, Gonzalez-Scarano F, et al. Experimental allergic encephalomyelitis and multiple sclerosis: lesion characterization with magnetization transfer imaging. Radiology. 1992;182(2):483-91.

70. Frahm J, Haase A, Matthaei D. Rapid NMR imaging of dynamic processes using the FLASH technique. Magn Reson Med. 1986;3(2):321-7.

71. Helms G, Dathe H, Kallenberg K, Dechent P. High-resolution maps of magnetization transfer with inherent correction for RF inhomogeneity and T1 relaxation obtained from 3D FLASH MRI. Magn Reson Med. 2008;60(6):1396-407.

72. Filippi M, Horsfield MA, Tofts PS, Barkhof F, Thompson AJ, Miller DH. Quantitative assessment of $\{\mathrm{MRI}\}$ lesion load in monitoring the evolution of multiple sclerosis. Brain. 1995;118(6):1601-12.

73. Filippi M, Horsfield MA, Ad??r HJ, Barkhof F, Bruzzi P, Evans A, et al. Guidelines for using quantitative measures of brain magnetic resonance imaging abnormalities in monitoring the treatment of multiple sclerosis. Ann Neurol. 1998;43(4):499-506. 
74. Rovaris M, Filippi M. Magnetic resonance techniques to monitor disease evolution and treatment trial outcomes in multiple sclerosis. Curr Opin Neurol. 1999;12(3):337-44.

75. Gawne-Cain ML, O’Riordan JI, Coles A, Newell B, Thompson AJ, Miller DH. MRI lesion volume measurement in multiple sclerosis and its correlation with disability: a comparison of fast fluid attenuated inversion recovery (fFLAIR) and spin echo sequences. J Neurol Neurosurg Psychiatry. 1998;64(2):197-203.

76. Hiehle JF, Grossman RI, Ramer KN, Gonzalez-Scarano F, Cohen JA. Magnetization transfer effects in MR-detected multiple sclerosis lesions: Comparison with gadolinium-enhanced spin-echo images and nonenhanced T1- weighted images. Am J Neuroradiol. 1995;16(1):69-77.

77. Petrella JR, Grossman RI, McGowan JC, Campbell G, Cohen JA. Multiple sclerosis lesions: Relationship between MR enhancement pattern and magnetization transfer effect. Am J Neuroradiol. 1996;17(6):1041-9.

78. Schmierer K, Scaravilli F, Altmann DR, Barker GJ, Miller DH. Magnetization transfer ratio and myelin in postmortem multiple sclerosis brain. Ann Neurol. 2004;56(3):40715.

79. Van Waesberghe JHTM, Van Walderveen MAA, Castelijns JA, Scheltens P, Lycklama ?? Nijeholt GJ, Polman CH, et al. Patterns of lesion development in multiple sclerosis: Longitudinal observations with T1-weighted spin-echo and magnetization transfer MR. In: American Journal of Neuroradiology. 1998. p. 675-83.

80. Van Waesberghe JHTM, Castelijns JA, Scheltens P, Truyen L, Lycklama ?? Nijeholt GJ, Hoogenraad FG, et al. Comparison of four potential MR parameters for severe tissue destruction in multiple sclerosis lesions. Magn Reson Imaging. 1997;15(2):15562.

81. Guo AC, Jewells VL, Provenzale JM. Analysis of normal-appearing white Matter in 
multiple sclerosis: Comparison of diffusion tensor MR imaging and magnetization transfer imaging. Am J Neuroradiol. 2001;22(10):1893-900.

82. Loevner LA, Grossman RI, Cohen JA, Lexa FJ, Kessler D, Kolson DL. Microscopic disease in normal-appearing white matter on conventional MR images in patients with multiple sclerosis: assessment with magnetization-transfer measurements. Radiology. 1995

83. De Stefano N, Narayanan S, Francis SJ, Smith S, Mortilla M, Tartaglia MC, et al. Diffuse axonal and tissue injury in patients with multiple sclerosis with low cerebral lesion load and no disability. Arch Neurol. 2002;59(10):1565-71.

84. Filippi M, Rocca MA, Minicucci L, Martinelli V, Ghezzi A, Bergamaschi R, et al. Magnetization transfer imaging of patients with definite MS and negative conventional MRI. Neurology. 1999;52(4):845-8.

85. Tortorella C, Viti B, Bozzali M, Sormani MP, Rizzo G, Gilardi MF, et al. A magnetization transfer histogram study of normal-appearing brain tissue in MS. Neurology. 2000;54(1):186-93.

86. Hickman SJ, Hadjiprocopis A, Coulon O, Miller DH, Barker GJ. Cervical spinal cord MTR histogram analysis in multiple sclerosis using a 3D acquisition and a B-spline active surface segmentation technique. Magn Reson Imaging. 2004;22(6):891-5.

87. Rovaris M, Gallo a, Riva R, Ghezzi a, Bozzali M, Benedetti B, et al. An MT MRI study of the cervical cord in clinically isolated syndromes suggestive of MS. Neurology. 2004;63:584-5.

88. Levesque I, Sled JG, Narayanan S, Santos AC, Brass SD, Francis SJ, et al. The role of edema and demyelination in chronic T1 black holes: A quantitative magnetization transfer study. J Magn Reson Imaging. 2005;21(2):103-10.

89. Helms G, Dathe H, Dechent P. Quantitative FLASH MRI at 3T using a rational 
approximation of the Ernst equation. Magn Reson Med. 2008;59(3):667-72.

90. van Buchem M a, McGowan JC, Kolson DL, Polansky M, Grossman RI. Quantitative volumetric magnetization transfer analysis in multiple sclerosis: estimation of macroscopic and microscopic disease burden. Magn Reson Med. 1996;36(5):632-6.

91. Polman CH, Reingold SC, Banwell B, Clanet M, Cohen JA, Filippi M, et al. Diagnostic criteria for multiple sclerosis: 2010 Revisions to the McDonald criteria. Ann Neurol. 2011;69(2):292-302.

92. Altman DG, Bland JM. Measurement in Medicine: the Analysis of Method Comparison Studies. Statistician. 1983;32(July 1981):307-17.

93. Bland JM, Altman DG. Statistical Methods for Assessing Agreement Between Two Methods of Clinical Measurement. Lancet. 1986;327:307-10.

94. Rovaris M, Filippi M. The value of new magnetic resonance techniques in multiple sclerosis. CurrOpinNeurol. 2000;13(3):249-54.

95. Barbosa S, Blumhardt LD, Roberts N, Lock T, Edwards RHTHT. Magnetic resonance relaxation time mapping in multiple sclerosis: Normal appearing white matter and the “invisible” lesion load. Magn Reson Imaging. 1994;12(1):33-42.

96. Gass A, Barker GJ, Kidd D, Thorpe JW, MacManus D, Brennan A, et al. Correlation of magnetization transfer ratio with clinical disability in multiple sclerosis. Ann Neurol. 1994;36(1):62-7.

97. Zhou LQ, Zhu YM, Grimaud J, Hermier M, Rovaris M, Filippi M. A new method for analyzing histograms of brain magnetization transfer ratios: Comparison with existing techniques. Am J Neuroradiol. 2004;25(7):1234-41.

98. Filippi M, Rovaris M. Magnetisation transfer imaging in multiple sclerosis. J Neurovirol. 2000;6 Suppl 2:S115-20.

99. Filippi M, Iannucci G, Tortorella C, Minicucci L, Horsfield MA, Colombo B, et al. 
Comparison of MS clinical phenotypes using conventional and magnetization transfer MRI. Neurology. 1999;52(3):588-94.

100. De Stefano N, Narayanan S, Francis GS, Arnaoutelis R, Tartaglia MC, Antel JP, et al. Evidence of axonal damage in the early stages of multiple sclerosis and its relevance to disability. Arch Neurol. 2001;58(1):65-70.

101. Filippi M, Agosta F. Magnetization transfer MRI in multiple sclerosis. Journal of Neuroimaging. 2007.

102. Ge Y, Grossman RI, Udupa JK, Babb JS, Kolson DL, McGowan JC. Magnetization transfer ratio histogram analysis of gray matter in relapsing-remitting multiple sclerosis. AJNR Am J Neuroradiol. 2001;22(3):470-5.

103. Ge Y, Grossman RI, Babb JS, He J, Mannon LJ. Dirty-Appearing White Matter in Multiple Sclerosis: Volumetric MR Imaging and Magnetization Transfer Ratio Histogram Analysis. Am J Neuroradiol. 2003;24(10):1935-40.

104. Bjartmar C, Kidd G, M??rk S, Rudick R, Trapp BD. Neurological disability correlates with spinal cord axonal loss and reduced $\mathrm{N}$-acetyl aspartate in chronic multiple sclerosis patients. Ann Neurol. 2000;48(6):893-901.

105. Lycklama AN, Uitdehaag BMJ, Bergers E, Castelijns JA, Polman CH, Barkhof F. Spinal cord magnetic resonance imaging in suspected multiple sclerosis. Eur Radiol. 2000;10(2):368-76.

106. Allen I V, McQuaid S, Mirakhur M, Nevin G. Pathological abnormalities in the normal-appearing white matter in multiple sclerosis. Neurol Sci. 2001;22(2):141-4.

107. Allen I V., McKeown SR. A histological, histochemical and biochemical study of the macroscopically normal white matter in multiple sclerosis. J Neurol Sci. 1979;41(1):81-91.

108. Rovaris M, Bozzali M, Santuccio G, Iannucci G, Sormani MP, Colombo B, et al. 
Relative contributions of brain and cervical cord pathology to multiple sclerosis disability: a study with magnetisation transfer ratio histogram analysis. J Neurol Neurosurg Psychiatry. 2000;69(6):723-7.

109. Oh J, Saidha S, Chen M, Smith SA, Prince J, Jones C, et al. Spinal cord quantitative MRI discriminates between disability levels in multiple sclerosis. Neurology. 2013;80(6):540-7.

110. Barkhof F. The clinico-radiological paradox in multiple sclerosis revisited. Curr Opin Neurol. 2002;15(3):239-45.

111. Filippi M, Grossman RI. MRI techniques to monitor MS evolution: the present and the future. Neurology. 2002;58:1147-53.

112. Filippi M, Bozzali M, Horsfield M a, Rocca M a, Sormani MP, Iannucci G, et al. A conventional and magnetization transfer MRI study of the cervical cord in patients with MS. Neurology. 2000;54(1):207-13.

113. Lycklama ?? Nijeholt GJ, Van Walderveen MAA, Castelijns J a., Van Waesberghe JHTM, Polman C, Scheltens P, et al. Brain and spinal cord abnormalities in multiple sclerosis: Correlation between MRI parameters, clinical subtypes and symptoms. Brain. 1998;121(4):687-97.

114. Bergers E, Bot JCJ, De Groot CJ a, Polman CH, Lycklama à Nijeholt GJ, Castelijns J a, et al. Axonal damage in the spinal cord of MS patients occurs largely independent of T2 MRI lesions. Neurology. 2002;59(11):1766-71.

115. DeLuca GC, Ebers GC, Esiri MM. Axonal loss in multiple sclerosis: A pathological survey of the corticospinal and sensory tracts. Brain. 2004;127(5):1009-18.

116. Evangelou N, DeLuca GC, Owens T, Esiri MM. Pathological study of spinal cord atrophy in multiple sclerosis suggests limited role of local lesions. Brain. 2005;128(Pt 1):29-34. 
117. Gilmore CP, DeLuca GC, Bö L, Owens T, Lowe J, Esiri MM, et al. Spinal cord neuronal pathology in multiple sclerosis. Brain Pathol. 2009;19(4):642-9.

118. Vogt J, Paul F, Aktas O, M??ller-Wielsch K, D??rr J, D??rr S, et al. Lower motor neuron loss in multiple sclerosis and experimental autoimmune encephalomyelitis. Ann Neurol. 2009;66(3):310-22.

119. Ormerod IE, Miller DH, McDonald WI, du Boulay EP, Rudge P, Kendall BE, et al. The role of NMR imaging in the assessment of multiple sclerosis and isolated neurological lesions. A quantitative study. Brain. 1987;110 ( Pt 6:1579-616.

120. Kidd D, Thorpe JW, Thompson AJ, Kendall BE, Moseley IF, MacManus DG, et al. Spinal cord MRI using multi-array coils and fast spin echo. II. Findings in multiple sclerosis. Neurology. 1993;43(12):2632263-7.

121. Tartaglino LM, Friedman DP, Flanders AE, Lublin FD, Knobler RL, Liem M. Multiple sclerosis in the spinal cord: MR appearance and correlation with clinical parameters. Radiology. 1995;195(3):725-32.

122. Filippi M. The role of non-conventional magnetic resonance techniques in monitoring evolution of multiple sclerosis. J Neurol Neurosurg Psychiatry. 1998;64 Suppl 1:S528.

123. Dousset V, Brochet B, Vital A, Gross C, Benazzouz A, Boullerne A, et al. Lysolecithin-induced demyelination in primates: Preliminary in vivo study with MR and magnetization transfer. Am J Neuroradiol. 1995;16(2):225-31.

124. Tofts PS, Steens SC a, van Buchem MA. MT: Magnetization Transfer. Quantitative MRI of the Brain. 2003. 257-298 p.

125. Stankiewicz JM, Neema M, Alsop DC, Healy BC, Arora A, Buckle GJ, et al. Spinal cord lesions and clinical status in multiple sclerosis: A $1.5 \mathrm{~T}$ and $3 \mathrm{~T}$ MRI study. J Neurol Sci. 2009;279(1-2):99-105. 
126. Zivadinov R, Leist TP. Clinical-magnetic resonance imaging correlations in multiple sclerosis. J Neuroimaging. 2005;15(4 Suppl):10S - 21S.

127. Kearney H, Rocca M a, Valsasina P, Balk L, Sastre-Garriga J, Reinhardt J, et al. Magnetic resonance imaging correlates of physical disability in relapse onset multiple sclerosis of long disease duration. Mult Scler. 2014;20(1):72-80.

128. Ferguson B, Matyszak MK, Esiri MM, Perry VH. Axonal damage in acute multiple sclerosis lesions. Brain. 1997;120 ( Pt 3(3):393-9.

129. Ganter P, Prince C, Esiri MM. Spinal cord axonal loss in multiple sclerosis: a postmortem study. Neuropathol Appl Neurobiol. 1999;25(6):459-67.

130. Klein JP, Arora a., Neema M, Healy BC, Tauhid S, Goldberg-Zimring D, et al. A 3T MR imaging investigation of the topography of whole spinal cord atrophy in multiple sclerosis. Am J Neuroradiol. 2011;32(6):1138-42.

131. O'Riordan JI, Losseff N a, Phatouros C, Thompson a J, Moseley IF, MacManus DG, et al. Asymptomatic spinal cord lesions in clinically isolated optic nerve, brain stem, and spinal cord syndromes suggestive of demyelination. J Neurol Neurosurg Psychiatry. 1998;64(3):353-7.

132. Ropele S, Seifert T, Enzinger C, Fazekas F. Method for quantitative imaging of the macromolecular 1H fraction in tissues. Magn Reson Med. 2003;49(5):864-71.

133. Graham SJ, Henkelman RM. Understanding pulsed magnetization transfer. J Magn Reson Imaging. 1997;7(5):903-12.

134. Henkelman RM, Huang X, Xiang QS, Stanisz GJ, Swanson SD, Bronskill MJ. Quantitative interpretation of magnetization transfer. Magn Reson Med. 1993;29(6):759-66.

135. Pike GB, De Stefano N, Narayanan S, Worsley KJ, Pelletier D, Francis GS, et al. Multiple sclerosis: magnetization transfer MR imaging of white matter before lesion 
appearance on T2-weighted images. Radiology. 2000;215:824-30. 